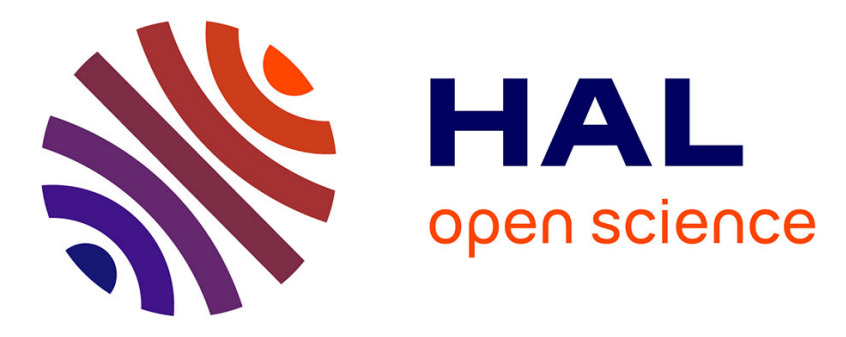

\title{
Unusual One-Armed Density Waves in the Cassini Division of Saturn's Rings
}

\author{
Richard G French, Colleen A Mcghee-French, Philip Nicholson, Mathew M \\ Hedman, Nicole J Rappaport, Essam A Marouf, Pierre-Yves Longaretti, Joe
} Hahn

\section{To cite this version:}

Richard G French, Colleen A Mcghee-French, Philip Nicholson, Mathew M Hedman, Nicole J Rappaport, et al.. Unusual One-Armed Density Waves in the Cassini Division of Saturn's Rings. Icarus, 2020, 339, pp.113600. 10.1016/j.icarus.2019.113600 . hal-02404628

\section{HAL Id: hal-02404628 \\ https://hal.science/hal-02404628}

Submitted on 11 Dec 2019

HAL is a multi-disciplinary open access archive for the deposit and dissemination of scientific research documents, whether they are published or not. The documents may come from teaching and research institutions in France or abroad, or from public or private research centers.
L'archive ouverte pluridisciplinaire HAL, est destinée au dépôt et à la diffusion de documents scientifiques de niveau recherche, publiés ou non, émanant des établissements d'enseignement et de recherche français ou étrangers, des laboratoires publics ou privés. 
Draft VERsion November 29, 2019

Typeset using $\mathrm{LAT}_{\mathrm{E}} \mathrm{X}$ modern style in AASTeX63

\title{
Unusual One-Armed Density Waves in the Cassini Division of Saturn's Rings
}

\author{
Richard G. French, Colleen A. McGhee-French, ${ }^{1}$ Philip D. Nicholson ${ }^{2}$ \\ Mathew M. Hedman,${ }^{3}$ Nicole J. Rappaport, ${ }^{4}$ Essam A. Marouf,${ }^{5}$ \\ Pierre-Yves Longaretti ${ }^{6}$ AND Joe $\mathrm{HahN}^{7}$ \\ ${ }^{1}$ Department of Astronomy, Wellesley College, Wellesley MA 02481 \\ ${ }^{2}$ Department of Astronomy, Cornell University, Ithaca NY 14853 \\ ${ }^{3}$ Department of Physics, University of Idaho, Moscow, ID 83844 \\ ${ }^{4}$ Cassini Radio Science Team, Jet Propulsion Laboratory (retired), Pasadena, CA 91109 \\ ${ }^{5}$ San Jose State University, San Jose, CA 95192 \\ ${ }^{6}$ Institute of Planetology and Astrophysics of Grenoble, France \\ ${ }^{7}$ Space Science Institute, Cedar Park, TX 78613
}

\begin{abstract}
We identify several unusual one-armed density waves in Saturn's Cassini Division, using occultation observations from the Cassini RSS and VIMS instruments. In the inner Cassini Division, we identify outward-propagating density waves with wavenumber $m=1$ located near 118,050 km (W118.05), 118,400 km (W118.40), and 118,530 km (W118.53). From Cassini ISS images taken at very low incidence angles we find evidence for vertical structure in these waves, which may be evidence for splashing of ring material in the crests of the waves. We propose that these waves are driven by nearby eccentric ringlets, probably the Strange (or R6) and Herschel ringlets. In the outer Cassini Division, we identify a wave structure near 120,200 km (W120.20), between the Laplace and Bessel gaps, as a conspicuous example of a standing wave in a planetary ring system. It appears to be the superposition of an outward-propagating $m=1$ density wave, probably driven by the nearby Laplace ringlet, and its reflection at the inner edge of the Bessel gap.
\end{abstract}

Keywords: occultations, planets: rings

\section{INTRODUCTION}

Corresponding author: Richard G. French

rfrench@wellesley.edu 
Over a decade of Cassini stellar and radio occultation experiments have provided ample data for investigations of organized, km-scale structure in Saturn's rings in ways that were previously impossible. This work has included studies of satellite-driven density and bending waves in the $\mathrm{C}$ ring, $\mathrm{B}$ ring and Cassini Division (Colwell et al. 2009a; Baillié et al. 2011; Nicholson and Hedman 2016; Hedman and Nicholson 2016); non-circular ringlets and gap edges (Nicholson et al. 2014a,b; French et al. 2016a), many of which are perturbed by satellite resonances or free normal modes; and waves in the $\mathrm{C}$ ring driven by internal oscillations in Saturn or by quasi-permanent gravity anomalies within the planet (Hedman and Nicholson 2013, 2014; French et al. 2016b, 2019; Hedman et al. 2019). In this same period, studies of large sequences of Cassini imaging data have made it possible to characterize the non-circular outer edges of the A and B rings (Spitale and Porco 2009, 2010; El Moutamid et al. 2016), as well as the perturbed edges of the Encke and Keeler gaps in the outer A ring (Tiscareno et al. 2005; Weiss et al. 2009; Tajeddine et al. 2017) and a large number of satellite-driven density and bending waves, chiefly in the A ring (Tiscareno and Harris 2018).

The Cassini Division, located between the bright, optically thick A and B rings, is one of the most dynamically complex regions of Saturn's rings. With an average normal optical depth $\tau \simeq 0.1$ and a relatively low single particle albedo $\varpi_{0} \simeq 0.25$ (Cooke 1991), it more closely resembles the $\mathrm{C}$ ring than either the $\mathrm{A}$ or $\mathrm{B}$ rings (Cuzzi et al. 1984; Esposito et al. 1984). Overviews of the Cassini Division and its salient features are shown in a Cassini image in Fig. 1 and in a radial occultation profile in Fig. 2. Although only $4500 \mathrm{~km}$ wide, this region is host to no fewer than eight narrow gaps (Colwell et al. 2009b). Its inner edge is defined by the outer edge of the B ring, long known to be perturbed by the 2:1 Inner Lindblad Resonance (ILR) with the satellite Mimas (Goldreich and Tremaine 1978; Porco et al. 1984) but now recognized also to be the site of several additional normal modes with azimuthal wavenumbers ranging from $m=1$ to $m=5$ and radial amplitudes between 5.6 and 37.0 km (Spitale and Porco 2010; Nicholson et al. 2014a).

Initial studies of other gap edges in this region using stellar occultation data from the Visual and Infrared Mapping Spectrometer (VIMS) instrument on Cassini as well as radio occultations carried out with the spacecraft's Radio Science Subsystem (RSS) revealed that while most have circular outer edges, almost all of the inner gap edges are measurably eccentric (Hedman et al. 2010; French et al. 2010). A detailed study of the edges of all eight gaps as well as the four dense ringlets within the Cassini Division by French et al. (2016a) utilized the full suite of stellar occultation data from VIMS and the Cassini Ultraviolet Imaging Spectrometer (UVIS), as well as an expanded set of RSS data. In addition to confirming the above results, this study revealed the existence of a large number of normal edge-modes, with $m$-values ranging from 0 to 13 and radial amplitudes between 0.20 and $7.6 \mathrm{~km}$. 


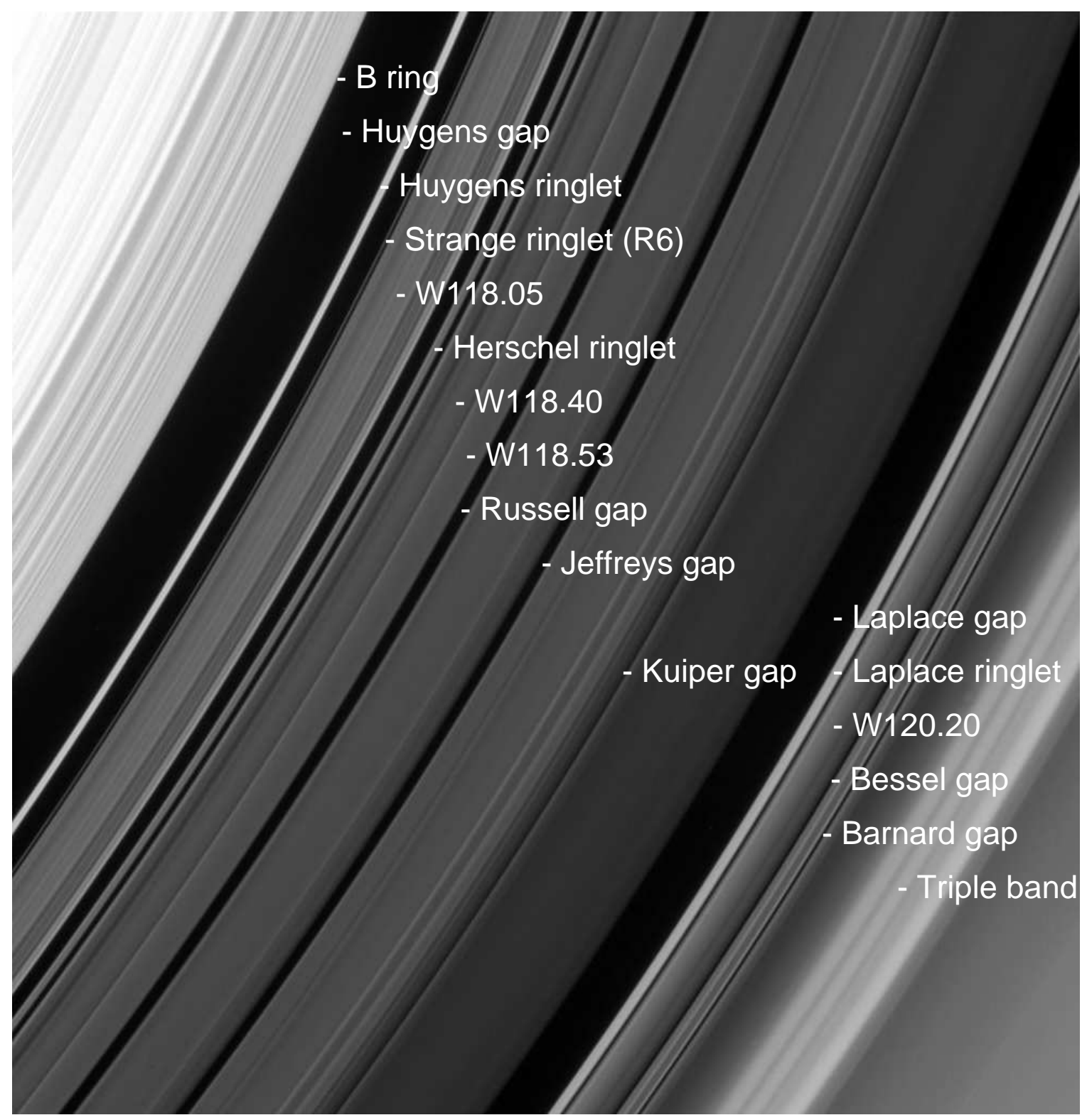

Figure 1. An overview of the Cassini Division with its named gaps and ringlets, from Cassini image N1495286873 (taken on 2005 May 20). The four waves that are the subject of this investigation are identified as W118.05, W118.40, W118.53 and W120.20. The projected ring plane resolution of the image is $4 \mathrm{~km} /$ pixel and the observer ring elevation is $-21.6^{\circ}$.

Besides the quasi-regular pattern of gaps with their inner/outer edge eccentricity patterns, the Cassini Division harbors at least seven narrow ringlets, four of which have optical depths equal to or exceeding that of the surrounding material. (The remaining three are faint, tenuous features generally detectable only in images (Porco et al. 2005; Colwell et al. 2009b); they do not appear in Fig. 2.) The narrow, opaque Huygens ringlet, located within the eponymous gap, was found by Turtle et al. (1991), Spitale et al. (2006) and Spitale and Hahn (2016) to exhibit both $m=1$ (i.e., elliptical) and $m=2$ perturbations, with the latter being attributed to the nearby 

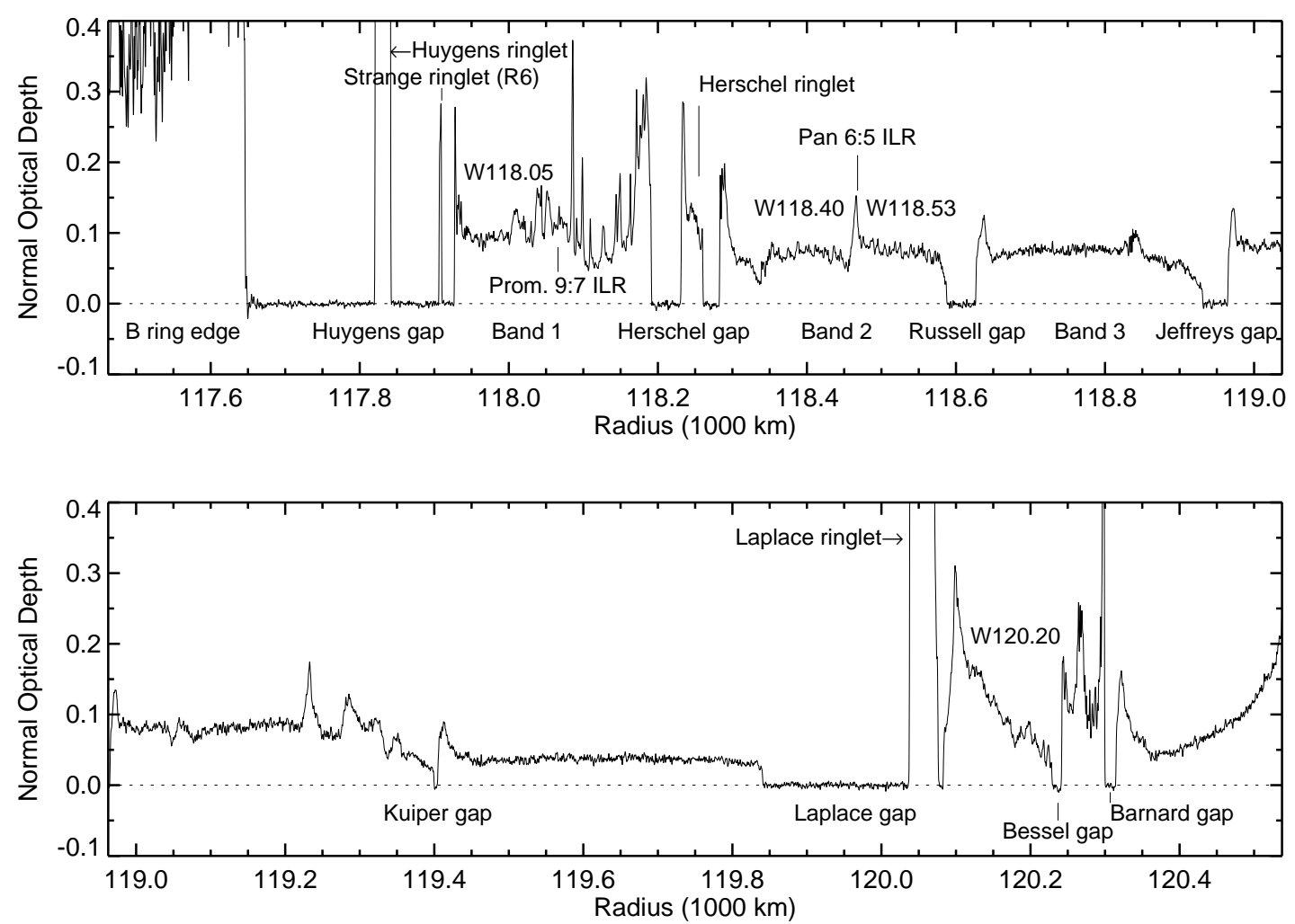

Figure 2. Prominent ringlets and gaps in the Cassini Division, as seen in an optical depth profile from the Cassini RSS egress occultation on rev 7. The wave features that are the focus of this work are illustrated: W118.05, W118.40, W118.53, and W120.20. The data are shown at a radial processing resolution of $1 \mathrm{~km}$.

Mimas 2:1 resonance. Also located within the Huygens gap is the very narrow R6 ringlet (sometimes referred to informally as the Strange ringlet, a moniker we adopt here as well). Although less than $2 \mathrm{~km}$ in width, this ringlet shows a wide variety of normal modes with $1 \leq m \leq 5$ as well as a significant inclination $(a \sin i=7.4 \mathrm{~km})$ with respect to the mean ring plane (French et al. 2016a).

Unlike the Huygens and R6 ringlets, the Herschel ringlet is comparable in optical depth to the surrounding material. In addition to showing several weak normal modes, its edges are both eccentric and inclined, although the pericenters of the inner and outer edges are not closely aligned (French et al. 2016a). Outermost of the narrow ringlets is the relatively opaque Laplace ringlet, which is eccentric but apparently not inclined.

In contrast to the gap and ringlet edges, there have been relatively few studies of the regions of the Cassini Division between the gaps, several of which exhibit significant internal structure. In this paper, we explore the properties of three regions of wavelike structure in the Cassini Division, as labelled in Fig. 2. First, we examine the structure in two broad bands in the inner Cassini Division, henceforth referred to as Bands 
1 and 2. This region was first investigated in detail by Marouf and Tyler (1986), who interpreted the observed fluctuations in optical depth seen in the single Voyager radio occultation profile as moonlet wakes, similar to those seen near the Encke gap (Showalter et al. 1986). They proposed that the wakes would be produced by a pair of unseen nearby satellites in the Herschel gap. In order to explain the relatively long wavelengths and large amplitudes of these structures, however, it was necessary to postulate that the satellites in question were located within $10-15^{\circ}$ of longitude of the occultation trace, and were of order $10 \mathrm{~km}$ in size.

In a subsequent analysis of Voyager imaging (ISS), radio occultation (RSS), and stellar occultation (PPS) data, Flynn and Cuzzi (1989) found instead that the wavelike structures in Bands 1 and 2, specifically those that we label below and in Fig. 2 as W118.05 and W118.40, were essentially axisymmetric, meaning that their radial wavelengths showed no significant variation with longitude, such as would be expected for moonlet wakes. Furthermore, they could find no evidence in the images for waves at the edges of the Herschel gap, such as those seen on the edges of the Encke and Keeler gaps in the A ring (Cuzzi and Scargle 1985), or for variations in radial wavelength across individual images. They therefore concluded that the moonlet wake model was very unlikely to be correct. Indeed, no such satellites were ever discovered in the Voyager images, nor have any been seen in the much more extensive Cassini imaging data set, despite several searches sensitive to km-size bodies (Nicholson et al. 2018).

Flynn and Cuzzi (1989) did, however, suggest an alternative interpretation of the structure in Band 1. Noting that both edges of this region showed evidence for radial perturbations with $m=1$ (i.e., they could be fit by freely precessing ellipses), and that the outward decrease in wavelength was consistent with that of a density wave with wavenumber $m=1$, they proposed that the wave in Band 1 might well be an outward-propagating density wave. Such a model implied a value for the local background surface mass density of $\sim 1 \mathrm{~g} \mathrm{~cm}^{-2}$, consistent with more recent determinations for the Cassini Division (Colwell et al. 2009a). Although they were unable to suggest a source for the wave, Flynn and Cuzzi (1989) also noted that the implied resonance radius was close to the apoapse of the eccentric Huygens ringlet. Using a much larger set of Cassini observations, we confirm that both Bands 1 and 2 are dominated by outward-propagating density waves with $m=1$. We further speculate that the origins of these waves are to be found in perturbations from the Huygens (or possibly Strange) and Herschel ringlets.

Second, we identify a somewhat less-prominent wavelike structure in the outer Cassini Division between the Laplace and Bessel gaps as a conspicuous example of a long-wavelength standing wave in a planetary ring system. It appears to be the result of the coaddition of an outward-propagating $m=1$ density wave, perhaps driven 
by the nearby Laplace ringlet, and its reflection at the inner edge of the Bessel gap. To our knowledge, this feature has not previously been discussed in print, although Borderies et al. (1985) predicted the presence of a standing wave inside the dense B ring, using a granular flow model and Spitale and Porco (2010) interpreted complex $m=1$ structure seen in Cassini images interior to the outer B ring edge as evidence of a standing wave.

We organize our results as follows. In Section 2, we present a brief overview of the observations used in our investigation and describe the appearance and regional context of the waves that are the focus of our study. We review our techniques for identifying wave properties in Section 3, and Section 4 contains our investigation of the wave structure in the inner Cassini Division, in what we refer to above as Bands 1 and 2. From measurements of individual wave crests and from wavelet analysis of the overall wave structure, we identify two prominent $m=1$ ILR-type waves, and explore possible wave generation mechanisms. In Section 5 we investigate, but ultimately reject, the possibility that these waves are bending rather than density waves. In Section 6, we characterize the standing wave in the outer Cassini Division, and describe the circumstances required for standing waves to exist in the rings. Finally, in Section 7, we summarize our results and pose a series of unanswered questions to be addressed in the future.

\section{OVERVIEW OF THE REGIONS STUDIED}

Figure 2 shows an occultation profile of the Cassini Division, with the individual gaps and ringlets labeled. ${ }^{1}$ The regions studied in the present paper are those between the Huygens and Herschel gaps, referred to as Band 1 by Flynn and Cuzzi (1989); that between the Herschel and Russell gaps, referred to by them as Band 2; and the unnamed ramp-shaped region between the Laplace and Bessel gaps. For the sake of brevity, and to be consistent with the notation applied to waves in the $\mathrm{C}$ ring by Colwell et al. (2009b), Hedman and Nicholson (2013) and later authors, we will refer to the waves in these three regions by their mean radii in thousands of $\mathrm{km}$, as W118.05, W118.40/W118.53 and W120.20, respectively. (The waves in the inner and outer parts of Band 2 have somewhat different characteristics, so we assign them individual names.)

Of the three regions studied here, the structure in Band 1, bounded by the Huygens and Herschel gaps and located between radii of 117,930 and $118,190 \mathrm{~km}$, is the most complex. Figure 3 shows a set of RSS occultation profiles of this region, offset

\footnotetext{
${ }^{1}$ The eight gaps have been officially named by the IAU Committee on Solar System Nomenclature; we
} adopt the same names for their embedded ringlets, following the practice of French et al. (2016a). 
vertically for clarity and sorted and labeled by true anomaly ${ }^{2}$, where we have assumed a local mean apsidal precession rate of $\dot{\varpi}=5.000^{\circ} \mathrm{d}^{-1}$, with a corresponding orbital radius of $\sim 117,957 \mathrm{~km}$. Note that this rate also does a reasonable job of displaying the eccentric inner edge of the Herschel gap, whose measured precession rate is $\dot{\varpi}=$ $4.9736^{\circ} \mathrm{d}^{-1}$ (French et al. 2016a). Starting at the inner edge of Band 1, and extending out to $\sim 118,080 \mathrm{~km}$, we see a series of six or seven smooth, low-amplitude oscillations with an average wavelength of $\sim 15 \mathrm{~km}$. The systematic progression of peak locations to smaller radii with increasing true anomaly (cf. Fig. 8) is indicative of a trailing spiral pattern. This is the first region studied by Marouf and Tyler (1986) and by Flynn and Cuzzi (1989).

The outer third of Band 1, however, exhibits a more irregular profile with a series of sharp peaks whose structure varies systematically as a function of the local true anomaly, but whose geometry is neither clearly trailing nor leading. Some isolated spikes in optical depth, such as those at $\sim 118,090 \mathrm{~km}$ and $\sim 118,150 \mathrm{~km}$, are prominent only over a limited range in true anomaly. Separating these two portions of Band 1 is the relatively weak density wave driven by the Prometheus 9:7 ILR at $\sim 118,066 \mathrm{~km}$ (Colwell et al. 2009b) (labeled in Fig. 2), though this wave is not well-resolved at the $1 \mathrm{~km}$ resolution of the profiles in Fig. 3. In addition to the 10$20-\mathrm{km}$ scale wavelike structure seen over most of Band 1, which is the subject of our study, there are zones of much finer-scale structure near both inner and outer edges, with wavelengths as short as 1-2 km. The oscillations near the outer edge, between 118,170 and 118,190 km, were interpreted by Flynn and Cuzzi (1989) as a possible wake driven by a nearby km-size moonlet in the Herschel gap.

Figure 4 shows the same set of RSS occultation profiles for Band 2, again sorted by true anomaly, but here with $\dot{\varpi}=4.950^{\circ} \mathrm{d}^{-1}$, with a corresponding orbital radius of $\sim 118,289 \mathrm{~km}$. Band 2 is bounded by the Herschel and Russell gaps and located between radii of 118,283 and $118,590 \mathrm{~km}$. The eccentric inner edge of the Russell gap has a measured precession rate of $\dot{\varpi}=4.9092^{\circ} \mathrm{d}^{-1}$ (French et al. 2016a) and is readily seen at the right edge of the figure. The structure in Band 2 is somewhat simpler than that in Band 1. The inner half of the region - which we identify here as wave W118.40 - shows a series of seven or eight regular undulations with an average wavelength of $\sim 10 \mathrm{~km}$, while the outer half - here designated as W118.53 - shows a set of $\sim 15$ higher-frequency oscillations. In the inner zone the pattern of peaks in optical depth clearly form a trailing spiral pattern, similar to that in Band 1 - slanting dotted vertical lines to guide the eye approximately follow the decreasing orbital radius with increasing true anomaly, for several adjacent wavecrests. Also as in Band 1, these two sub-regions are separated by a weak density wave; in this case

${ }^{2}$ The true anomaly $f$ is the angular distance of the occultation ring intercept point from the nominal pericenter of a precessing, eccentric orbit at the local distance from Saturn. In terms of the inertial longitude $\lambda$ and observation time $t$, it is given by $f=\lambda-\lambda_{0}-\dot{\varpi}\left(t-t_{0}\right)$, where $\dot{\varpi}$ is the local apsidal precession rate, $t_{0}$ is the reference epoch of 2008 Jan 1.5 UTC., and $\lambda_{0}$ is the inertial longitude of periapse at the epoch. 
FRENCH ET AL.

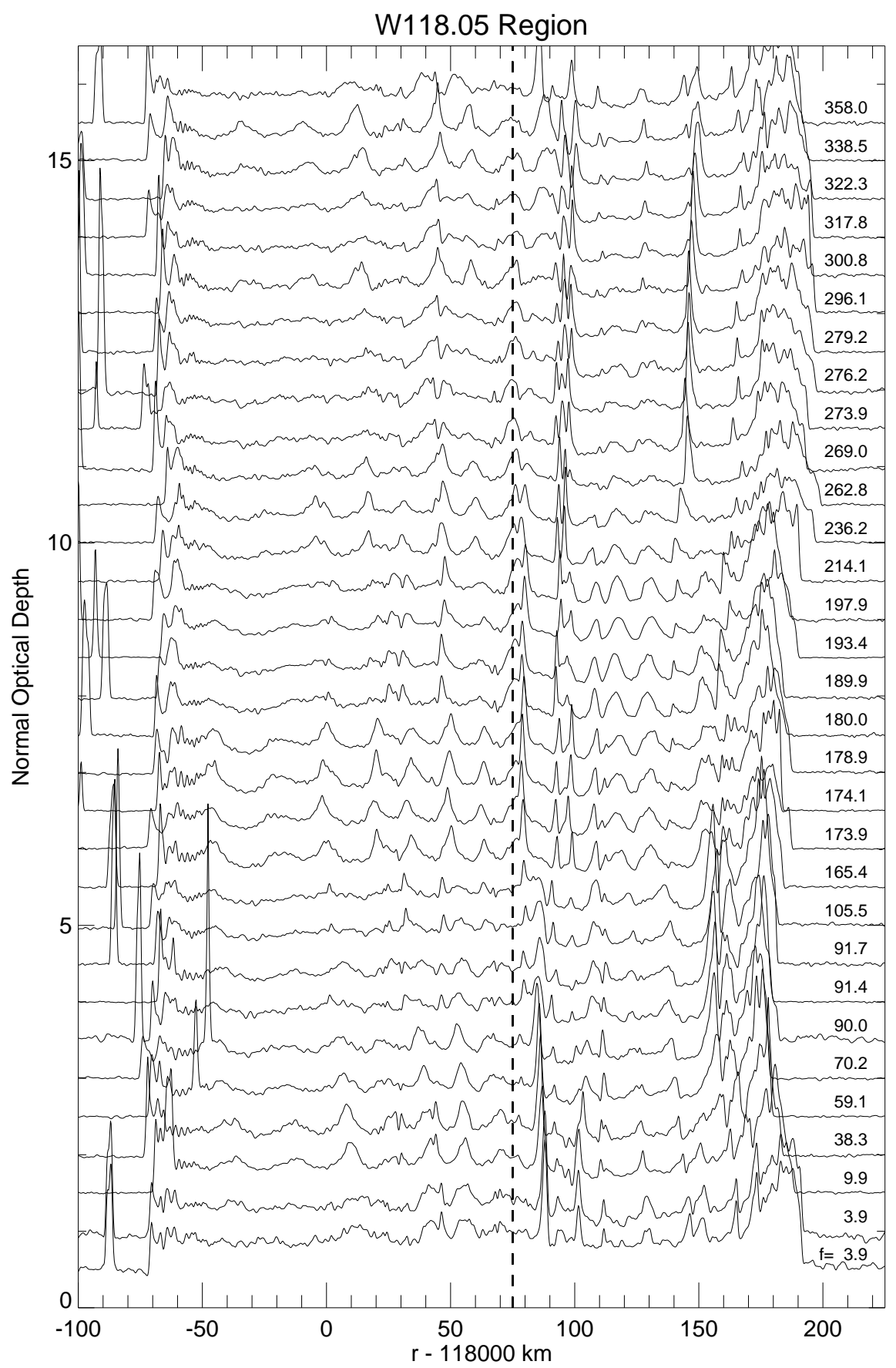

Figure 3. Optical depth profiles of wave W118.05 in Band 1, offset vertically for clarity and sorted by true anomaly $f$ (labeled at right) for an assumed pattern speed of $5.000^{\circ} \mathrm{d}^{-1}$. The data are from X-band RSS occultations on revs $7-67$, processed at a radial resolution of $1 \mathrm{~km}$. The narrow R6 (or Strange) ringlet and the outer edge of the Huygens gap are seen at the left edge of the plot, while the eccentric inner edge of the Herschel gap is seen at the right edge. The latter has a substantial radial amplitude of $a e=8.3 \mathrm{~km}$. Only barely visible at this scale and resolution is the weak Prometheus 9:7 ILR density wave outward of its resonance radius of $\sim 118,075 \mathrm{~km}$, marked by a vertical dashed line. 
due to the Pan 6:5 ILR at $\sim 118,453 \mathrm{~km}$ (Colwell et al. 2009b). (The wave itself is unresolved at the resolution of Fig. 4, but appears as a prominent peak in optical depth at $\sim 118,465 \mathrm{~km}$, as labeled in Fig. 2.) Across Band 2, the average radial wavelength decreases smoothly from $\sim 12 \mathrm{~km}$ at the inner edge to $\sim 5 \mathrm{~km}$ at the outer edge. The studies by Marouf and Tyler (1986) and Flynn and Cuzzi (1989) both focussed on the structure in the inner half of Band 2, between radii of 118,350 and $118,450 \mathrm{~km}$.

The third of our wave features, W120.20, is located in the band between the Laplace and Bessel gaps, between radii of 120,086 and $120,231 \mathrm{~km}$, in a region where the background optical depth is strongly decreasing. Figure 5 shows a sequence of RSS occultation profiles of this region, again sorted by true anomaly, this time with $\dot{\varpi}=4.720^{\circ} \mathrm{d}^{-1}$, with a corresponding orbital radius of $\sim 119,872 \mathrm{~km}$. Visible at the right edge of the figure is the eccentric inner edge of the Bessel gap, which has a measured precession rate of $\dot{\varpi}=4.6845^{\circ} \mathrm{d}^{-1}$, as determined from fits to multiple occultation measurements (French et al. 2016a). The eccentricity is difficult to see at the scale of this figure. Wavelike structure is only apparent to the eye in the outer half of the band, outside $\sim 120,180 \mathrm{~km}$, but wavelet analysis (see Section 6) clearly shows that it extends inward at least to $120,140 \mathrm{~km}$. Just as for the inner region of the W118.05 wave $(117,970-118,060 \mathrm{~km})$ and the W118.40/W118.53 complex, the radial wavelength of the W120.20 wave clearly decreases outwards, in this case from perhaps $25 \mathrm{~km}$ at the inner edge of the band to $\sim 5 \mathrm{~km}$ at the outer edge. But unlike the waves in Bands 1 and 2, the W120.20 wave is neither clearly trailing nor leading, a curious fact to which we will return in Section 6.1. This wave does not seem to have been the subject of any previous published studies.

\section{IDENTIFYING WAVELIKE STRUCTURE}

We begin by assuming that each wave is a spiral density wave with an azimuthal wavenumber $m$, rotating relative to inertial space at an angular velocity or pattern speed $\Omega_{p}$. As long as such a wave is weak enough to remain in the linear regime, the perturbed surface density can be written as the real part of

$$
\sigma(r, \lambda, t)=\sigma_{0}\left[1+A(r) e^{i\left(\phi_{r}(r)+\phi_{\lambda, t}\right)}\right]
$$

where $\sigma_{0}$ is the background surface mass density and $A(r)$ is a slowly-varying amplitude factor. The phase of the wave is composed of both a rapidly-varying radial function $\phi_{r}(r)$ that describes the oscillatory part of the wave, plus a more slowlyvarying azimuthal phase factor given by (Shu 1984)

$$
\phi_{\lambda, t}=|m|\left[\lambda-\lambda_{0}-\Omega_{p}\left(t-t_{0}\right)\right]
$$

Here, $\lambda$ and $t$ are the longitude and time at which the occultation track crossed the wave, $t_{0}$ is a reference epoch $(=2008$ Jan $1.5 \mathrm{UTC})$, and $\lambda_{0}$ is a reference longitude. 


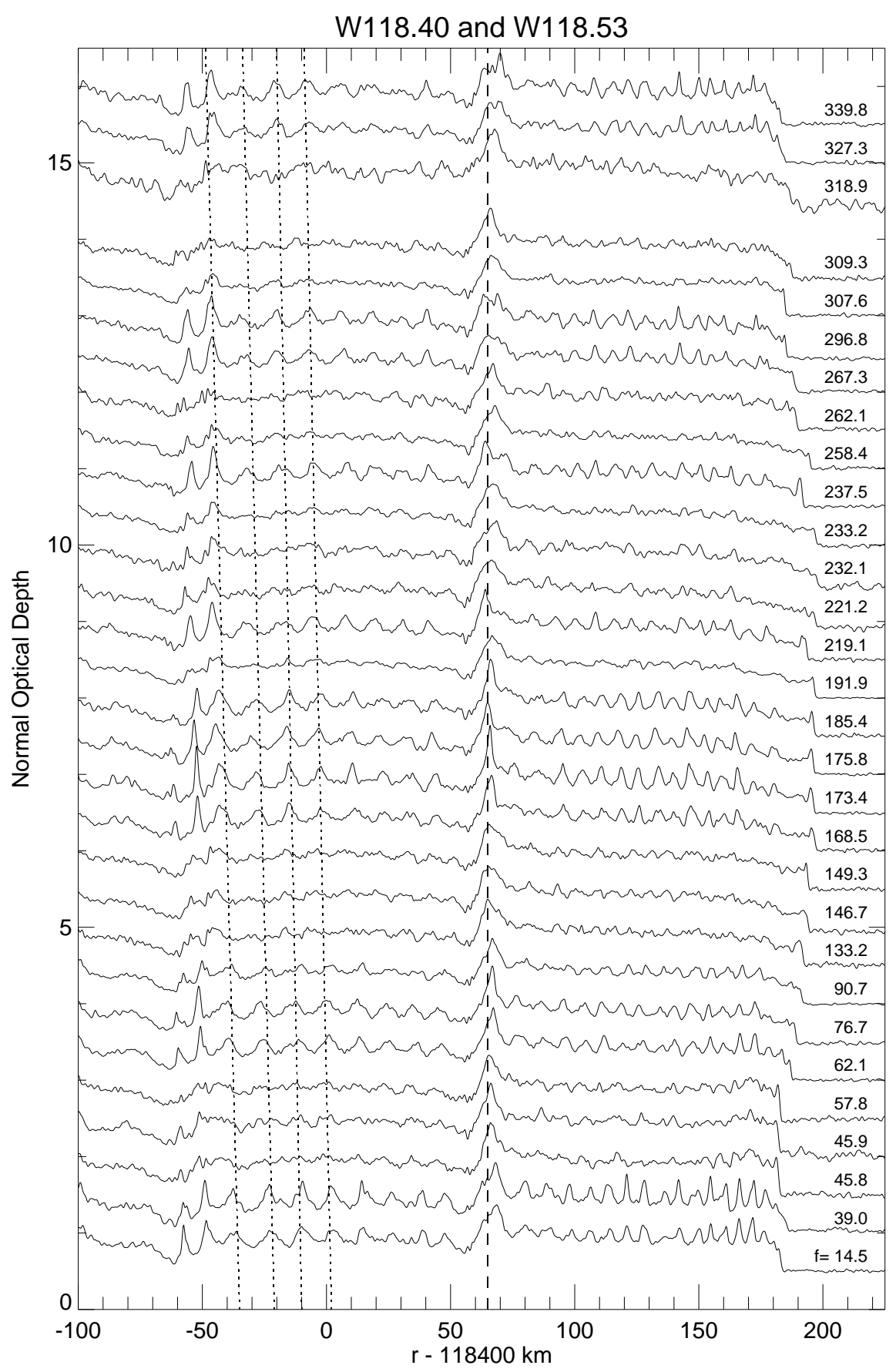

Figure 4. Optical depth profiles of waves W118.40 and W118.53 in Band 2, offset vertically for clarity and sorted by true anomaly $f$ (labeled at right) for an assumed pattern speed of $4.950^{\circ} \mathrm{d}^{-1}$. The data are from X-band RSS occultations on revs $7-67$, processed at a radial resolution of $1 \mathrm{~km}$. The eccentric inner edge of the Russell gap, with a radial amplitude $a e=7.6 \mathrm{~km}$, is seen at the extreme right edge of the plot. The slanted dotted lines approximately trace the trend of decreasing radius with increasing true anomaly of several wavecrests in the inner region of W118.40. Visible as a narrow peak in optical depth separating the two wave regions is the Pan 6:5 ILR density wave at $\sim 118,465 \mathrm{~km}$, marked by a vertical dashed line. 


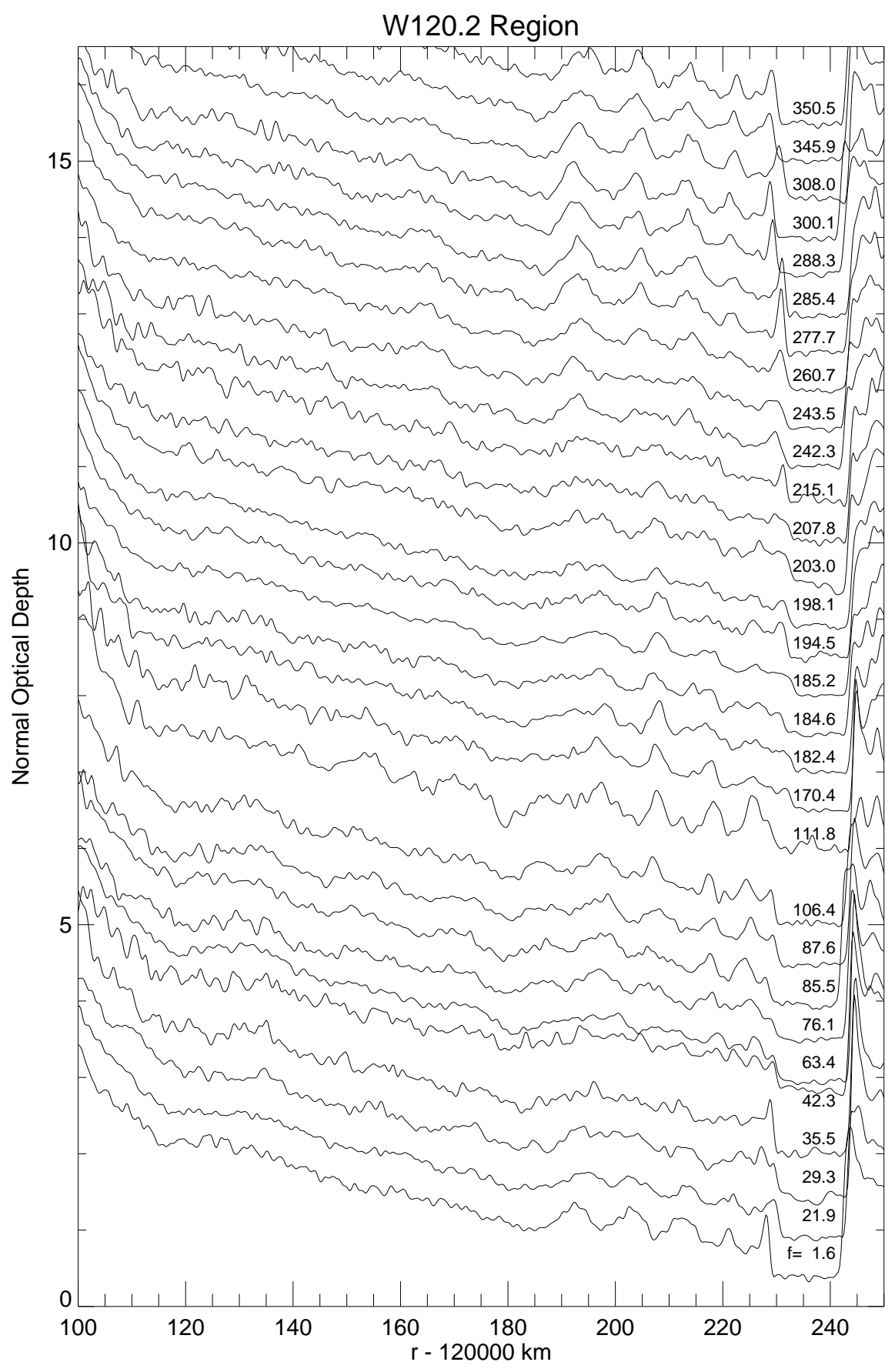

Figure 5. Optical depth profiles of wave W120.20 in the outer Cassini Division, offset vertically for clarity and sorted by true anomaly $f$ (labeled at right) for an assumed pattern speed of $4.720^{\circ} \mathrm{d}^{-1}$. The data are from X-band RSS occultations on revs $7-67$, again processed at a radial resolution of $1 \mathrm{~km}$. The Bessel gap and its eccentric inner edge, with a radial amplitude of $a e=1.8 \mathrm{~km}$, is seen at the right edge of the plot.

With this formulation the radial phase $\phi_{r}$ of such a wave is assumed to be the same at all longitudes and times. As in our previous work (Hedman and Nicholson 2013, 2014; French et al. 2016a), we denote ILR-type density waves by positive values of $m$ and Outer Lindblad Resonance (OLR-type) waves by negative values of $m$. With 
this convention, the pattern speeds of both types of wave are given by the common expression

$$
m \Omega_{p}=(m-1) n+\dot{\varpi}_{\mathrm{sec}},
$$

where $n$ is the local Keplerian mean motion and $\dot{\varpi}_{\text {sec }}$ is the apsidal precession rate due to secular perturbations (primarily Saturn's $J_{2}$ and $J_{4}$ ). For waves with $m=1$, such as those encountered here, we have $\Omega_{p}=\dot{\varpi}_{\text {sec }}$. For a wave with a specified pattern speed and $m$-value, the resonant radius is the location where Eq. (3) is satisfied exactly.

At some distance from the resonance, the radial wavenumber $k(r)=d \phi_{r} / d r$ for freely-propagating density waves is given by the asymptotic expression (Shu 1984; Hedman and Nicholson 2013):

$$
|k(r)| \simeq \frac{\left[3(m-1)+\frac{21}{2} J_{2}\left(R_{p} / r_{L}\right)^{2}\right] M_{p}}{2 \pi \sigma_{0} r_{L}^{4}}\left(r-r_{L}\right),
$$

where $r_{L}$ is the resonant radius, $M_{p}$ is the mass of the central planet, $J_{2}$ is the zonal gravity harmonic, and $R_{p}$ is the reference radius for $J_{2}$. ILR-type density waves $(m>0)$ propagate outwards from an ILR, while OLR-type density waves $(m \leq 0)$ propagate inwards from an OLR. In both cases, $|k|$ increases linearly with distance from $r_{L}$. Referring back to Eqns. (1) and (2), we see that $k(r)>0$ corresponds to a trailing spiral wave (i.e., the radius of a wave crest decreases with increasing longitude) while $k(r)<0$ for a leading spiral, a fact we will make use of in Section 6.1 below. The corresponding radial phase is given by

$$
\phi_{r}(r)-\phi_{0}=\int_{r_{L}}^{r} k\left(r^{\prime}\right) d r^{\prime} \simeq \pm \frac{1}{2} K\left(r-r_{L}\right)^{2},
$$

where $\phi_{0}$ is a constant, $K$ refers to the constant factor in Eq. (4), and the \pm sign describes trailing and leading waves, respectively. For $m=1$, the factor of $3(m-1)$ in the expression for $k(r)$ is zero and in this case the radial wavelength is significantly longer than for other values of $m .^{3}$

In general, our goal is to combine data from multiple occultations to determine both the azimuthal wavenumber $m$ and the pattern speed $\Omega_{p}$ for each wave, and then to compare these with the above theoretical expressions. In order to identify the nature of the wavelike structure such as that in Bands 1 and 2, we first convert each occultation-derived optical depth profile into a wavelet profile, using the same procedure described by Hedman and Nicholson $(2013,2014)$ to analyze unknown waves in the $\mathrm{C}$ ring. We denote the wavelet transform of profile $i$ by $\mathcal{W}_{i}(r, k)$, where $r$ is radius and $k$ is the radial wavenumber. $\mathcal{W}_{i}$ is a complex quantity, with both an amplitude

\footnotetext{
3 This is because, as noted above, the pattern speed is much lower for waves with $m=1$ and the ring's self-gravity is only required to compensate for the radial gradient in the apsidal precession rate $\dot{\varpi}$ rather than for that in the mean motion $n$.
} 
$\mathcal{A}_{i}(r, k)$ and a phase $\Phi_{i}(r, k)$. We use two methods in the current work. In the first, which follows that employed by Hedman and Nicholson (2013, 2014), French et al. (2016b) and French et al. (2019), we compare the difference in the measured phase for pairs of observations $\delta \Phi_{i, j}=\Phi_{i}-\Phi_{j}$ with the difference predicted by Eq. (2), which we denote as $\delta \phi_{i, j}$

$$
\delta \phi_{i, j}=|m|\left[\delta \lambda-\Omega_{p} \delta t\right]
$$

where $\delta \lambda$ and $\delta t$ are the differences in longitude and time between the two occultation profiles at the radius of interest. This procedure involves subtracting the phases of the two wavelet transforms, $\mathcal{W}_{i}$ and $\mathcal{W}_{j}$, so that their implicit common radial dependence described by $\phi_{r}$ cancels out, and then averaging over specified ranges of $r$ and $k$. For further details the reader is referred to the above papers. We then sum the squares of $\delta \Phi_{i, j}-\delta \phi_{i, j}$ over all distinct pairs of occultations and scan over a range of values of $\Omega_{p}$ to find the pattern speed corresponding to the minimum RMS phase difference for a specified value of $m$. Plotting the RMS value of $\delta \Phi_{i, j}-\delta \phi_{i, j}$ vs $\Omega_{p}$ provides a useful visual way to judge the significance of the best-fitting model. In general, only a single value of $m$ will yield an acceptably small value of the RMS phase difference.

In the second method, as developed by Hedman and Nicholson (2016) and also employed by Hedman et al. (2019) and Hedman and Nicholson (2019), the wavelet transform for each occultation is first corrected to zero longitude and to the adopted epoch using trial values of $m$ and $\Omega_{p}$ via the expression

$$
\mathcal{W}_{\phi, j}(r, k)=\mathcal{W}_{j} e^{-i \phi_{j}}=\mathcal{A}_{j}(r, k) e^{i\left(\Phi_{j}-\phi_{j}\right)},
$$

where we denote the predicted wave phase $\phi_{\lambda, t}$ by $\phi_{j}$. These phase-corrected wavelets are then summed over all occultations to obtain an average wavelet, as if all observations had been made at zero longitude and at time $t_{0}$

$$
\left\langle\mathcal{W}_{\phi}(r, k)\right\rangle=\frac{1}{N} \sum_{j} \mathcal{W}_{\phi, j}(r, k)
$$

where $N$ is the number of occultations combined. In this case, the assumed pattern speed is varied until a maximum in the power of the average phase-corrected wavelet is obtained for the specified value of $m$

$$
\mathcal{P}_{\phi}(r, k)=\left|\left\langle\mathcal{W}_{\phi}\right\rangle\right|^{2}=\left|\frac{1}{N} \sum_{j} \mathcal{W}_{\phi, j}(r, k)\right|^{2} .
$$

Finally, this phase-corrected power is divided by the average uncorrected wavelet power

$$
\overline{\mathcal{P}}(r, k)=\left\langle\left|\mathcal{W}_{j}\right|^{2}\right\rangle=\frac{1}{N} \sum_{j}\left|\mathcal{W}_{j}(r, k)\right|^{2} .
$$

It can be shown (Hedman and Nicholson 2016) that this normalized power, $\mathcal{R}(r, k)=$ $\mathcal{P}_{\phi}(r, k) / \overline{\mathcal{P}}(r, k)$ lies between 0 and 1 , depending on the accuracy of $\Omega_{p}$ and whether or 
not multiple waves are present. Plotting the normalized power vs both $r$ and $k$ shows both the radial extent and the varying radial wavenumber of the wave, while plotting the power $\mathcal{P}_{\phi}(r, k)$ averaged over $k$ vs $r$ and $\Omega_{p}$ provides an idea of the uncertainty in pattern speed and the probable location of the Lindblad resonance driving the wave, i.e., the location at which Eq. (3) is satisfied.

One advantage of this procedure is that, for the wrong value of $\Omega_{p}$ and given many occultations, the average wavelet and its power are both close to zero. A second advantage is that, unlike the first procedure, it can handle situations where more than one wave is present at the same radius. A final advantage is that the combined phase-corrected wavelet may be inverted to obtain an average profile of the wave, with the phase differences between individual occultation profiles removed.

In the following sections, we will employ both of these procedures in order to determine the best-fitting values of $m$ and $\Omega_{p}$ for each wave, using different subsets of occultation data.

\section{WAVE STRUCTURE IN THE INNER CASSINI DIVISION}

Our first step in unravelling the wavelike structure in Bands 1 and 2 was to apply the phase-corrected wavelet procedure, using a large set of VIMS stellar occultation profiles spanning the range from 2006 (R Hya, rev 36) through early 2017 ( $\alpha$ Ori, rev 269). This is the same set of 56 occultations listed in Table 1 of French et al. (2019). Experiments in which the azimuthal wavenumber $m$ was varied from -10 to 10 showed significant power in the phase-corrected wavelet only for $m=1$, in both regions. The results for $m=1$ are shown in Fig. 6 .

The successive panels in this rather complicated figure are as follows. From top to bottom, panel (i) shows a representative optical depth profile of the region, for context; here, it is the RSS egress occultation on rev 7. Panel (ii) shows the average uncorrected wavelet power $\overline{\mathcal{P}}$ as a greyscale function of radius and radial wavenumber. ${ }^{4}$ The most prominent features here are associated with the sharp edges of gaps and ringlets that generate significant high spatial frequency power. Panel (iii) shows the average phase-corrected wavelet power $\mathcal{P}_{\phi}$, again as a greyscale function of radius and radial wavenumber. At each radius, the assumed pattern speed is that given by Eq. (3), for the specified value of $m$. The sharp edges are now somewhat suppressed, leaving the wave structure more obvious. Both panels are displayed on a common logarithmic scale, because of their large dynamic range. Panel (iv) shows the ratio $\mathcal{R}=\mathcal{P}_{\phi} / \overline{\mathcal{P}}$ on a linear scale from 0 to 1 , which highlights the fraction of the average

\footnotetext{
4 This panel is similar in format to the regional wavelet power spectra computed by Tiscareno and Harris (2018) from high-resolution Cassini images across the entire ring system (see their online Supplementary Material). However, the focus of their work was to identify waves associated with known satellite resonances, and the waves we identify here are inconspicuous and not identified in their derived radial $I / F$ scans or wavelet spectra.
} 
power associated with the $m=1$ perturbation. Here we see significant power only in Bands 1 and 2, with wavenumbers in the range $0.2 \leq k \leq 0.8 \mathrm{~km}^{-1}$, or radial wavelengths of $7.5-30 \mathrm{~km}$. In panel (v), we vary the assumed pattern speed (expressed here as the corresponding shift in the resonant radius, $r_{L}$ ) for each radius and then plot the peak value of the power ratio $\mathcal{R}$ within a specified range of $k$. For example, a maximum in the peak power at $\delta r=-200 \mathrm{~km}$ at a particular radius means that the best-fitting pattern speed at that radius corresponds to a resonant radius that is $200 \mathrm{~km}$ less than the local value. Panel (vi) summarizes the best-fitting pattern speed and resonant radius at each location in panel $(\mathrm{v})$ where the power ratio is appreciable. These were obtained by fitting a 1D gaussian model to $\mathcal{R}$ as a function of $\delta r$, separately for each radius, and then converting the central value of $r_{L}$ for each wave back to a pattern speed. Dotted lines show the average values of $r_{L}$ for each region.

Focussing first on the third and fourth panels in Fig. 6, we see strong evidence for $m=1$ ILR-type waves in Band 1, at radii corresponding to wave W118.05, and also in Band 2 spanning waves W118.40 and W118.53. In the case of W118.05, there is a weak indication that $k$ does increase outwards, as expected from Eq. (4). For waves W118.40 and W118.53, on the other hand, there is a clear positive gradient in $k$, at least between radii of 118,350 and $118,550 \mathrm{~km}$. Turning now to the fifth panel, we see that the best-fitting pattern speeds are significantly faster than the local values for both regions. For W118.05, the peak power occurs for $\delta r \simeq-150 \mathrm{~km}$, or $r_{L} \simeq 117,900 \mathrm{~km}$, with an average pattern speed of $5.010 \pm 0.008^{\circ} \mathrm{d}^{-1}$. For W118.40 and W118.53, the peak power occurs for $\delta r \simeq-250 \mathrm{~km}$, or $r_{L} \simeq 118,200 \mathrm{~km}$, with an average pattern speed of $4.963 \pm 0.008^{\circ} \mathrm{d}^{-1}$. The estimated uncertainties in pattern speed translate into an uncertainty in the resonant radii for these waves of $\frac{2}{7}\left(\delta \Omega_{p} / \Omega_{p}\right) r_{L} \simeq 50 \mathrm{~km}$, since $\Omega_{p}=\dot{\varpi}_{\mathrm{sec}} \propto r^{-7 / 2}$ for $m=1$.

Although the phase-corrected wavelet analysis is the most efficient way to search a large region for significant wave signals, it is less precise than the pairwise comparison method in specifying the best-fitting pattern speed. Our next step was therefore to apply the pairwise phase comparison method separately to each of the three waves in the inner Cassini Division, under the assumption that the wave in question has $m=1$. Our results are shown in Fig. 7, with a separate panel for each wave.

The W118.05 wave gives a deep minimum in the RMS phase residuals of $38.9^{\circ}$ at $\Omega_{p}=5.005^{\circ} \mathrm{d}^{-1}$. The corresponding resonant radius is $\sim 117,925 \mathrm{~km}$, about $37 \mathrm{~km}$ larger than but generally consistent with the peak-power result in Fig. 6. The radial range fitted was restricted to $117,990-118,090 \mathrm{~km}$, where the wave signature is clearest in Fig. 3, and the range of wavelengths averaged over was set at $5-25 \mathrm{~km}$.

Wave W118.40 yields a minimum RMS phase residual of $51.1^{\circ}$ at $\Omega_{p}=4.958^{\circ} \mathrm{d}^{-1}$. The corresponding resonant radius is $118,232.9 \mathrm{~km}$, about $30 \mathrm{~km}$ larger than but generally consistent with the peak-power result in Fig. 6. The radial range fitted was 


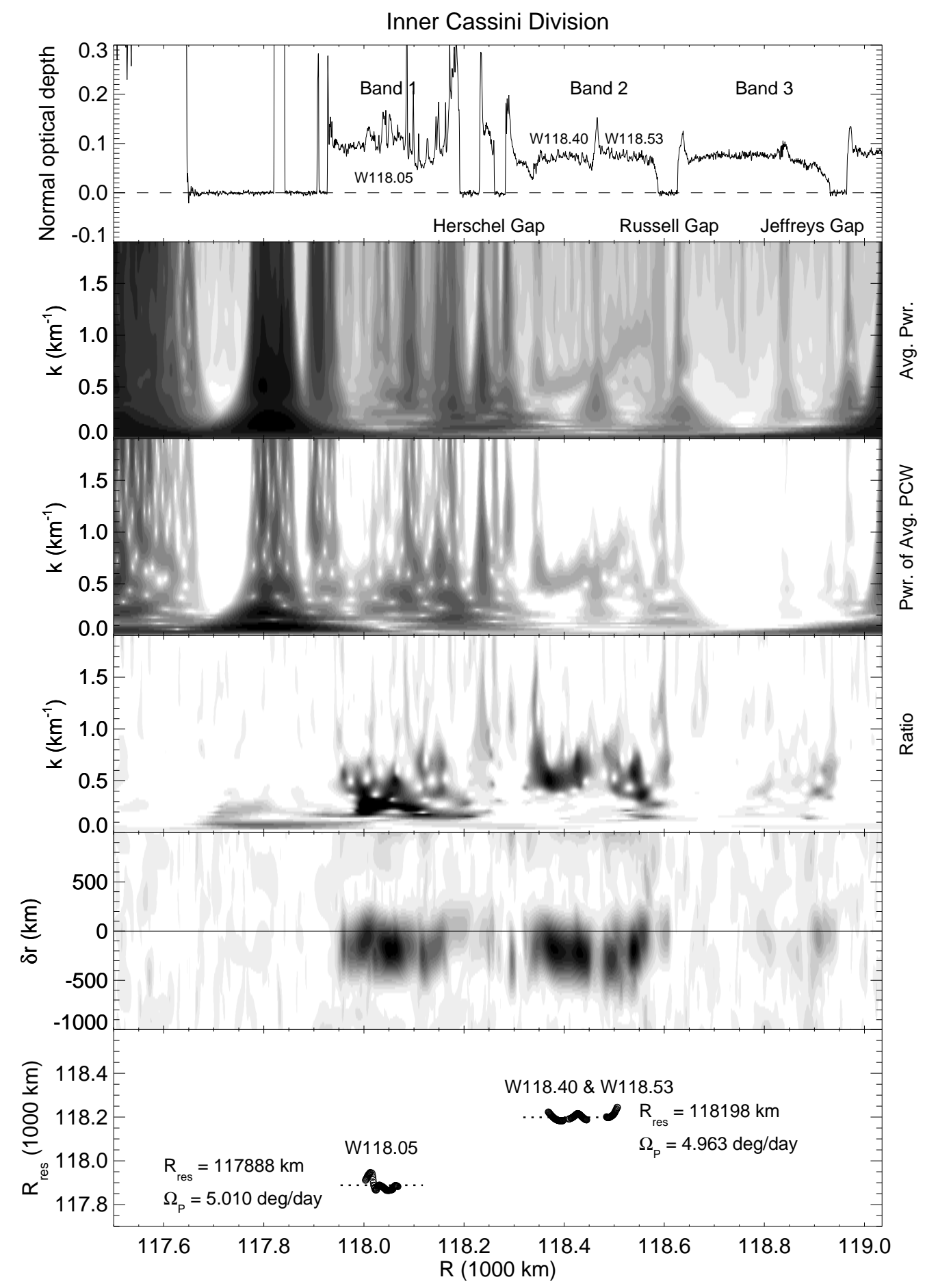

Figure 6. Phase-corrected wavelet analysis for Bands 1,2 and 3, assuming that the wave structure is due to density waves with $m=1$. See text for a detailed description of each panel. Note that significant power with $m=1$ is seen only in Bands 1 and 2 , with the best-fitting pattern speeds and resonant radii shown in the bottom panel. 
restricted to $118,360-118,440 \mathrm{~km}$ and the range of wavelengths averaged over was set at $5-20 \mathrm{~km}$.

The fit for wave W118.53 yields a weaker detection, with a minimum RMS phase residual of $83.8^{\circ}$ at $\Omega_{p}=4.964^{\circ} \mathrm{d}^{-1}$. The corresponding resonant radius is $118,195.2 \mathrm{~km}$, very close to the peak-power result of $118,198 \mathrm{~km}$ in Fig. 6. The radial range fitted was $118,490-118,570 \mathrm{~km}$ and the range of wavelengths averaged over was restricted to $3-10 \mathrm{~km}$, in an attempt to improve the fit.

For each of waves W118.05 and W118.40/118.53 the best-fitting pattern speeds from the pairwise phase comparison of $\delta \Phi_{i, j}-\delta \phi_{i, j}$ agree with those derived from the phase-corrected wavelets to within $0.005^{\circ} \mathrm{d}^{-1}$, less than the estimated uncertainty in the latter values. Recalling that $\Omega_{p}=\dot{\varpi}_{\text {sec }} \propto r^{-7 / 2}$ for $m=1$, we estimate that the differences in resonant radii are $\pm 35 \mathrm{~km}$. At this level of accuracy, the pattern speeds and resonant radii for W118.40 and W118.53 may be considered to be the same, suggesting that they are parts of a single wavetrain. We now look at the possible origins for each of these waves.

\subsection{W118.05 wave}

Based on the results in Figs. 6 and 7 above, this wave is provisionally identified as an ILR-type density wave with $m=1, \Omega_{p}=5.006 \pm 0.005^{\circ} \mathrm{d}^{-1}$, and a resonant radius of $117,920 \pm 35 \mathrm{~km}$. This places the likely source of the wave close to the outer edge of the Huygens gap, which has a mean radius of $117,931 \mathrm{~km}$, and to the Strange ringlet at $117,908 \mathrm{~km}$ (French et al. 2016a). However, neither the series of occultation profiles in Fig. 3 nor the wavelet spectra in Fig. 6 show a very clear trend of radial wavelength decreasing with increasing radius over the full radial extent of the region, as expected for an ILR-type density wave. In an attempt to verify the nature of this wave, we also measured the locations of individual wave crests in the same sequence of RSS occultation profiles shown in Fig. 3. Although a small fraction of our complete data set, these profiles all have a uniform and high signal-to-noise ratio conducive to such measurements. The results are shown in Fig. 8, with each set of measurements shown twice to facilitate linking successive crests in longitude. From this plot we see that (i) the great majority of measured crests in the range 117,950 - 118,080 km do indeed fit a model of a trailing spiral pattern (i.e., the radius of each crest decreases monotonically with increasing true anomaly $f=\lambda-\varpi$ ), (ii) the spiral is an $m=1$, as may be seen by observing that each 'arm' links to the next one after $360^{\circ}$ of longitude, (iii) the radial wavelength does seem to decrease outwards, as expected, and (iv) the scatter in the measured crest locations with respect to a simple linear spiral is only a few $\mathrm{km}$, in most cases. 

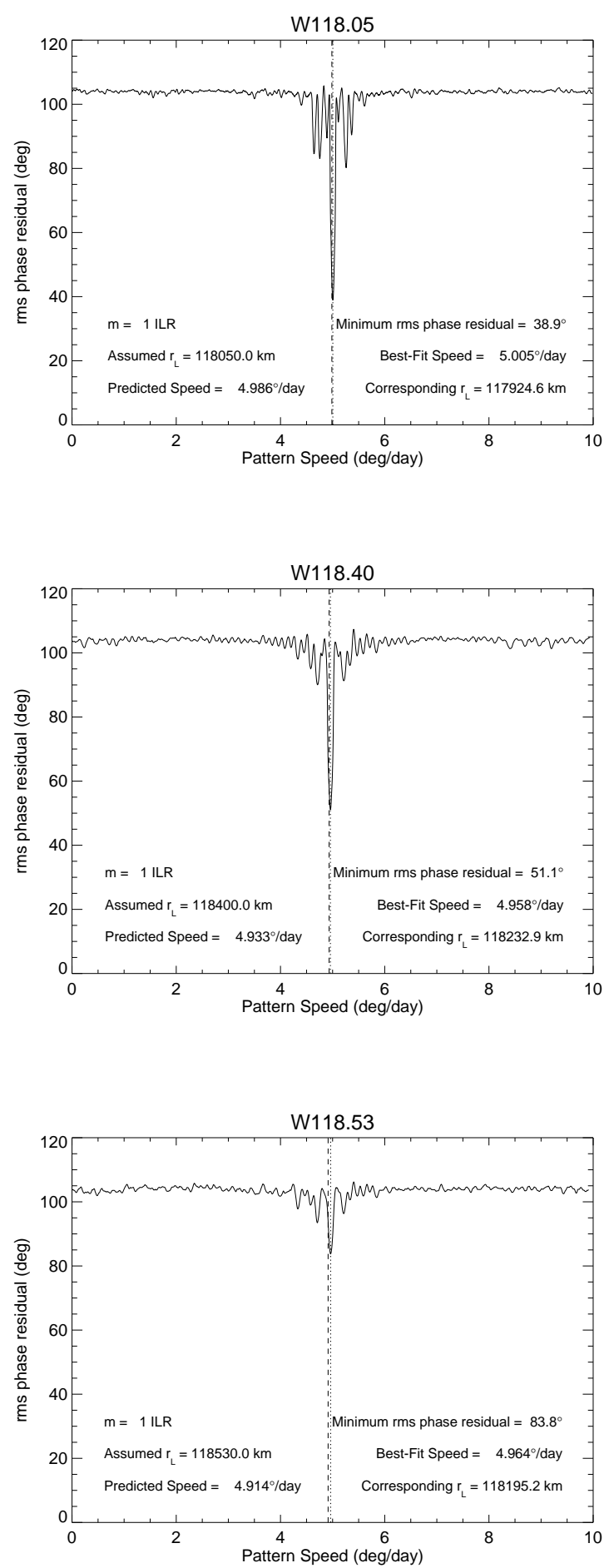

Figure 7. Pattern speed scans for waves W118.05, W118.40 and W118.53 for $m=1$, based on comparing the observed and predicted phase differences between pairs of occultations (see text for details). The data set includes all VIMS and RSS occultations, subject to signal-to-noise limitations. For each wave, the best-fitting pattern speed (indicated by the vertical dotted line) and corresponding resonant radius are listed, along with the minimum RMS phase residual. Also listed are the predicted values of $\Omega_{p}$ used to center our search region, indicated by the vertical dashed line. 
However, a careful inspection of Fig. 8 also shows that the picture becomes more complicated outside a radius of $\sim 118,080 \mathrm{~km}$, corresponding roughly to the radius of the weak Prometheus 9:7 density wave $\left(r_{L}=118,066 \mathrm{~km}\right)$. In this region we see what appear to be several partial segments of leading spirals (i.e., the radius of some crests seem to increase monotonically with increasing true anomaly). Moreover, in Fig. 3 we see in this same region a series of narrow features (e.g., the double-peak at $118,097 \mathrm{~km}$ and the single peaks at 118,109, 118,147 and 118,160 km) that seem to be axisymmetric rather than following the expected pattern for either type of spiral. One possible explanation, inspired by our results on the W120.20 wave described below, is that the $m=1$ trailing spiral is partially reflected at the inner edge of the Herschel gap and propagates back towards the inner edge of Band 1 as a leading spiral. The result may look somewhat like an $m=1$ standing wave, for which the crests move neither inward nor outward with time, but simply oscillate in place. Provocatively, Lehmann et al. (2019) (cf. Fig. 5) show a very similar result for a hydrodynamical simulation of a non-linear density wave partially reflected at a sharp discontinuity in the background optical depth.

\section{2. $W 118.40$ and $W 118.53$}

Based on Figs. 6 and 7 above, the waves in Band 2 appear to be fit best by a single ILR-type density wave with $m=1$. In this case, $\Omega_{p}=4.958 \pm 0.005^{\circ} \mathrm{d}^{-1}$ and the resonant radius is $118,230 \pm 35 \mathrm{~km}$. This places the likely source of the wave within or very near the Herschel ringlet, which has a mean radius of $118,249 \mathrm{~km}$ and an average width of $32 \mathrm{~km}$ (French et al. 2016a). The next-nearest feature is the inner edge of the Herschel gap at 118,188 km. Here, the series of occultation profiles in Fig. 4 and the wavelet spectra in Fig. 6 both show a clear trend of radial wavelength decreasing with increasing radius, as expected for an ILR-type density wave. Despite the interruption of this wave by a narrow, circular feature at $\sim 118,465 \mathrm{~km}$ due to

the density wave driven by the weak Pan 6:5 ILR $\left(r_{L}=118,453 \mathrm{~km}\right)$, the W118.40 and W118.53 waves seem to form part of a single wavetrain, with eight crests interior to $118,465 \mathrm{~km}$, and $\sim 15$ exterior to this radius. But based on the larger RMS phase residuals seen for W118.53, we conclude that the wave is less well-organized in this region than it is closer to the resonant radius. In this respect it may be similar to the W118.05 wave, though we have not been able to find any evidence of a reflected wave here.

\subsection{A regional view of the inner Cassini Division}

Figure 9 summarizes all of the measured $m=1$ pattern speeds for the inner Cassini Division, drawing on the results of this paper and those of French et al. (2016a). Sharp edges of gaps and ringlets are indicated by filled circles, while the pattern 


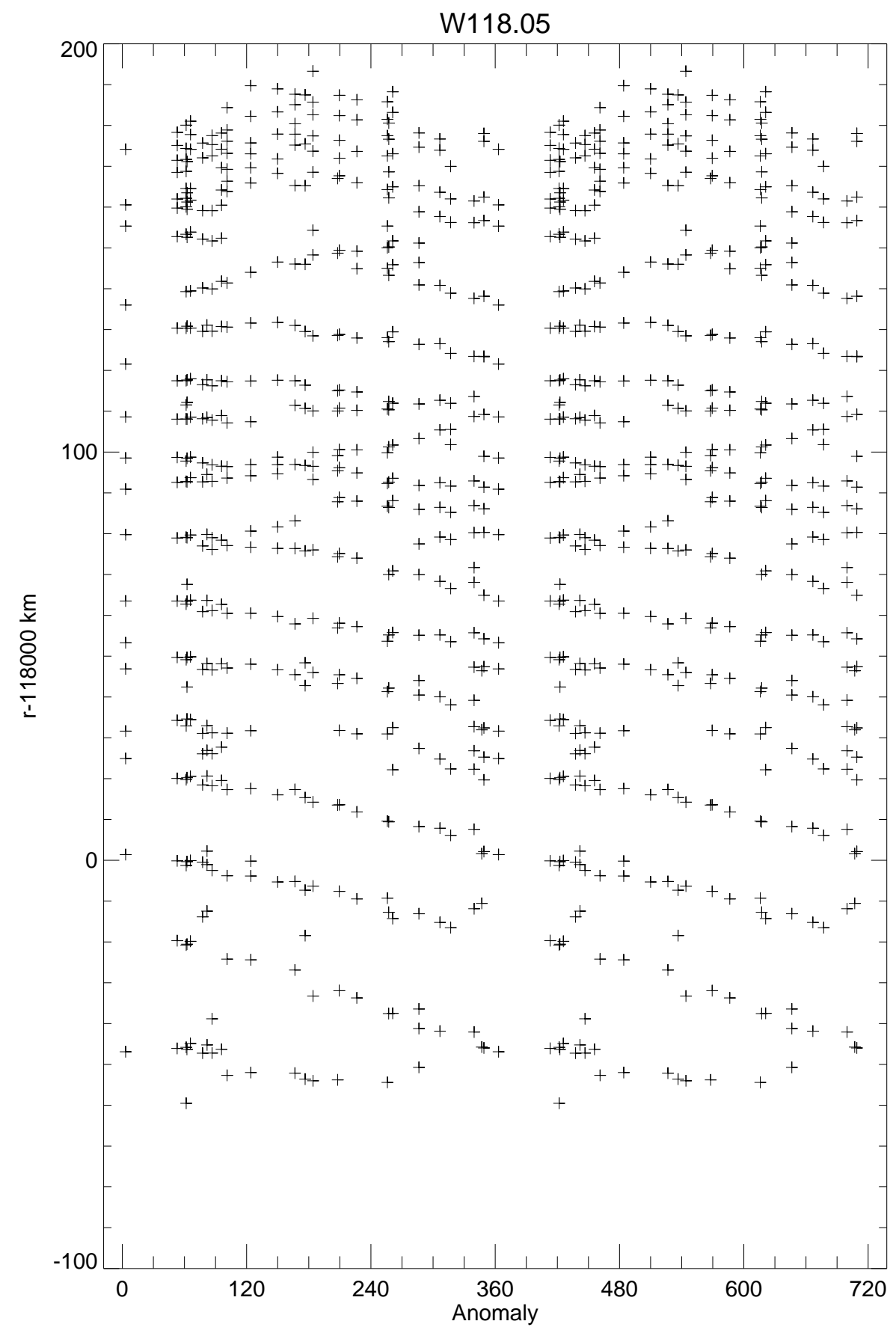

Figure 8. Successive wave crest locations for RSS observations of wave W118.05 in Band 1 , as functions of true anomaly $f$. Series of points for which the radius decreases with increasing $f$ correspond to a trailing spiral pattern, whereas points for which the radius increases with increasing $f$ correspond to a leading spiral. Both types of spiral are seen here, but the trailing spiral is dominant interior to $118,090 \mathrm{~km}$. Note that two full cycles of true anomaly are shown, in order to demonstrate that, in most cases, each arm of the trailing spiral pattern connects smoothly to the next one further inwards, as expected for an $m=1$ spiral. A total of 11 or 12 circuits of the trailing spiral are visible here, beginning at a radius of $\sim 117,950 \mathrm{~km}$. The inferred resonant radius is $117,920 \pm 35 \mathrm{~km}$. 
speeds for the waves in Bands 1 and 2 are shown as open circles, with error bars. The diagonal line is the predicted apsidal precession rate $\dot{\varpi}_{\mathrm{sec}}$, based on Saturn's zonal gravity harmonics (Jacobson et al. 2008). As noted by French et al. (2016a), almost all pattern speeds in this part of the rings are slightly higher than predicted, probably because of the unmodeled effect of the nearby and massive $\mathrm{B}$ ring. Particularly noticeable in this respect are the anomalously fast rates exhibited by the outer edges of the B ring itself (Nicholson et al. 2014a) and the Huygens gap, and the inner edges of the Herschel and Kuiper gaps, all of which are currently unexplained.

For each of the $m=1$ waves studied here, a horizontal dotted line connects the measured pattern speed to the resonant radius, i.e., the radius where $\dot{\varpi}_{\mathrm{sec}}=\Omega_{p}$. These lines reinforce the similarities in pattern speed (or apsidal precession rate) of the W118.05 wave and the Strange ringlet, and of the W118.40/W118.53 wave and both edges of the Herschel ringlet. (Although the inferred resonant radius for the W118.05 wave is also close to the outer edge of the Huygens gap, as noted above, the pattern speed matches that of the Strange ringlet much more closely. A similar statement applies to the W118.40/W118.53 wave and the inner edge of the Herschel gap.) Both waves therefore exhibit pattern speeds that are significantly faster than the local value of $\dot{\varpi}_{\mathrm{sec}}$, but which match that of a nearby eccentric ringlet. Based on these facts, and on the close similarities between the two waves, we suggest that the source of the waves is likely to be the Strange (or R6) ringlet in the case of wave W118.05 in Band 1 and the Herschel ringlet in the case of wave W118.40/W118.53 in Band 2. More specifically, we propose that the ringlet eccentricities first drive forced $m=1$ perturbations in the ring material adjacent to the nearby outer gap edges, which in turn generate outward-propagating $m=1$ density waves. A very similar situation was studied by Hahn (2008), but in that study the eccentric ringlet was replaced by an eccentric moonlet. ${ }^{5}$

There are, however, potential difficulties with this simple picture, chief of which is that the observed apsidal precession rates of the intervening gap edges - i.e., the outer edges of the Huygens and Herschel gaps - differ measurably from those of the ringlets and the density waves (see Fig. 9). Second, the inner and outer eccentric edges of the Herschel ringlet are significantly misaligned, with relatively small eccentricities (ae $\sim 1-2 \mathrm{~km}$ ), raising the question of whether it could really drive an $m=1$ wave, although some degree of misalignment is expected due to the viscosity of the ring (Borderies et al. 1983). A third puzzle is why the wave in Band 1 is apparently driven by the Strange ringlet rather than by the Huygens ringlet, even though the latter is both more eccentric ( $a e=29 \mathrm{~km}$ vs $7.5 \mathrm{~km}$; see Table 2 of French et al.

\footnotetext{
${ }^{5}$ These circumstances are more similar than they might first appear, as the theory for a moonlet driving an $m=1$ density wave begins with averaging the gravitational effect of the moonlet around its eccentric orbit, thereby effectively replacing it by an eccentric ringlet. As in the present work, the wave predicted by Hahn (2008) is a trailing $m=1$ spiral that propagated outwards from the gap with a pattern speed equal to the apsidal precession rate of the moonlet.
} 


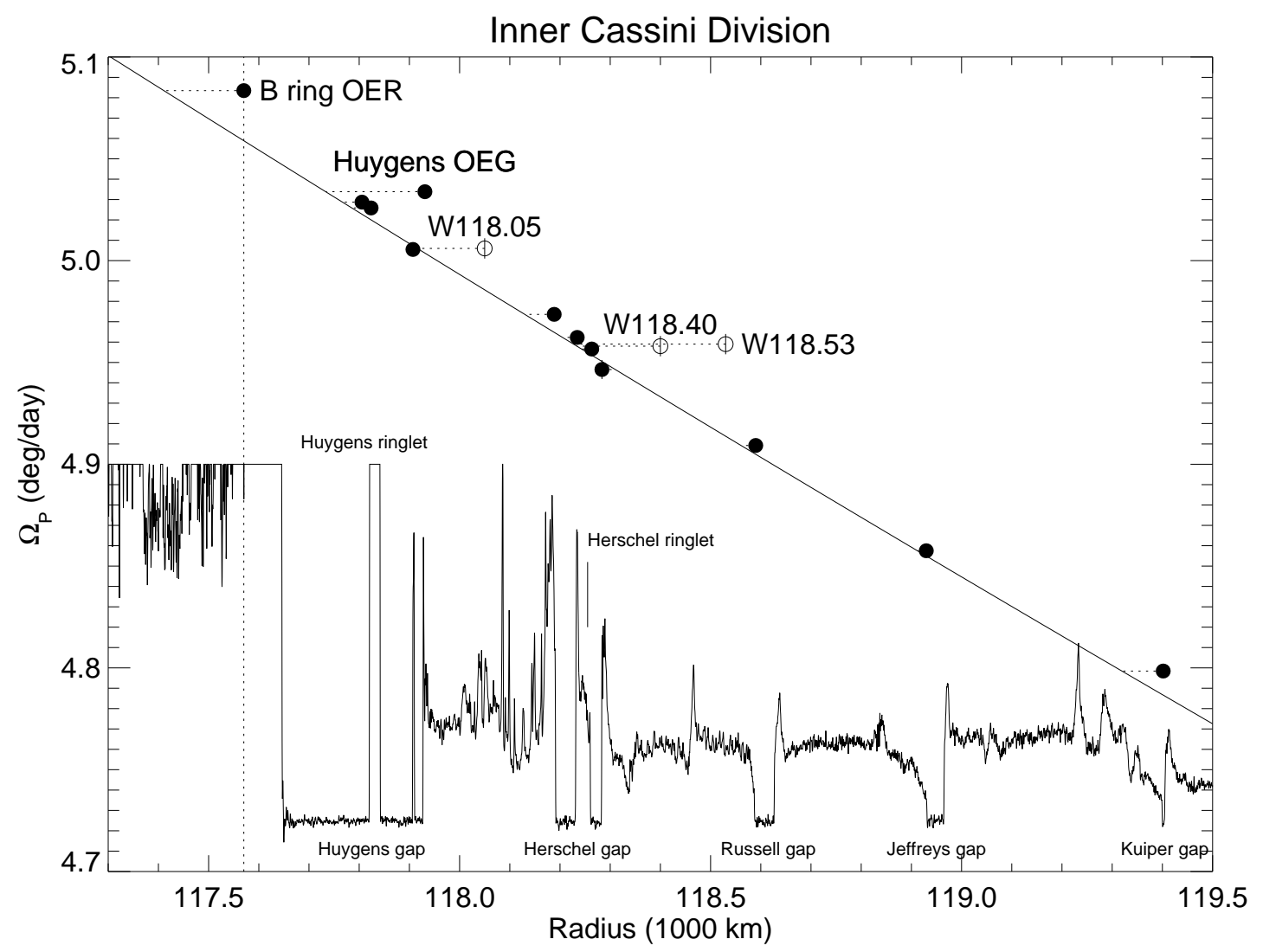

Figure 9. Regional $m=1$ pattern speeds in the inner Cassini Division. Filled circles denote gap and ringlet edges from French et al. (2016a) while open circles show the waves discussed in this paper. The diagonal line shows the apsidal precession rate $\dot{\varpi}_{\text {sec }}$ calculated from Saturn's zonal gravity harmonics. Horizontal dotted lines connect the measured pattern speeds for the gaps or ringlets and waves to their corresponding resonant radii. The vertical dotted line at $117570.12 \mathrm{~km}$ marks the mean radius of the outer edge of the $\mathrm{B}$ ring, from Table 4 of Nicholson et al. (2014a).

(2016a)) and probably much more massive than the former. We will return to these vexing questions in Section 7.4 below.

\section{POSSIBLE VERTICAL STRUCTURE IN BANDS 1 AND 2}

\subsection{RSS profiles}

Further investigation provides still more evidence that the picture outlined above is not the whole story. In compiling the galleries of profiles in Figs. 3 and 4 we observed that, although the RSS occultations all have very similar signal-to-noise levels (unlike the stellar occultations, for which the stellar flux and ring opening angle vary significantly between target stars), the prominence of the waves varies noticeably between the observations. Closer inspection shows that the waves generally become more prominent as the opening angle of the rings becomes smaller, suggesting a vertical (i.e., out of the mean ring plane) component to the waves. 
This can be tested by sorting the RSS profiles by the angle $B_{\text {eff }}$, first introduced by Gresh et al. (1986) and Nicholson et al. (1990) to characterize the effect of vertical ring structure on RSS occultation profiles. Simple geometry shows that the apparent radial displacement $\Delta r$ of a vertically-offset feature in the rings is given by $\Delta r=$ $-z / \tan B_{\text {eff }}$, where

$$
\tan B_{\text {eff }}=\frac{\tan B_{\oplus}}{\cos \left(\lambda-\lambda_{\oplus}\right)} .
$$

Here, $\lambda$ and $z$ are the longitude and vertical height of the feature, $B_{\oplus}$ is the elevation of the Earth above the ring plane, and $\lambda_{\oplus}$ is the saturnicentric longitude of the Earth. (A similar equation applies to stellar occultations, with $B_{\odot}$ and $\lambda_{\oplus}$ replaced by $B_{*}$ and $\lambda_{*}$, respectively (Jerousek et al. 2011; Nicholson and Hedman 2016).) In Figs. 10 and 11 we replot the same galleries of RSS profiles for the W118.05 and W118.40/118.53 waves, but now sorted in order of increasing $\left|B_{\text {eff }}\right|$. In Fig. 11 we see that the W118.40/118.53 waves are most prominent for small values of $B_{\text {eff }}$ and much less obvious for $\left|B_{\text {eff }}\right|>20^{\circ}$. In the W118.05 region the waves between 118,000 and $118,060 \mathrm{~km}$ are also more clearly seen at $\left|B_{\text {eff }}\right|<20^{\circ}$ (Fig. 10). This seems to confirm our suspicion that there may be an out-of-plane component to both waves.

As supporting evidence, we note that not only are the Strange and Herschel ringlets known to be eccentric, they also both exhibit small inclinations with respect to the mean ring plane (French et al. 2016a). It is thus conceivable that they might drive bending waves as well as density waves in the adjacent ring material, as envisioned by Hahn (2007) for an inclined satellite. As in his parallel investigation of an $m=1$ density wave driven by a nearby eccentric moonlet (Hahn 2008), the theory for a moonlet driving an $m=1$ bending wave begins with averaging the gravitational effect of the moonlet around its inclined orbit, thereby effectively replacing it by a circular but inclined ringlet. As discussed below, the bending wave modeled by Hahn (2007) is a leading $m=1$ spiral that propagates outwards from the gap with a negative pattern speed equal to the nodal regression rate of the moonlet. Interior to the gap containing the moonlet, the bending wave is evanescent, so no similar wave is to be expected.

\subsection{Bending waves?}

These considerations raise the possibility that the waves in the inner Cassini Division have some vertical component, or even that they might be bending waves rather than density waves. But the latter possibility immediately leads to additional complications. Most bending waves - at least those generated at Inner Vertical Resonances (IVRs) with external satellites such as the well-studied Mimas 5:3 bending wave in the A ring (Shu et al. 1983; Gresh et al. 1986) — propagate inwards, whereas we have seen above that the waves in question seem to propagate outwards. However, it turns out that bending waves with $m=1$ are peculiar in several respects, as first observed 


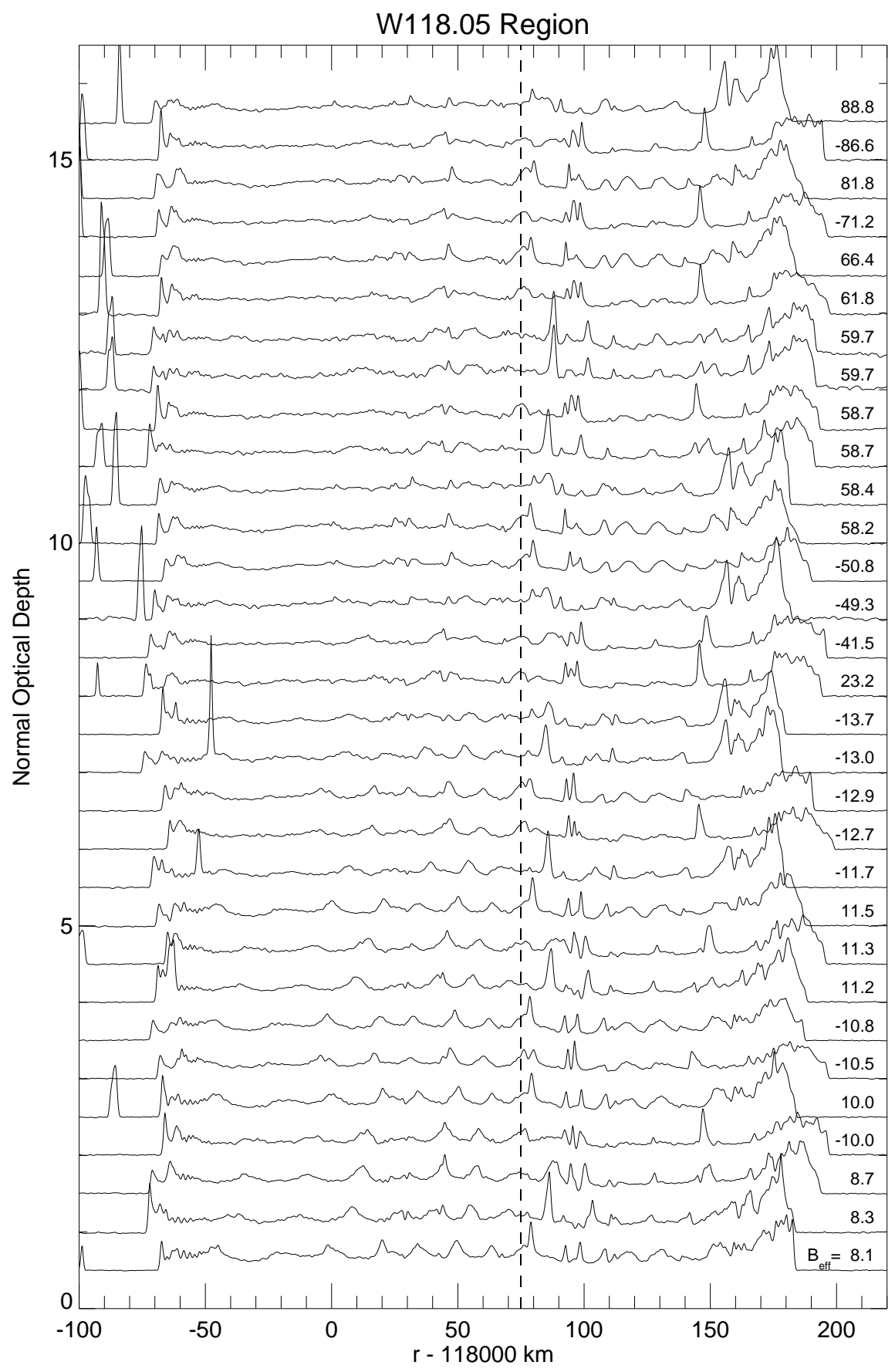

Figure 10. Profiles of wave region W118.05 in Band 1, sorted by the absolute value of $B_{\text {eff }}$. The data are the same as the RSS occultation profiles shown in Fig. 3. The vertical dashed line marks the Prometheus 9:7 ILR.

by Rosen and Lissauer (1988). Not only do they have unusually long wavelengths, like $m=1$ density waves, but their pattern speeds are negative. To see this, note that the analogous expression to Eq. (3) for a bending wave is:

$$
m \Omega_{p}=(m-1) n+\dot{\Omega}_{\mathrm{sec}}
$$




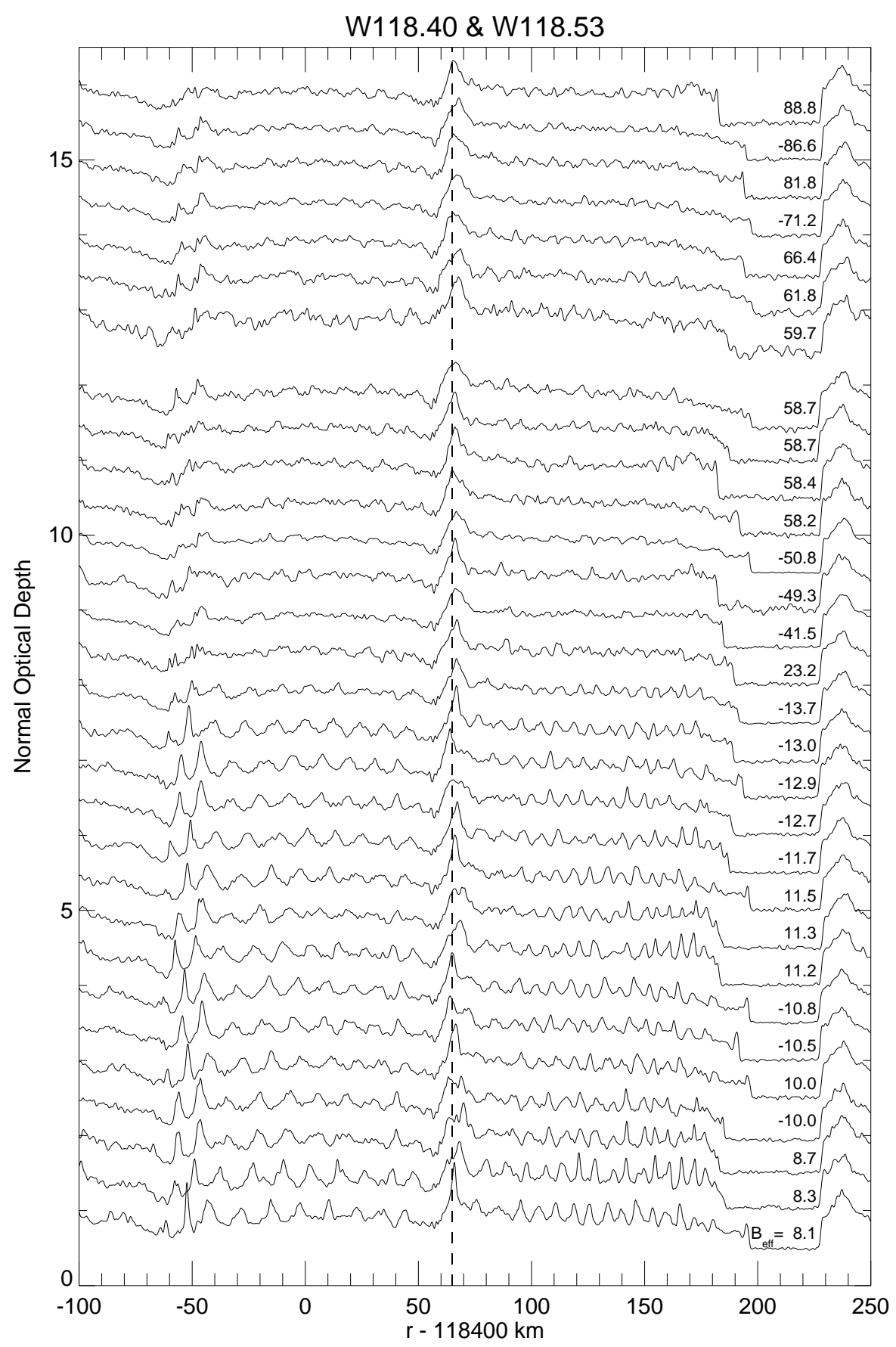

Figure 11. Profiles of waves W118.40 and W118.53 in Band 2, sorted by the absolute value of $B_{\text {eff. }}$ The data are the same as the RSS occultation profiles shown in Fig. 4 . The vertical dashed line marks the Pan 6:5 ILR.

where $\dot{\Omega}_{\text {sec }}$ is the nodal regression rate due to secular perturbations. For bending waves with $m=1$, we have $\Omega_{p}=\dot{\Omega}_{\text {sec }}$, which is negative for orbits about an oblate planet such a Saturn. In fact, $\dot{\Omega}_{\mathrm{sec}} \simeq-\dot{\varpi}_{\mathrm{sec}}$. There are two known $m=1$ bending waves in Saturn's rings, driven by the distant satellites Titan and Iapetus (Rosen and Lissauer 1988; Tiscareno et al. 2013; Nicholson and Hedman 2016). For each of these waves, 
the pattern speed is indeed observed to be negative with $\Omega_{p} \simeq-n_{s}$, where $n_{s}$ is the satellite's mean motion. As a result, these satellite-driven bending waves actually propagate outwards. Another unusual feature of $m=1$ bending waves driven by IVRs is that they take the form of a leading spiral, rather than the more common trailing geometry (Rosen and Lissauer 1988; Nicholson and Hedman 2016). Similar results were obtained by Hahn (2007) in his study of $m=1$ bending waves driven by a nearby inclined moonlet.

Thus, a satellite-driven (or perhaps ringlet-driven) outward-propagating $m=1$ bending wave does indeed appear to be theoretically possible, albeit with a negative pattern speed and the instantaneous form of a leading spiral. If this were the case, however, we would expect to see a systematic leading or trailing pattern in the radial locations of individual wave crests, when plotted as a function of ascending node $\Omega$. Figure 12 reveals no such pattern for W118.05, arguing against this possibility for the wave in Band 1.

We can also test the possibility that the $m=1$ waves in Bands 1 and/or 2 have a bending wave component by re-running the phase-matching wavelet analysis with the pattern speed given by Eq. (12). A practical complication is that the sign of the apparent radial displacement associated with a vertical wave changes with the sign of $B_{\text {eff }}$, as given by Eq. (11), and thus with the signs of both $B_{\oplus}$ and $\cos \left(\lambda-\lambda_{\oplus}\right)$. This introduces additional observer-dependent $180^{\circ}$ shifts into $\phi_{\lambda, t}$ beyond those associated with the wave itself that must be taken into account when modeling or correcting the wavelet phases. We have previously developed a simple but approximate method for adjusting the observed wavelet phases for bending waves, as described by French et al. (2019), that involves adding $180^{\circ}$ to $\phi_{j}$ whenever $B_{\text {eff }}<0$. The results are shown in Fig. 13, in the same format as Fig. 6.

Focussing on the third, fourth and fifth panels, where the phase correction is applied, we do not see strong evidence for $m=1$ vertical structure in either Band 1 or Band 2 . The strongest signal is seen at a radius of $117,700-118,000 \mathrm{~km}$ and $k \simeq 0.1 \mathrm{~km}^{-1}$. This appears to be due to the Strange ringlet, which is known to have a substantial inclination of $a \sin i=7.4 \mathrm{~km}$ and $\dot{\Omega}=-4.976^{\circ} \mathrm{d}^{-1}$ (French et al. 2016a). The only sign of a possible bending wave is a rather weak signal in Band 2 with $k$ increasing outward from $\sim 0.5 \mathrm{~km}^{-1}$ to $\sim 1.0 \mathrm{~km}^{-1}\left(12>\lambda_{r}>6 \mathrm{~km}\right)$.

\subsection{ISS images}

One might ask whether there is any other evidence for vertical structure in Bands 1 and 2. Surprisingly, this seems to be the case. A search for high-resolution Cassini images of the inner Cassini Division, using the OPUS tool on the PDS Ring-Moon Systems Node, identified several dozen images with a projected ring plane radial res- 
W118.05

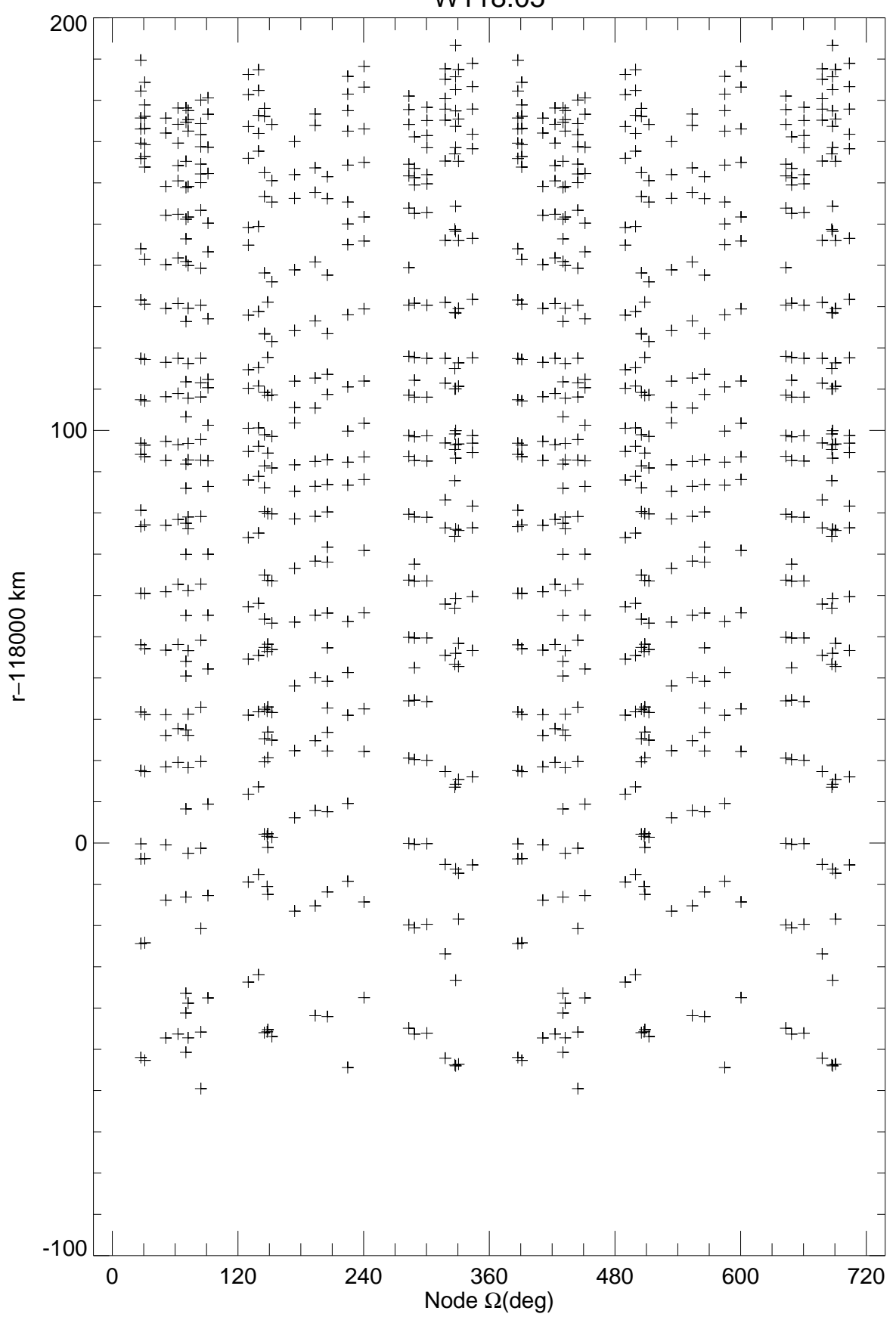

Figure 12. Successive wave crest locations for RSS observations of wave W118.05 in Band 1 as in Fig. 8, but plotted as a function of ascending node $\Omega$. There is no clear organized behavior to indicate that this is a bending wave.

olution below $2 \mathrm{~km} /$ pixel, some of which were obtained near the saturnian equinox on 11 August 2009, when the sun angle on the rings was extremely low. This illumination geometry is especially sensitive to vertical structure such as bending waves, with the ring brightening whenever its local surface is tilted towards the sun. We have analyzed five images that are particularly revealing, with parameters listed at 


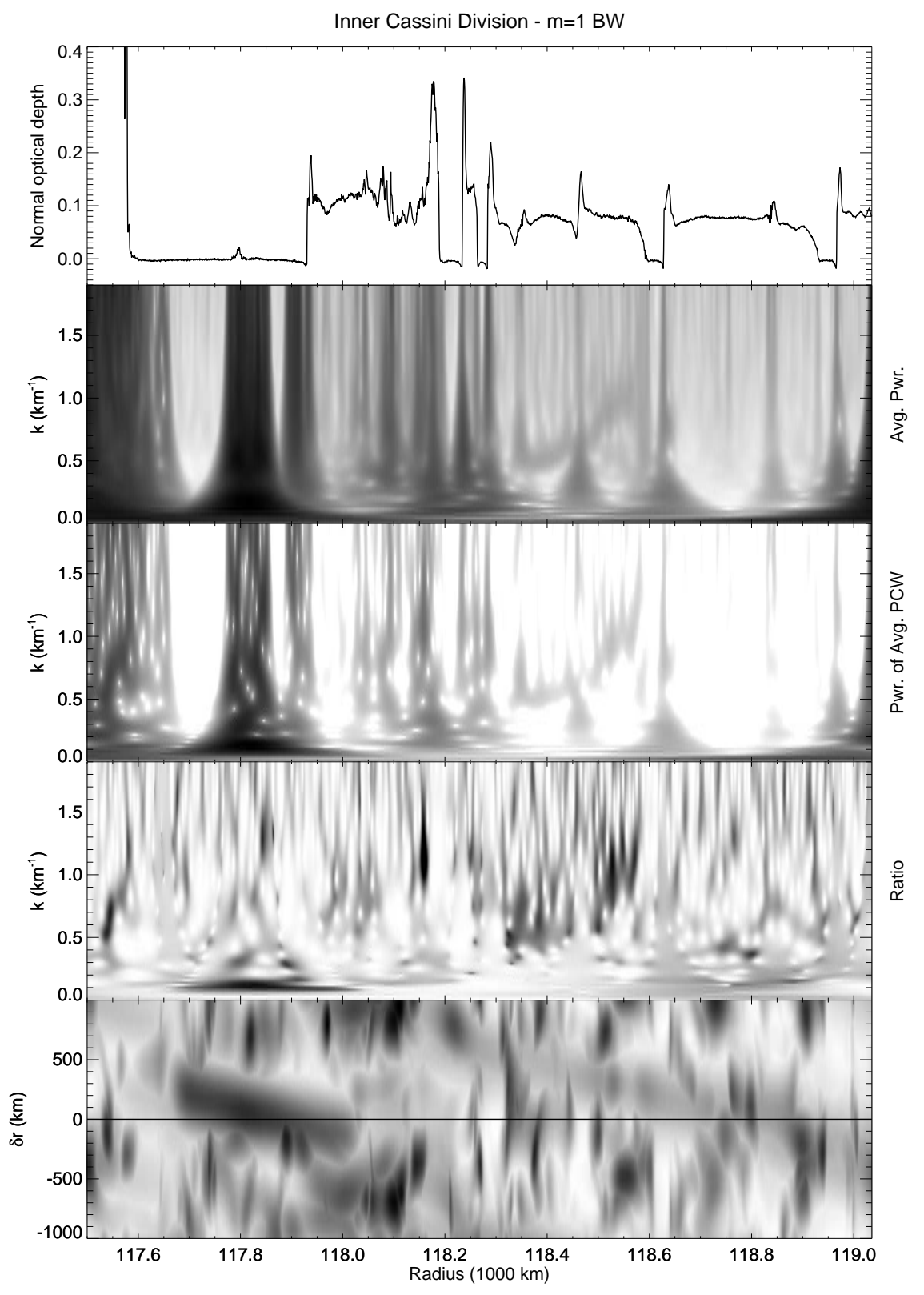

Figure 13. Phase-corrected wavelet analysis for Bands 1 and 2, assuming that the structure is due to bending waves with $m=1$ and $\Omega_{p} \simeq \dot{\Omega}_{\mathrm{sec}}$. The format is the same as that of Fig. 6, except that the 6th panel is omitted here. No clear bending wave signals are seen, except the power at low wavenumbers between 117,700 and $117,950 \mathrm{~km}$, which is likely due to the inclined Strange (R6) ringlet.

the top of Table 1. All are of the sunlit (i.e., southern) side of the rings. Just as for the RSS occultations, the sensitivity of images to vertical structure is conveniently parameterized by the effective elevation angle $B_{\text {eff }}$, given by Eq. (11), but in this case $B_{\oplus}$ and $\lambda_{\oplus}$ are replaced by the equivalent solar elevation $B_{\odot}$ and longitude $\lambda_{\odot}$. In 
Table 1. Cassini ISS NAC images

\begin{tabular}{|c|c|c|c|c|c|c|c|}
\hline Image & Observation name & Midtime & $B_{\mathrm{sc}}(\operatorname{deg})$ & $B_{\odot}(\operatorname{deg})$ & $B_{\text {eff }}(\operatorname{deg})$ & $H(\operatorname{deg})$ & $\mathrm{km} / \mathrm{px}^{(a)}$ \\
\hline \multicolumn{8}{|l|}{ Figs. 14 and 15: } \\
\hline N1495327885 & ISS_008RI_RDHRCOMP001 & 2005-141Т00:24:09 & -14.70 & -21.64 & 52.51 & 287.72 & 1.75 \\
\hline N1595342033 & ISS_077RI_RDHRCOMP001 & 2008-203T13:55:42 & -66.08 & -5.95 & 6.88 & 329.66 & 1.10 \\
\hline N1625956787 & ISS_114RI_EQXSHADOW003 & 2009-191T21:57:58 & -31.37 & -0.48 & -0.48 & 177.19 & 1.75 \\
\hline N1627303569 & ISS_115RI_AZSCANLIT001 & 2009-207T12:04:10 & -50.38 & -0.24 & 0.29 & 35.17 & 1.44 \\
\hline N1627307734 & ISS_115RI_AZSCANLIT001 & 2009-207T13:13:35 & -48.81 & -0.24 & 0.35 & 46.89 & 1.27 \\
\hline \multicolumn{8}{|l|}{ Fig. 21: } \\
\hline N1560311811 & ISS_046RI_RDHRESSCN001 & 2007-163Т03:22:40 & -33.77 & -12.06 & 89.71 & 89.94 & 0.99 \\
\hline N1870073325 & ISS_268RI_HRRADSCNL001 & 2017-095Т07:40:28 & 74.57 & 26.72 & 26.74 & 182.30 & 0.79 \\
\hline
\end{tabular}

${ }^{(a)}$ Projected ring plane radial resolution

terms of the hour angle used by the PDS, defined as $H=\lambda-\lambda_{\odot}+180^{\circ}$, we have

$$
\tan B_{\text {eff }}=-\frac{\tan B_{\odot}}{\cos H}
$$

Images with small values of $B_{\text {eff }}\left(i . e\right.$. , those with low $B_{\odot}$ and targets near the subsolar or antisolar directions, so that $|\cos H|$ is close to 1$)$ will show alternate brightening and dimming across near-axisymmetric vertical ripples, while images with large values of $B_{\text {eff }}$ (i.e., those with high $B_{\odot}$ and/or targets near $90^{\circ}$ from the sub solar direction) are insensitive to such variations in the surface tilt.

After photometrically calibrating and then navigating each image (by correcting its nominal pointing using circular ring features with known radii), we reprojected them into radius-longitude coordinates at a common radial scale, as shown in Fig. 14. We then extracted a mean radial profile of brightness $(I / F)$ for each image, plotted in Fig. 15 in the same order as in Fig. 14. Vertical dashed lines in this figure show the predicted (apparent) radius for selected sharp-edged features, taking account of possible non-circularities and inclinations using the orbit models of French et al. (2016a). From left to right, these are the Huygens and Strange ringlets, the outer edge of the Huygens gap, the Herschel gap and its embedded ringlet, and the edges of the Russell and Jeffreys gaps. In most instances, the predicted radii match those seen in the image scans, providing confirmation of our geometric reconstructions. We briefly discuss each image in turn.

N1495327885: This image was taken in 2005 on day 141, with a relatively high solar elevation angle $B_{\odot}=-21.64^{\circ}$. The target point was on the right ansa, $\sim 70^{\circ}$ from the antisolar direction (hour angle $H=287.72^{\circ}$ ), a geometry that is quite insensitive to out-of-plane structure, as is indicated by value of $B_{\text {eff }}=52.51^{\circ}$. We see that all gaps and narrow ringlets are at their expected locations, with the only other notable structure being a series of weak oscillations in $I / F$ in Band 1, corresponding to wave 


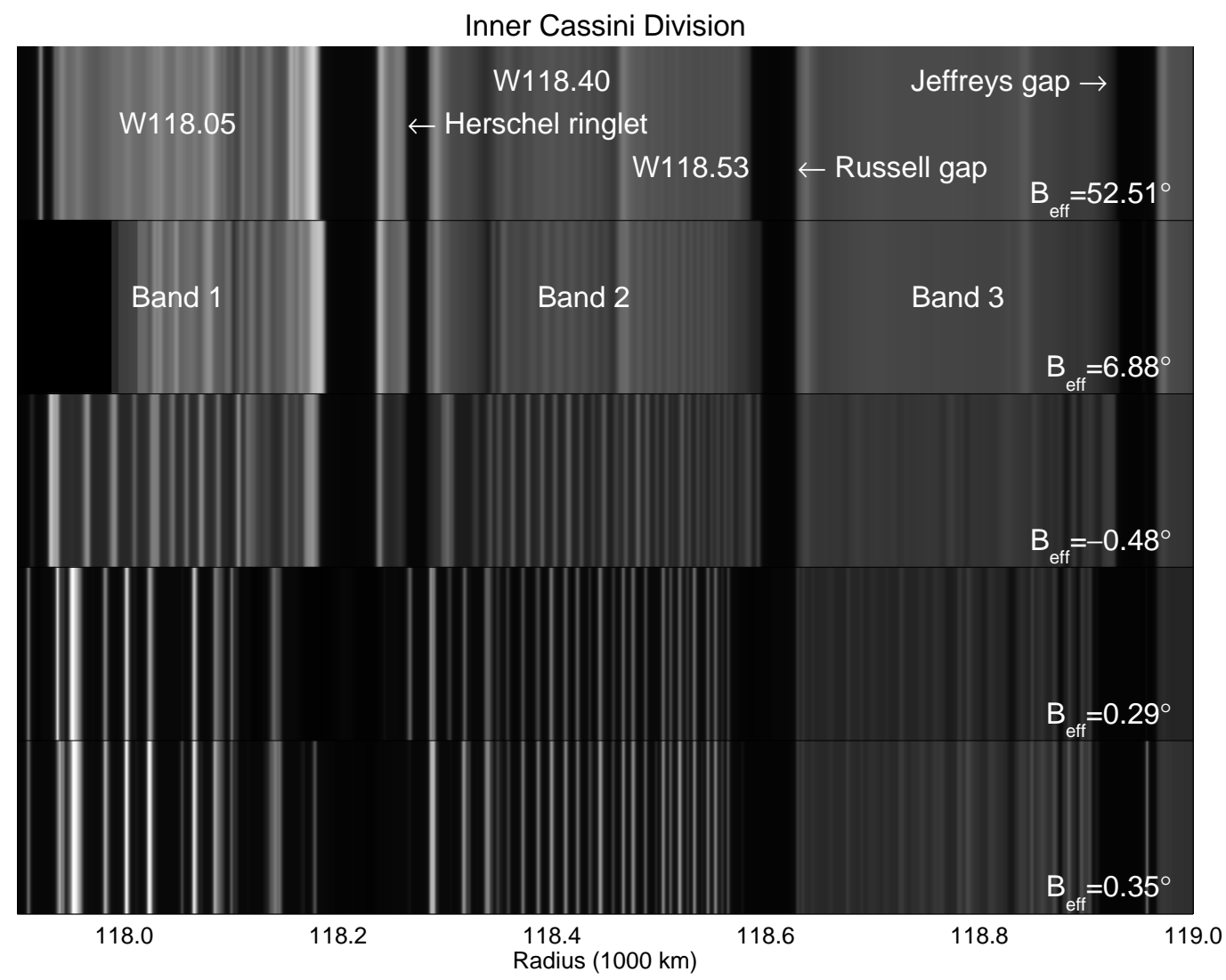

Figure 14. Five Cassini ISS narrow angle camera images of the inner Cassini Division, reprojected at a uniform scale into radius and longitude. The effective ring opening angle $B_{\text {eff }}$ is labeled at right for each image; see Table 1 for a list of image numbers and additional geometric parameters. Visible from left to right are the outer Huygens gap edge (except in the 2nd image), the Herschel gap and ringlet, the Russell gap and the Jeffreys gap. Bounded by these gaps are Bands 1, 2 and 3 of Flynn and Cuzzi (1989). The wave structures in Bands and 2 are especially prominent in the lower three images (see text).

W118.05, and even weaker oscillations in Band 2, corresponding to W118.40 and W118.53.

N1595342033: This image was taken in 2008 on day 203, with a much lower solar elevation angle $B_{\odot}=-5.95^{\circ}$. The target point was near the edge of the shadow cast by Saturn across the rings, at hour angle $H=329.66^{\circ}$, making the illumination geometry much more sensitive to out-of-plane structure, with $B_{\text {eff }}=6.88^{\circ}$. All visible gaps and narrow ringlets are again at their expected locations, but the wavelike structure in Bands 1 and 2 is now much more visible than in the first image, as we found above for the lower-inclination RSS occultation profiles. The average $I / F$ is $\sim 0.006$.

N1625956787: This image was taken in 2009 on day 191, only 1 month before the saturnian equinox on day 223 , with a solar elevation angle of $-0.48^{\circ}$ and a target point close to local noon $\left(H=177.19^{\circ}\right)$, so that $B_{\text {eff }}=-0.48^{\circ}$. Again, all of the 


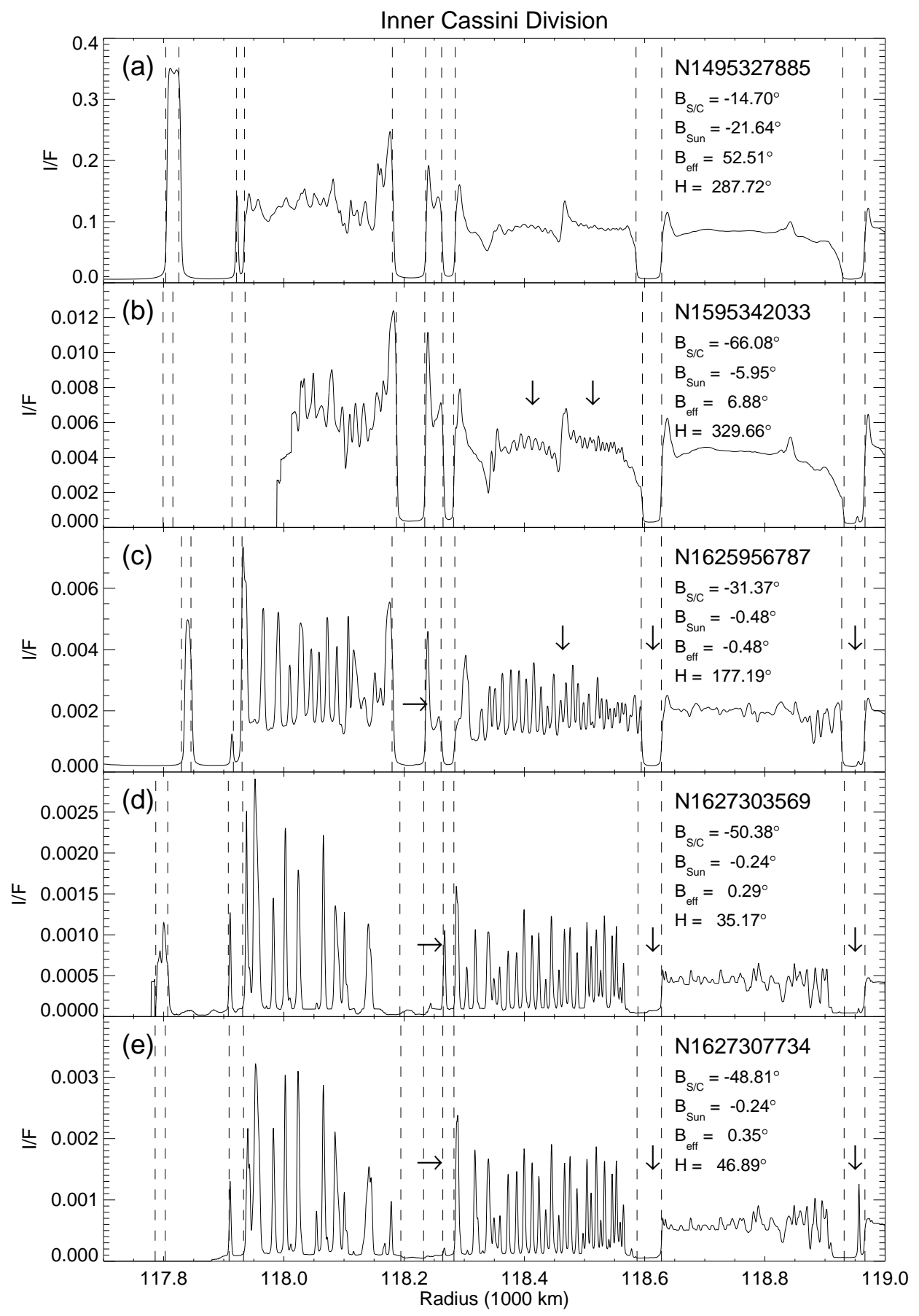

Figure 15. Radial scans of the ISS NAC images shown in Fig. 14. See Table 1 for a list of image numbers and geometric parameters. Visible from left to right are the Huygens ringlet and outer gap edge, the Herschel gap and ringlet, the Russell gap and the Jeffreys gap. Vertical dashed lines correspond to the predicted apparent radius of sharp-edged features, taking account of their possibly non-circular and/or inclined orbital shapes. The wave structures W118.05, W118.40 and W118.53 are especially prominent in the lower three profiles (see text). Arrows highlight the changing features noted in the text. 
gaps and ringlets are recognizable, though the average $I / F$ is only $\sim 0.0025$. The wavelike structure in Bands 1 and 2 is now much more prominent, and several wavelike oscillations are also seen in Band 3, immediately interior to the Jeffreys gap, at $\sim 118,840 \mathrm{~km}$. The feature normally seen near the center of Band 2 due to the Pan 6:5 density wave has disappeared, and the Herschel ringlet looks notably asymmetric, being much brighter on the inner edge. We note that French et al. (2016a) found evidence for non-zero inclinations on the inner and outer edges of this ringlet with amplitudes $a \sin i=1.5$ and $2.1 \mathrm{~km}$, respectively. A check of the ring and image geometry shows that these elevations are sufficient to place the outer edge of the ringlet within the shadow cast by the outer edge of the gap, but not the inner edge (see Appendix A).

N1627303569 and N1627307734: Both of these images were taken in 2009 on day 207, just 16 days before the equinox, with solar elevation angle $B_{\odot}=-0.24^{\circ}$ and targeted close to the shadow edge, at hour angles $H$ of $35.17^{\circ}$ and $46.89^{\circ}$, resulting in $B_{\text {eff }}=0.29^{\circ}$ and $0.35^{\circ}$, respectively. Several aspects of this pair of images are quite unusual. First, we note that the wave structure in Bands 1 and 2 is now so strong that the $I / F$ is virtually zero between the wave crests. This is strong support for the suggestion above that all three of waves W118.05, W118.40 and W118.53 exhibit some sort of vertical structure, perhaps even enough to cast shadows across the troughs of the waves. Secondly, we observe that the Herschel ringlet (predicted to appear at $118,233-112265 \mathrm{~km}$ ) has now almost completely disappeared, except for a very narrow spike at its outer edge in image N1627303569. In this case, a check of the ring and image geometry confirms that this ringlet falls fully within the shadow cast by the inner edge of the gap (see Appendix A).

How might we reconcile clear evidence for vertical structure associated with the waves in Bands 1 and 2 in both the RSS occultation profiles in Figs. 10 and 11 and in several ISS images with unusually low values of $B_{\text {eff }}$ with an apparent absence of the wavelet signature expected for a coherent bending wave with $\Omega_{p}=\dot{\Omega}_{\mathrm{sec}}$ in Fig. 13 ? One possibility, suggested in theoretical work by Borderies et al. (1985) and by the 3D ring simulations of Lewis and Stewart (2000), is that material in the crests of density waves can splash above and below the ring plane due to strong radial compression. Such vertically-extended material would be in phase with the underlying density wave, and might well enhance the line-of-sight optical depth in wave crests for lowinclination occultations as well as provide increased surface area for light scattering at low values of $B_{\odot}$. At the same time, the pattern speed of the wave itself is that expected for an $m=1$ density wave, rather than for a bending wave, accounting for the absence of any strong signature in Fig. 13. Of course, similar splashing phenomena would also be expected in association with satellite-driven density waves, and might be revealed by careful studies of such waves in low- $B_{\text {eff }}$ radio and stellar occultations, or in images taken of the $\mathrm{A}$ ring at very low values of $B_{\odot}$. 
Finally, an examination of the Russell and Jeffreys gaps in the final pair of profiles in Fig. 15, normally centered at 118,609 and 118,948 km, shows that while their outer edges are in the correct locations their inner edges are both displaced inwards relative to their predicted radii by $20-25 \mathrm{~km}$. This greatly exceeds their measured eccentricities (which are included in the predicted radii in any event) and the direction of the solar illumination means that the inner edge casts a shadow towards the outer edge, rather than vice versa. The only plausible explanation seems to be a gradually increasing tilt of the inner gap edge until it exceeds $B_{\text {eff }}$, at which point the edge can shadow itself. This appears to be consistent with the vertical amplitudes for these edges found by French et al. (2016a) of $0.065 \mathrm{~km}$ for the Russell gap and $0.17 \mathrm{~km}$ for the Jeffreys gap (see Appendix A for details), although we are lacking the detailed knowledge of the vertical shape of the edges to prove this definitively.

\section{WAVE STRUCTURE IN THE OUTER CASSINI DIVISION}

Our first indication of wavelike structure in the outer Cassini Division came from inspecting a series of RSS occultation profiles of the region immediately outside the Laplace gap, as shown in Fig. 5 above. These profiles show a regular series of at least four peaks in optical depth between radii of 120,180 and 120,230 km, as well as hints of similar but weaker peaks down to $\sim 120,140 \mathrm{~km}$. Closer inspection, however, reveals that, unlike the waves in Bands 1 and 2, seen in Figs. 3 and 4, these peaks do not seem to progress regularly inwards with increasing true anomaly. Instead, one finds a series of profiles all with peaks at the same radii, and then an abrupt shift to a second series of profiles where the peaks and troughs in optical depth seem to be $180^{\circ}$ out of phase with the first set. This is more clearly seen in Fig. 16, where we show four profiles from one subset of the RSS occultation profiles and four profiles from the other. Vertical dashed lines indicate how the peaks in optical depth for the first set become the troughs in the second set.

The facts that (i) the appearance and especially the phases of the RSS profiles are well-organized when they are sorted by true anomaly, i.e., by $f=\lambda-\lambda_{0}-\dot{\varpi}_{\text {sec }}\left(t-t_{0}\right)$, and (ii) there appear to be just two changes of phase in the pattern as $f$ varies though $360^{\circ}$ both suggest that this is an ILR-type perturbation with $m=1$, i.e., one that satisfies Eq. (3), albeit an unusual one. We can test this hypothesis by carrying out the same phase-corrected wavelet analysis as was done for the waves in Bands 1 and 2 above. The results are shown in Fig. 17, where the multi-panel plot has the same format as Fig. 6 above. In the third and fourth panels, we do indeed see a strong signal of an $m=1$ wave, with a radial wavenumber increasing from $k \simeq 0.4 \mathrm{~km}^{-1}$ at $120,150 \mathrm{~km}$ to $\sim 1.4 \mathrm{~km}^{-1}$ at $120,230 \mathrm{~km}$ (i.e., wavelengths decreasing from $16 \mathrm{~km}$ to $4.5 \mathrm{~km}$, consistent with Fig. 5). The average best-fitting pattern speed is $4.717^{\circ} \mathrm{d}^{-1}$, varying by $\pm 0.005^{\circ} \mathrm{d}^{-1}$ over the extent of the wave, corresponding to a resonance 


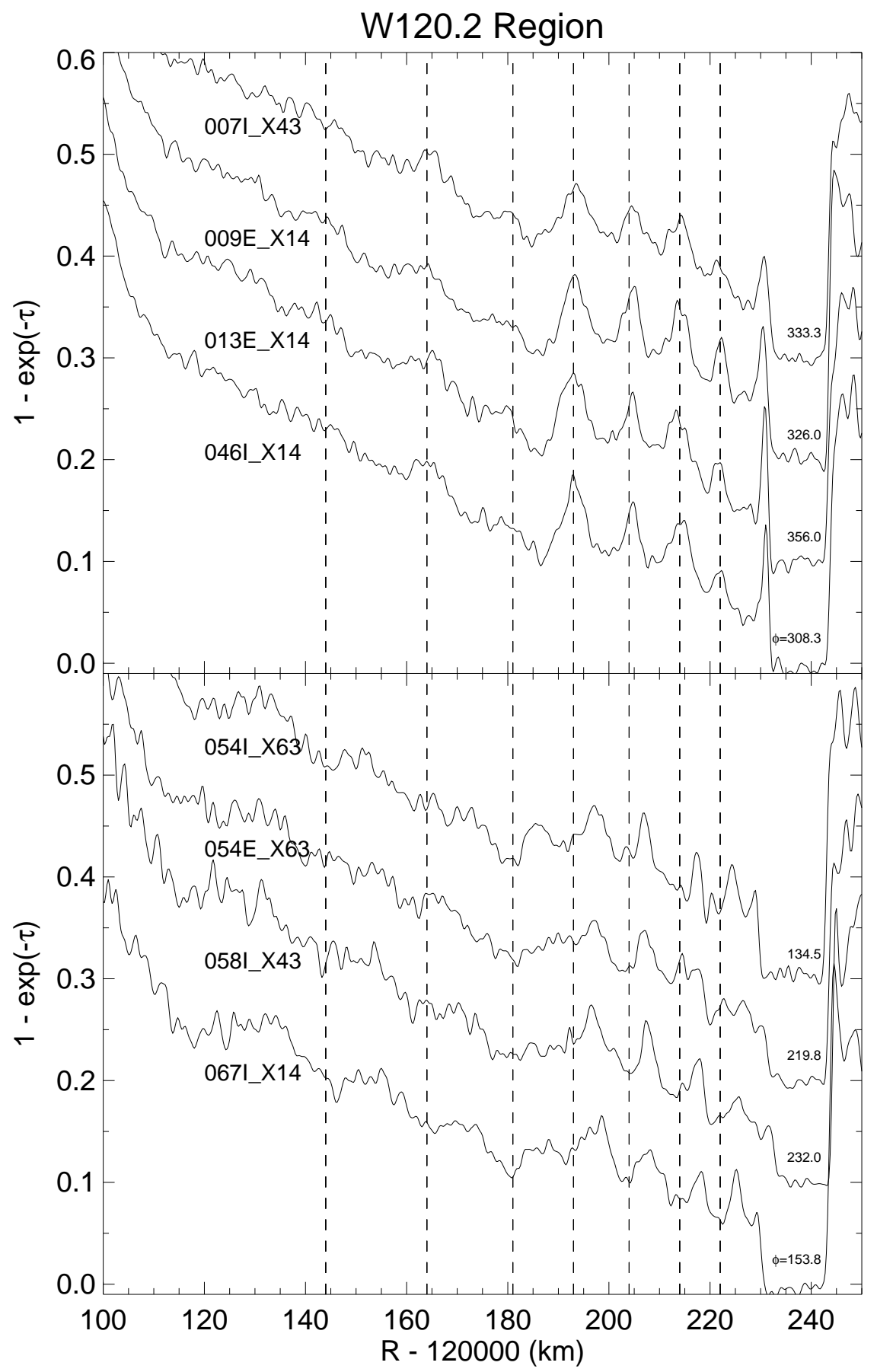

Figure 16. A subset of RSS occultation profiles of the W120.2 region, selected to illustrate the bimodal character of the wave crests and troughs. The profiles are labeled by Cassini rev number, I or E for ingress or egress, and by the RSS wavelength band (X, in all cases shown here) and NASA Deep Space Net station number. Vertical dashed lines indicate the approximate locations of optical depth maxima in the top set of profiles, at $120,000+144$, $164,181,193,204,214$ and $222 \mathrm{~km}$, each of which roughly corresponds to a minimum in optical depth in the lower set of profiles. The phase $\phi$ of each profile (cf. Eq. 18) is labeled at lower right. 


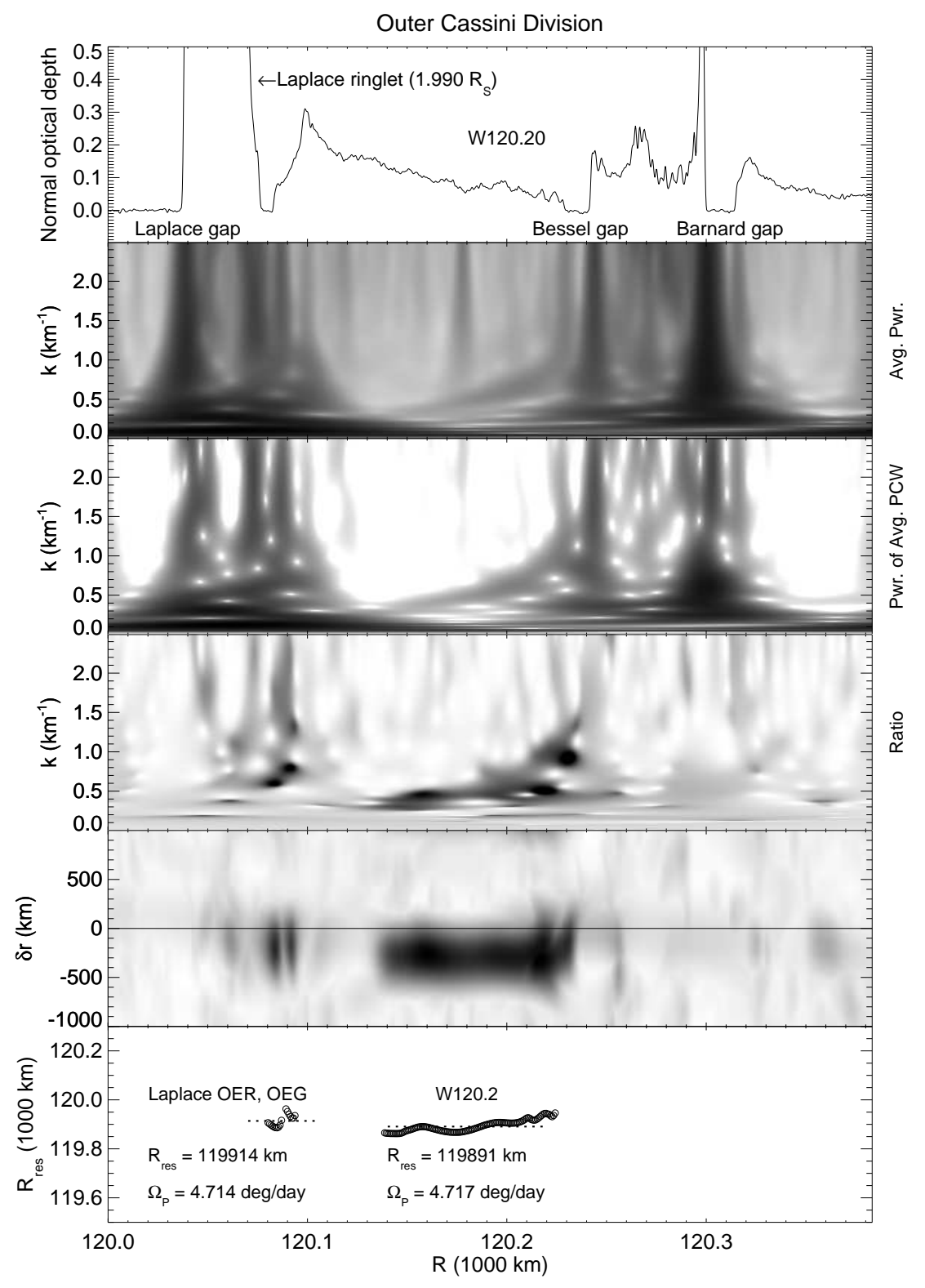

Figure 17. A survey of the outer Cassini Division using the phase-corrected wavelet algorithm, assuming density wave perturbations with $m=1$. The format is the same as that of Fig. 6. See the text in Section 4 for a detailed description of each panel. The signature of wave W120.2 is clearly seen in the third and fourth panels between radii of 120,140 and $120,230 \mathrm{~km}$. The bottom panel shows the resonant radius that corresponds to the best-fitting local pattern speed, as a function of radius.

radius $\sim 300 \pm 40 \mathrm{~km}$ interior to the wave itself, as shown in the fifth and sixth panels in Fig. 17. 


\subsection{W120.20 as a standing wave}

What is the nature of the prominent wavelike structure around 120,200 km? Figures 5 and 16 reveal a set of peaks in optical depth, alternating in true anomaly with troughs at the same radii, while Fig. 17 shows that this alternating pattern rotates at an average angular rate of $4.717^{\circ} \mathrm{d}^{-1}$, which matches the predicted apsidal precession rate for eccentric orbits at a radius of $\sim 119,890 \mathrm{~km}$.

Initially, we tried fitting the radii of the most prominent individual wave peaks in the RSS occultation data to simple models of precessing ellipses. We used the same algorithm employed by Nicholson et al. (2014a,b) and French et al. (2016a) to scan over a range of possible pattern speeds, in this case expected to be near the local value of $\dot{\varpi}_{\text {sec }} \simeq 4.7^{\circ} \mathrm{d}^{-1}$. An example of such a fit is shown in Fig. 18, which shows the RMS fit residuals vs the assumed pattern speed. In this case, the best-fitting ellipse has an amplitude $a e=2.2 \mathrm{~km}$ and a pattern speed of $4.705^{\circ} \mathrm{d}^{-1}$. The semimajor axis is $120,206 \mathrm{~km}$. Scans for nearby peaks yielded similar fits, with ae ranging from 1.6 to $2.5 \mathrm{~km}$ and $\Omega_{p}$ ranging from 4.70 to $4.74^{\circ} \mathrm{d}^{-1}$ (the latter are consistent with the results of the completely independent wavelet analysis shown in Fig. 17, albeit with somewhat larger scatter that we attribute to using a relatively small number of individual measurements of RSS features, rather than the full set of VIMS and RSS observations). Pericenters at epoch were within $20^{\circ}$ of that in Fig. 18. These fits convinced us that there is indeed a well-defined pattern speed for the structure, and that its basic nature is that of a set of regularly phased precessing elliptical orbits, but that it is neither a trailing nor a leading spiral pattern.

The true nature of this feature is best illustrated in Fig. 19 where we use a polar projection to show the location of individual wave crests and troughs in a frame rotating at the average observed pattern speed of $4.72^{\circ} \mathrm{d}^{-1}$. Filled circles indicate optical depth maxima, while crosses indicate the intervening minima. In this plot we show not only measurements from the RSS occultation data but also from VIMS stellar occultation profiles. What emerges is a picture of a standing wave, with an azimuthal wavenumber $m=1$ and an angular rotation rate $\Omega_{p} \simeq 4.72^{\circ} \mathrm{d}^{-1}$, but with a fixed circular pattern of radial nodes. A crest on one side of the pattern is at the same radius as a trough $180^{\circ}$ away, and vice versa. Unlike the case of a propagating wave, individual crests fall at a series of nearly constant radii, independent of longitude, rather than forming a spiral pattern. (In retrospect, our attempts to fit eccentric orbits to individual wave crests were instead fits to measurements of adjacent features in the square-wave pattern followed by each wave crest; it was this square-wave pattern in the observed radii of the crests that first alerted us to the possibility that this was a standing wave.) The existence of standing waves in Saturn's rings was first proposed by Borderies et al. (1985) for the dense B ring, with observational support from Cassini ISS images (Spitale and Porco 2010), but to our knowledge this is the 


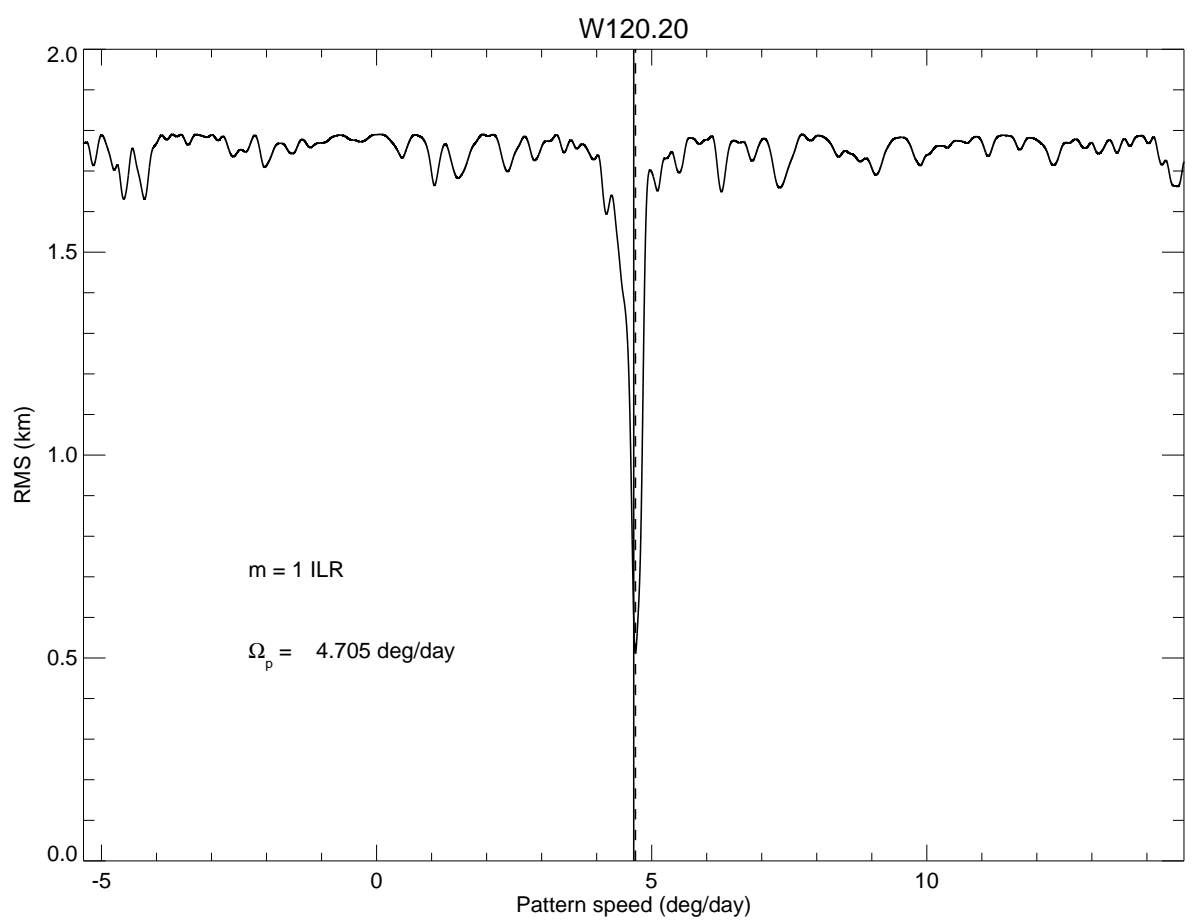

Figure 18. RMS radius residuals for an elliptical (i.e., $m=1$ ) fit to the single wave crest in W120.20 with a mean radius of $120,206 \mathrm{~km}$, assuming an $m=1$ pattern and plotted as function of the assumed pattern speed $\Omega_{p}$. For this fit we used only the RSS profiles shown in Fig. 5. The best-fitting ellipse has an amplitude $a e=2.2 \mathrm{~km}$ and a pattern speed of $4.705^{\circ} \mathrm{d}^{-1}$.

first direct observation of the detailed wave properties of a standing wave, and in a region of much lower optical depth than the $\mathrm{B}$ ring.

In the inertial frame, the pattern is also fixed in radius, but rotates at $4.72^{\circ} \mathrm{d}^{-1}$. At a given inertial longitude, the peaks therefore oscillate in sign with the same (angular) frequency, or a period of $2 \pi / \Omega_{p} \simeq 76.3 \mathrm{~d}$. It is thus a wave that propagates slowly in longitude but is stationary in radius, except for periodic alternations in the locations of individual peaks and troughs by $\pm \lambda_{r} / 2$, where $\lambda_{r}$ is the local radial wavelength of the wave.

Although edge modes and global modes of narrow ringlets are both standing waves of limited radial extent, to our knowledge no standing wave with such prominent periodic structure has previously been seen directly in a planetary ring. In some ways, however, it is not fundamentally different from the familiar propagating density waves. In fact, it can be visualized as a linear combination of two $m=1$ density waves: one a trailing spiral and the other a leading spiral, with the same pattern speed $\Omega_{p}$ and resonance location and with equal amplitudes. ${ }^{6}$ Both waves satisfy Eqns. (2), (3) and (4), but with opposite signs of the radial wavenumber $k(r)$. We can see this

${ }^{6}$ In fact, some of the numerical simulations by Hahn (2008) of $m=1$ density waves driven by a nearby moonlet developed into such standing waves, when the outbound trailing wave reflected at the outer boundary of the ring to form an inbound leading wave. 


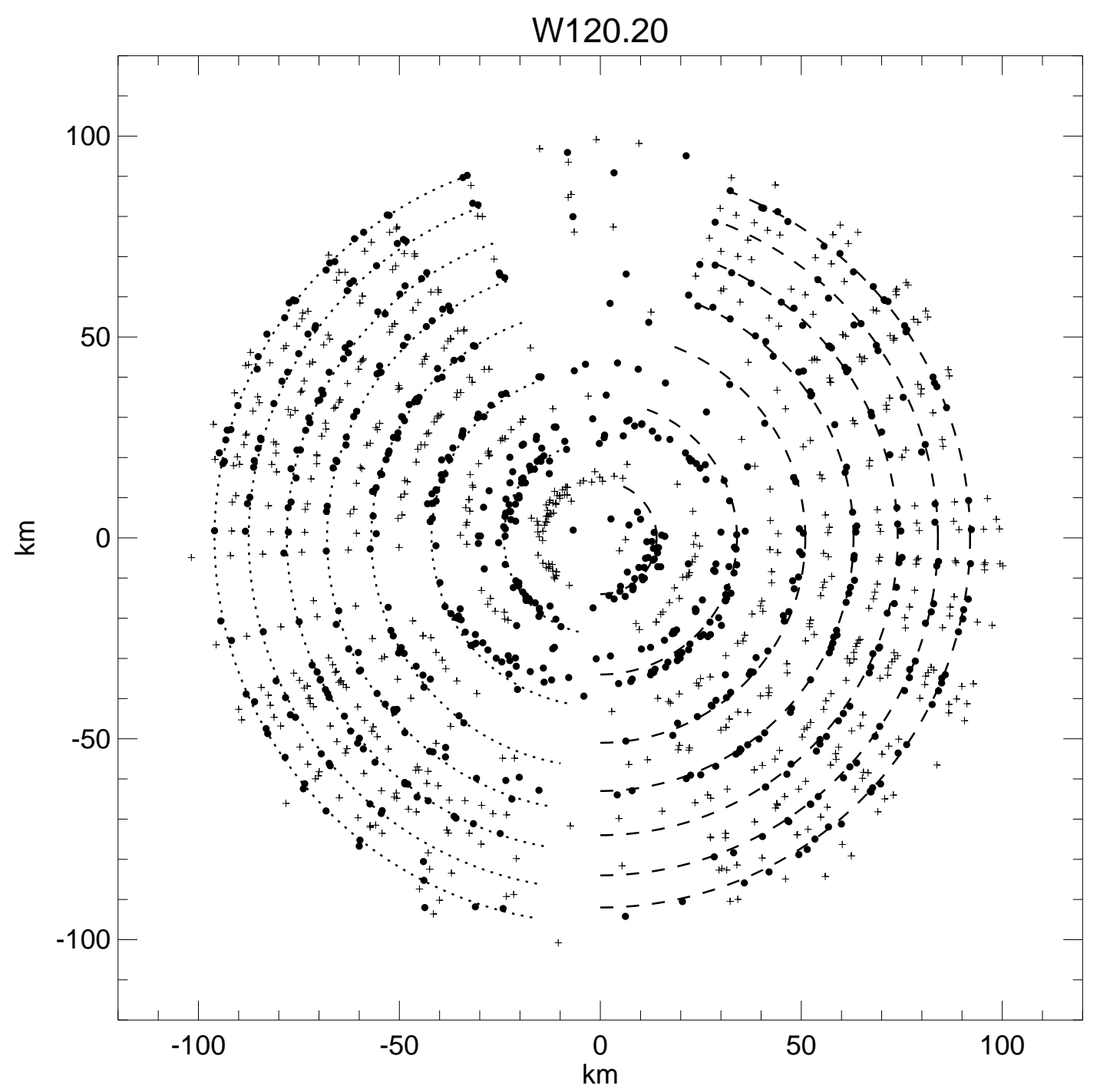

Figure 19. A polar projection $\left(r, \phi_{\lambda, t}\right)$ of the measured locations of wave crests and troughs for the wave W120.20 in the outer Cassini Division, as seen in a frame rotating at a uniform rate of $\Omega_{p}=4.72^{\circ} \mathrm{d}^{-1}$, with the origin at a radius of $120,130 \mathrm{~km}$ and the positive $\mathrm{x}$ axis corresponding to $\phi_{\lambda, t}=0$ (the phase at which the wave maxima reach their highest amplitude). In this coordinate system, the azimuthal nodes in the pattern of maxima and minima are oriented vertically. Filled circles indicate optical depth maxima, while crosses indicate the intervening minima. The dashed circular arcs are drawn at the same set of radii shown in Fig. 16, from 120,144 to $120,222 \mathrm{~km}$, and match the optical depth maxima on the right side of the diagram. Dotted circular arcs indicate radii midway between the dashed circular arcs, and match the optical depth maxima on the left side of the diagram.

by writing expressions, based on Eq. (1), for the variable parts of such trailing and leading waves, respectively, as

$$
\Delta \sigma_{T}(r, \lambda, t)=\sigma_{0} A(r) \mathcal{R}\left(e^{i\left(\phi_{\lambda, t}+\int|k(r)| d r\right)}\right)
$$




$$
\Delta \sigma_{L}(r, \lambda, t)=\sigma_{0} A(r) \mathcal{R}\left(e^{i\left(\phi_{\lambda, t}-\int|k(r)| d r\right)}\right)
$$

and then forming their sum

$$
\Delta \sigma_{S}(r, \lambda, t)=2 \sigma_{0} A(r) \cos \left(\int|k(r)| d r\right) \mathcal{R}\left(e^{i \phi_{\lambda, t}}\right) .
$$

Taking the real part of this expression, we have

$$
\Delta \sigma_{S}(r, \lambda, t)=2 \sigma_{0} A(r) \cos \left(\int|k(r)| d r\right) \cos \left[\lambda-\lambda_{0}-\Omega_{p}\left(t-t_{0}\right)\right],
$$

where we have set $m=1$ in the phase factor for our particular wave. This expression describes a standing wave with radial wavelength $2 \pi / k(r)$, modulated in longitude and time by the rotating factor $\cos \left[\lambda-\lambda_{0}-\Omega_{p}\left(t-t_{0}\right)\right]=\cos \phi_{\lambda, t}$ (after Eq. (2)).

We illustrate this graphically with a simple analytical model of W120.20 in Fig. 20, which is a single frame from a movie of W120.20 (see Supplementary Material). The left panel shows a trailing spiral, the middle panel shows the corresponding leading spiral, and their coaddition results in the standing wave shown in the final panel. Viewed in an inertial frame, the standing wave pattern rotates at a fixed pattern speed $\Omega_{p}$.

\subsubsection{ISS images of W120.20}

Given the novelty of such a feature, we have also looked for supporting evidence in ISS images. Figure 21 shows reprojected images of the W120.20 wave extracted from a pair of ISS/NAC images obtained toward the end of the Cassini mission in 2017 (see the bottom of Table 1 for image numbers and geometric parameters). The selected images sample the standing wave at nearly opposite phases $\left(\phi_{\lambda, t}=129.2^{\circ}\right.$ for image N1560311811 and $\phi_{\lambda, t}=333.0^{\circ}$ for image N1870073325, which differ by $156.2^{\circ}$ ), although neither image is near $\phi_{\lambda, t}=0$ or $180^{\circ}$. The corresponding radial brightness profiles are shown in Fig. 22, after subtraction of an average radial gradient in $I / F$. As well as clearly showing the standing wave, these very high SNR profiles also permit weaker oscillations to be traced inwards to a radius of $\sim 120,120 \mathrm{~km}$. This is possible because the $2 \mathrm{D}$ images can be averaged over longitude, greatly improving the SNR of the resulting radial profiles (Tiscareno and Harris 2018). The vertical dotted lines in Fig. 22 are drawn at the same radii as those in Figs. 16 and 19, confirming that the brightness maxima and minima in these individual images are located at nearly the same radii as the optical depth maxima and minima in the occultation profiles.

\subsubsection{W120.20 eccentricity gradient profiles from VIMS occultations}




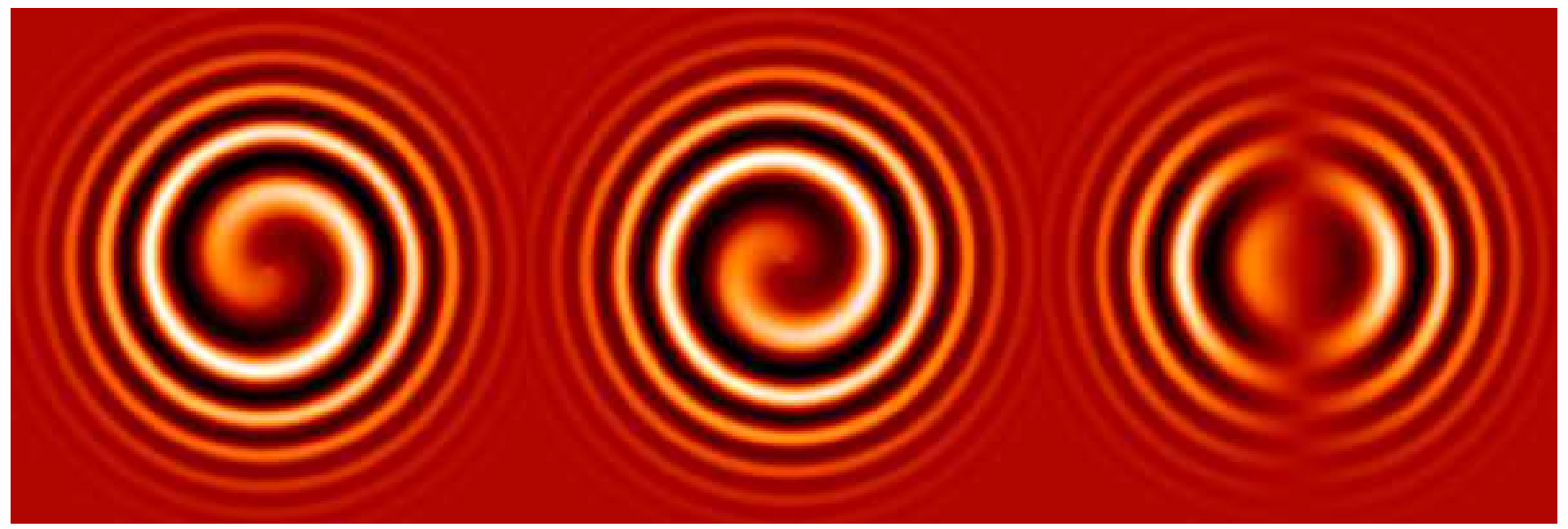

Figure 20. A single three-panel frame from a movie illustrating the decomposition of the standing wave W120.20 into leading and trailing spiral density waves. The left panel shows a trailing spiral wave with $m=1$, while the middle panel shows a leading $m=1$ spiral with the same amplitude and resonant radius. The right panel shows the standing wave that results from adding the leading and trailing waves. In inertial space, all three waves rotate counter-clockwise at the pattern speed $\Omega_{p}=4.72^{\circ} \mathrm{d}^{-1}$. The spiral waveforms are standard linear density waves, following the model of Shu (1984), with an artificial viscous damping term added. Compare the right panel with the measured peak and trough locations for wave W120.2 in Fig. 19. The complete movie is contained in the Supplementary Material.

The radial $I / F$ profiles in Figs. 21 and 22 qualitatively match the behavior of the RSS optical depth profiles in Fig. 16 and support the interpretation of W120.20 as a standing wave.

To investigate the wave structure more quantitatively, we now turn to a pair of high-SNR VIMS occultations of the same star (and hence at similar ring opening angle) that fortuitously sampled the wave crests and troughs at longitudes $\phi_{\lambda, t}$ near 0 and $180^{\circ}$. The upper panel of Fig. 23 shows the radial optical depth profiles for VIMS RLeo063 I $\left(\phi_{\lambda, t}=174.5^{\circ}\right)$ and RLeo077 I $\left(\phi_{\lambda, t}=1.3^{\circ}\right)$. 


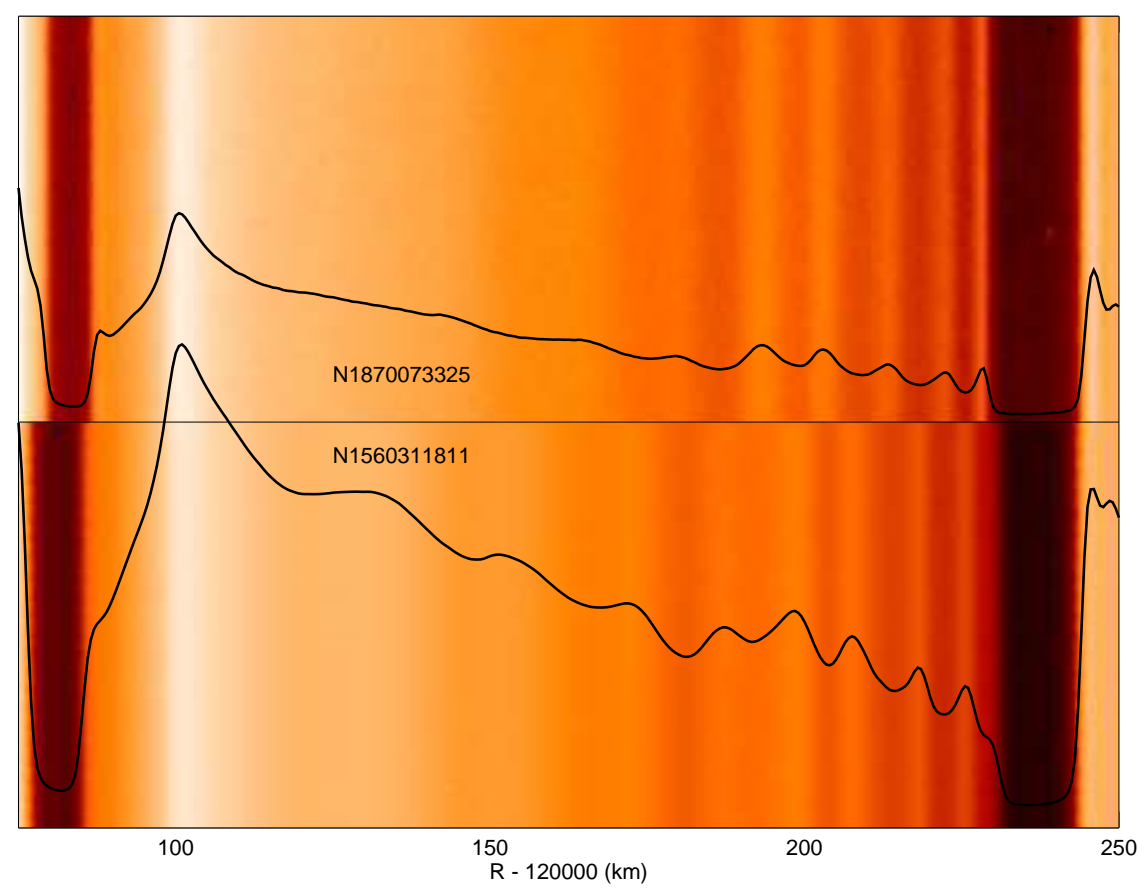

Figure 21. A pair of ISS images of the W120.20 standing wave, reprojected into radius and longitude. At the left edge of the images we see the slightly eccentric outer edges of the Laplace ringlet and gap, while at the right edge we see the eccentric inner edge of the narrow Bessel gap. Superimposed are radial profiles of brightness, averaged in longitude.

To characterize the radial and azimuthal behavior, we model the two-dimensional form of the standing wave as

$$
\tau\left(r, \phi_{\lambda, t}\right)=\frac{\bar{\tau}(r)}{1-q(r) \cos \phi_{\lambda, t}}
$$

where $\bar{\tau}(r)$ represents the azimuthally averaged radial variation of the mean normal optical depth and $q(r)$ incorporates the intrinsic radial structure of the standing wave itself, modulated sinusoidally in amplitude by the azimuthal dependence $\cos \phi_{\lambda, t}$. The form of Eq. (18) resembles the expression for the local surface density of a wake produced by a nearby satellite (see Eq. (2.1.10) of Showalter et al. (1986)), and is reminiscent of the streamline formalism of a non-linear density wave (cf. Eq. (24) of Rappaport et al. (2009)).

Although a detailed dynamical interpretation of a standing wave in a region of strongly varying background optical depth is beyond the scope of this work, the following physical interpretation seems plausible. For a standing wave, $q(r)$ is related to the eccentricity gradient of the wave, while the eccentricity itself is essentially the cosine of the underlying trailing wave radial phase (or equivalently of the reflected leading wave), multiplied by the underlying wave's own eccentricity. This would account for the rapid oscillations in $q$ from a kinematic point of view. Relatedly, the 


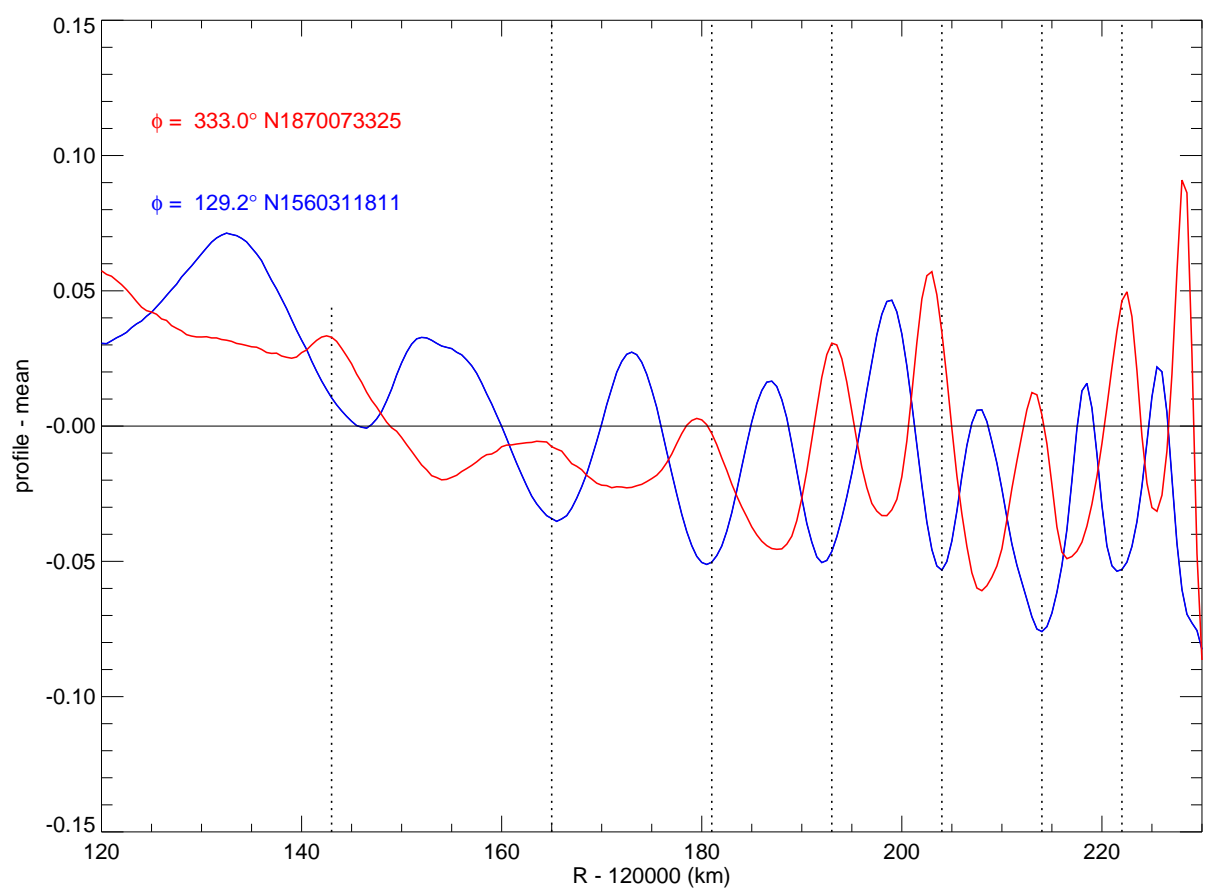

Figure 22. Radial brightness profiles extracted from the pair of ISS images in Fig. 21, after approximate removal of a background gradient. The $m=1$ phases of these two wave profiles differ by $156.2^{\circ}$, for an assumed pattern speed $\Omega_{p}=4.72^{\circ} \mathrm{d}^{-1}$. The vertical dotted lines are drawn at the set of radii derived from the RSS profiles shown in Figs. 16 and 19, and match the locations of the corresponding peaks for image N1870073325 within a $\mathrm{km}$ or so.

standing wave eccentricity changes sign at nodes, and successive nodes correspond to increases by $\pi$ of the underlying traveling wave radial phase. Under this view, the node structure should be closely related to the usual dispersion relation of density waves, which describes the radial dependence of their wavenumber (i.e., of the radial derivative of the wave phase). This shows up as a faster and faster oscillation of the eccentricity (and $q$ ) of the standing wave as one moves away from its resonance location.

As a rough approximation, we take $\bar{\tau}(r)$ to be the mean of the two VIMS profiles, resulting in the black curve included in the top panel of Fig. 23. ${ }^{7}$ We then solve Eq. (18) for $q(r)$ separately for each profile, resulting in the profiles shown in the bottom panel of Fig. 23. Notice the excellent agreement between the VIMS RLeo063 I and RLeo077 I $q(r)$ profiles, which we average and smooth to form $\bar{q}(r)$.

This analysis shows W120.20 to be a standing wave superimposed on a region of steeply decreasing opacity (and possibly surface mass density), with $\bar{\tau}(r)$ reaching a

${ }^{7}$ We favor this approach to the simple alternative of equating $\bar{\tau}(r)$ to $\tau(r)$ for a high-SNR occultation of a different star for $\phi_{\lambda, t}$ near $90^{\circ}$ or $270^{\circ}$, which would require introducing an empirical scaling factor to account for the differing opening angles of the occultations and the differing colors and angular diameters of the occulted stars. Our method results in some residual low-amplitude periodic structure in $\bar{\tau}(r)$ visible between 120,190 and $120,225 \mathrm{~km}$, but this does not significantly affect our conclusions. 

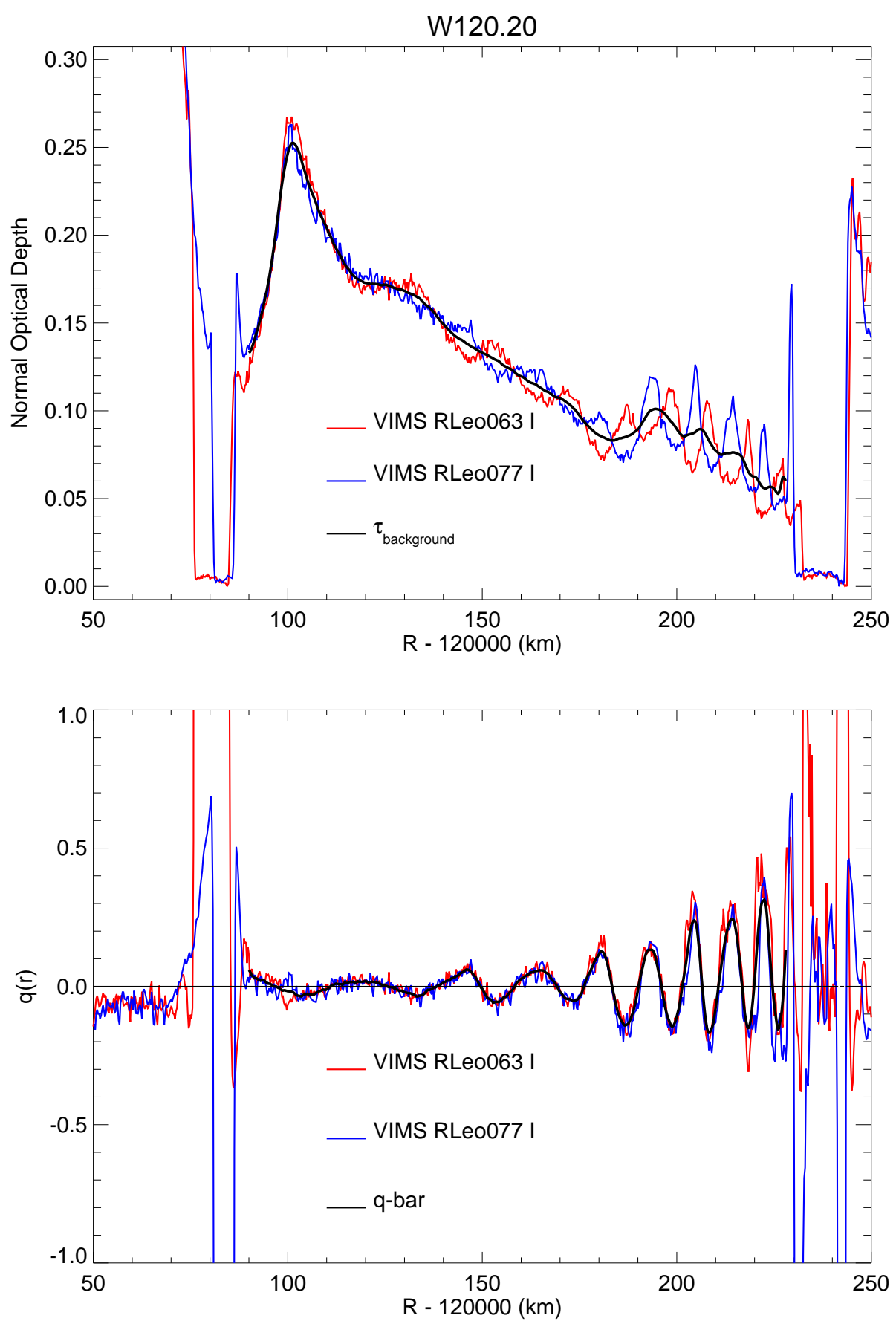

Figure 23. The background optical depth $\bar{\tau}(r)$ (upper panel) and intrinsic radial structure $q(r)$ (lower panel) of standing wave W120.20, from VIMS RLeo063 I and VIMS RLeo077 I radial occultation profiles obtained at nearly opposite phases of the wave. See text for details.

maximum of 0.25 at $120,100 \mathrm{~km}$ and diminishing by nearly a factor of five to 0.05 near $120,230 \mathrm{~km}$. We find that $q(r)$ increases gradually over this range, from $\sim 0.05$ to about 0.1 near 120,170 , and then more rapidly to over 0.5 as the wave reaches the inner edge of the Bessel gap near 120,231 km. Empirically, $q(r)$ scales roughly as 
$\bar{\tau}(r)^{-2}$, and the radial anti-correlation of $q(r)$ and $\bar{\tau}(r)$ (and, presumably, of surface density as well) is expected for a wave governed by the ring's self-gravity.

\subsection{A regional view of the outer Cassini Division}

Figure 24 summarizes all of the measured $m=1$ pattern speeds for the outer Cassini Division, drawing on the results of this paper as well as those of French et al. (2016a). As in Fig. 9, sharp edges of gaps and ringlets are indicated by filled circles, ${ }^{8}$ while the pattern speeds for the inner and outer parts of the W120.20 wave are shown as open circles, with error bars. The latter are based on Fig. 17. The diagonal line is the predicted apsidal precession rate $\dot{\varpi}_{\mathrm{sec}}$, based on Saturn's zonal gravity harmonics (Jacobson et al. 2008). As noted by French et al. (2016a), almost all pattern speeds are significantly higher than predicted in this region, for reasons that are currently unexplained. Particularly noticeable are the anomalously fast rates exhibited by the Laplace ringlet and outer edge of the Laplace gap as well as both edges of the Bessel gap.

For each of the $m=1$ waves or edges, a horizontal dotted line in Fig. 24 connects the measured pattern speed to the resonant radius, i.e., the radius where $\dot{\varpi}_{\mathrm{sec}}=\Omega_{p}$. These serve to emphasize an apparently close connection between the W120.20 wave and either the Laplace ringlet or the outer edge of the Laplace gap. All have pattern speeds of $4.72 \pm 0.01^{\circ} \mathrm{d}^{-1}$, despite a range in radius of more than $150 \mathrm{~km}$. We suggest that both the outer edge of the Laplace gap and the W120.20 wave represent $m=1$ modes that are forced by the nearby and relatively massive Laplace ringlet. More specifically, we propose that the outer edge of the Laplace gap first develops an eccentricity driven by the Laplace ringlet. This perturbation in turn drives an $m=1$ trailing density wave outwards from the gap edge. Under some circumstances, this wave would be expected to damp over a distance of several wavelengths, but in this case the wave reaches the Bessel gap, where it is reflected as a leading density wave with the same pattern speed. The leading wave then propagates inwards (Shu 1984), until it reaches the Laplace gap, where it reinforces the $m=1$ perturbation from the Laplace ringlet. Over time, the superposition of trailing and leading waves establishes a standing wave, effectively trapped in a resonant cavity between the Laplace and Bessel gaps, similar to the standing waves observed in numerical simulations by Hahn (2007, 2008).

Still unexplained, however, is the anomalously fast precession rate of the Laplace ringlet itself, as originally noted by French et al. (2016a), which seems more appropriate to the inner edge of the Laplace gap, $\sim 200 \mathrm{~km}$ closer to Saturn.

\footnotetext{
${ }^{8}$ Also included here is an $m=1$ fit to the sharp peak at 120,100 km, which appears to be eccentric. Fits to an extensive set of Cassini occultation observations yield $a e=0.23 \mathrm{~km}$ and a precession rate of $4.720^{\circ} \mathrm{d}^{-1}$, substantially faster than the predicted rate at this radius of $4.688^{\circ} \mathrm{d}^{-1}$ but closely matching the pattern speed of the nearby standing wave.
} 


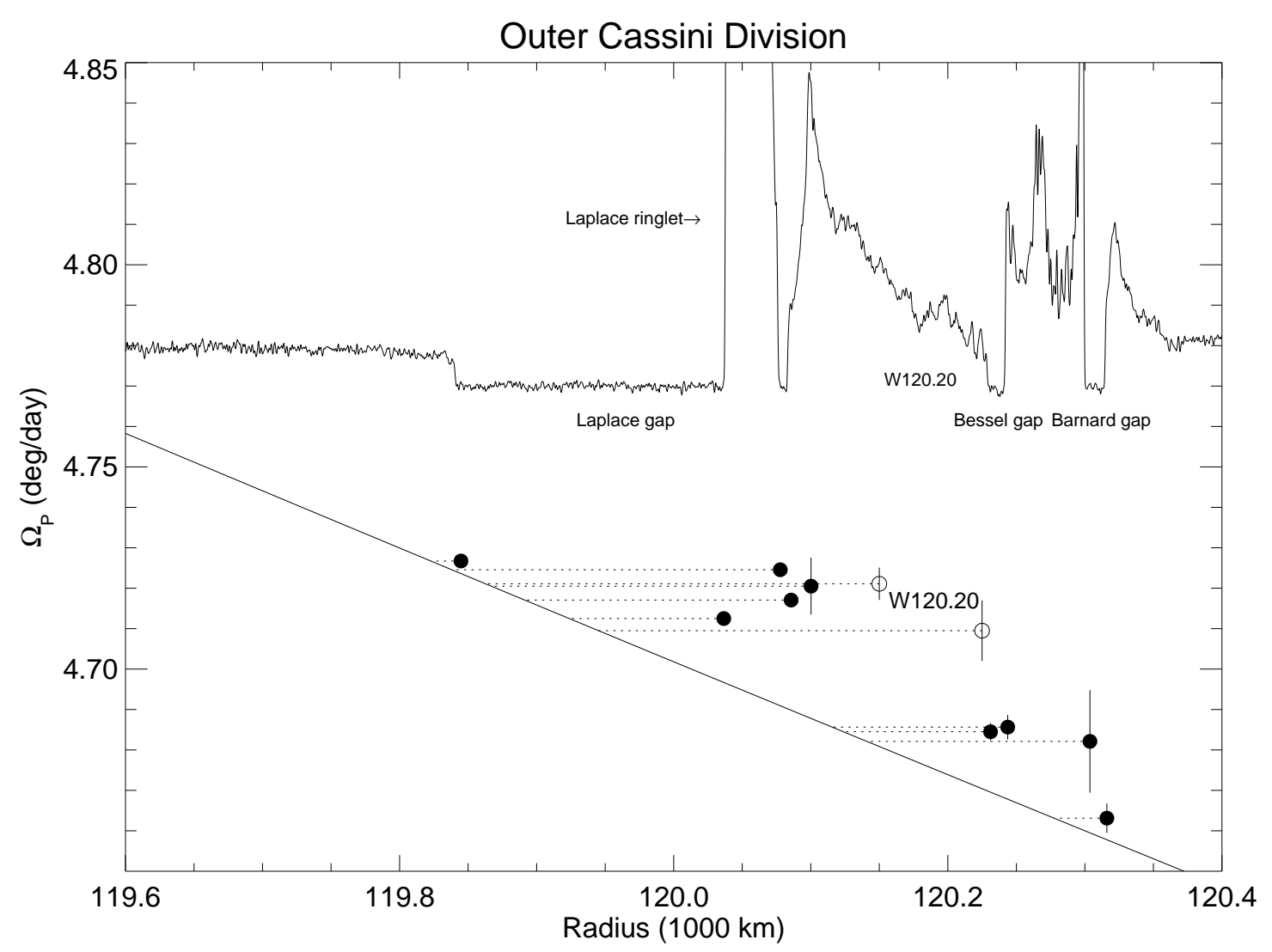

Figure 24. Regional $m=1$ pattern speeds in the outer Cassini Division. Filled circles denote gap and ringlet edges from French et al. (2016a) while open circles show the inner and outer portions of the W120.20 wave discussed in this paper. The diagonal line shows the apsidal precession rate $\dot{\varpi}_{\text {sec }}$ calculated from Saturn's zonal gravity harmonics. Horizontal dotted lines connect the measured pattern speeds for the gap or ringlet edges and waves to their corresponding resonant radii.

In the context of the above scenario, we recall that there is some evidence for a leading $m=1$ density wave in the outer part of Band 1, as well as the dominant trailing wave (see discussion of Fig. 8). This could be due to a similar situation whereby some of the outward-propagating energy in the trailing wave reaches the Herschel gap, where it is reflected as a leading spiral. But here the spacing between the gaps is larger (over $250 \mathrm{~km}$ vs $150 \mathrm{~km}$ ), which perhaps prevents the establishment of a true standing wave. In that case, the ultimate source of the waves appears to be forcing by the nearby Strange (R6) ringlet, as suggested in the discussion of Fig. 9 in Section 4.3 .

\section{DISCUSSION AND CONCLUSIONS}

\subsection{Summary of key results}

Using a large number of Cassini stellar and radio occultation observations, we have investigated several unusual density waves in Saturn's Cassini Division. In the inner 
Cassini Division, we identify outward-propagating density waves with wavenumber $m=1$ located in Band 1, at a radius of $\sim 118,050 \mathrm{~km}$ (designated W118.05) and in Band 2 at $\sim 118,400 \mathrm{~km}$ (W118.40) and $\sim 118,530 \mathrm{~km}$ (W118.53). Our model for the W118.05 wave is consistent with that proposed by Flynn and Cuzzi (1989) based on a much more limited set of Voyager observations.

We suggest that these waves may be driven by secular perturbations from nearby eccentric ringlets located interior to the waves: the Strange (or R6) ringlet in the case of W118.05 and the Herschel ringlet in the case of both W118.40 and W118.53. This scenario is closely akin to the model of secular perturbations by a nearby satellite on an eccentric orbit by Hahn (2008).

From Cassini ISS images taken near equinox at very low incidence angles we also find evidence for vertical structure in these waves, which we tentatively attribute to vertical splashing of ring material in the densest parts of the waves. Similar structures were produced in numerical simulations of moonlet wakes by Lewis and Stewart (2000), and Borderies et al. (1985) proposed that splashing could result when ring particles are so densely packed that they behave like an incompressible particle fluid, although it has not been shown that splashing can occur without close-packing, under the lower optical depth environment of the Cassini Division. The alternative of an outward-propagating bending wave, as modeled by Hahn (2007), is found to be inconsistent with the wave phases measured in our occultation profiles.

In the outer Cassini Division, we identify a third wavelike structure near 120,200 $\mathrm{km}$ (designated W120.20), between the Laplace and Bessel gaps, as the first known example of a standing wave in a planetary ring system. It appears to be the superposition of an outward-propagating $m=1$ trailing density wave, probably driven by the

nearby eccentric Laplace ringlet, and its reflection in the form of an $m=1$ leading wave from the inner edge of the Bessel gap.

Unlike most density waves, the W120.20 wave increases in amplitude with distance from its source. We attribute this to a factor-of-five decrease in the background optical depth across the wave region, with the wave's inferred $q(r)$ scaling roughly as $\bar{\tau}^{-2}(r)$.

\subsection{Evidence for ringlet-driven waves}

\subsubsection{The Cassini Division waves}

For the convenience of the reader, we summarize here the main characteristics of each wave, and the evidence that links each one to a specific ringlet. 
W118.05 is the most complex of the inner Cassini Division waves, showing evidence of some vertical structure and hints that the $m=1$ trailing spiral is partially reflected at the inner edge of the Herschel gap and propagates back towards the inner edge of Band 1 as a leading spiral. The best-fitting pattern speed of W118.05 of $\Omega_{p}=5.006 \pm$ $0.005^{\circ} \mathrm{d}^{-1}$ most closely matches the apsidal precession rate of the Strange ringlet, which is both eccentric and inclined, and thus potentially capable of producing the observed $m=1$ density wave structure and contributing to the less coherent vertical structure evident in the wave. However, the nearby more massive and eccentric Huygens ringlet has a comparable pattern speed, and would perhaps be expected to be the dominant driver of W118.05. This question is taken up in Section 7.4 below.

The two waves in Band 2 (W118.40 and W118.53) seem to form a single, coherent wave-train and their average pattern speed of $\Omega_{p}=4.958 \pm 0.005^{\circ} \mathrm{d}^{-1}$ best matches the apsidal precession rate of the nearby Herschel ringlet. However, the edges of the latter have quite small eccentricities (their average amplitude is $\overline{a e} \simeq 1.6 \mathrm{~km}$ ) and the models fitted by French et al. (2016a) suggest that they may precess independently, rather than remaining locked together like most eccentric ringlets (Nicholson et al. 2018). Moreover, the nearby inner edge of the Herschel gap has a similar precession rate and a much larger radial amplitude of $a e=8.27 \mathrm{~km}$. As with W118.05, these two waves exhibit signs of vertical structure, perhaps associated with the inclination of the Herschel ringlet itself (its edges have vertical amplitudes of $a \sin i=1.49-2.12 \mathrm{~km}$ ) or simply due to splashing.

W120.20 has an average pattern speed of $\Omega_{p}=4.717 \pm 0.005^{\circ} \mathrm{d}^{-1}$ that closely matches the average apsidal precession rate of the Laplace ringlet $\left(4.719^{\circ} \mathrm{d}^{-1}\right)$ and that of the outer edge of the Laplace gap $\left(4.717^{\circ} \mathrm{d}^{-1}\right)$. The mean amplitude of the former is $\overline{a e} \simeq 2.0 \mathrm{~km}$, while that of the latter is $a e=1.3 \mathrm{~km}$. As is the case for the Herschel ringlet, the inner and outer edges of the Laplace ringlet appear to precess independently. The inner edge of the Laplace gap is somewhat more eccentric, with $a e=3.3 \mathrm{~km}$, but it is much more distant and precesses considerably faster at $4.727^{\circ} \mathrm{d}^{-1}$. Except for the inner edge of the Laplace gap, all these features have pattern speeds that are much faster than expected for their radii, suggesting some sort of external influence in this region of the Cassini Division. This question will also be taken up in Section 7.4 below.

\subsubsection{Theoretical considerations}

All of the waves investigated in this study have anomalously high pattern speeds for their locations in the rings - that more closely match those of isolated eccentric ringlets that lie interior to the waves. This strongly suggests a dynamical association between the ringlets and the waves, though it does not prove a direct causal relationship. (Both the ringlet's precession rate and the wave's pattern speed might, 
for example, be driven by a common external agent.) Nevertheless, the properties of the Cassini Division spiral waves closely resemble those of a distinct class of $m=1$ spiral waves described by Hahn $(2007,2008)$. These studies considered the secular perturbations $^{9}$ that a gap-embedded satellite exerts on nearby ring material. But we note that the secular perturbations exerted by an orbiting point mass are identical to those exerted by a narrow ringlet with the same eccentricity and inclination, so the theory and numerical model developed in these papers can equally well be applied to the waves in the Cassini Division that appear to be driven by nearby eccentric ringlets.

Hahn (2008) showed that when the perturbing ringlet or satellite is eccentric, its secular perturbations raise the eccentricities of nearby ring material orbiting at both gap edges. Secular perturbations exerted by the now eccentric ring material then raise the eccentricities of adjacent ring particles, with that disturbance ultimately propagating radially outwards as a trailing $m=1$ density wave whose spiral pattern co-rotates with the apsidal line of the perturbing ringlet or satellite. In most cases, of course, the latter precesses due to the planetary oblateness. (Due to the dispersion relation obeyed by density waves, however, there is no equivalent inward-propagating wave interior to the gap.) The situation is analogous to a more typical density wave driven outward in the rings by an external satellite at an ILR, but here the pattern speed of the wave is much lower and the perturber is located at the Lindblad resonance, where Eq. (3) is satisfied for $m=1$. The dispersion relation is also modified by the gravity of the nearby satellite or ringlet. These secularly-driven spiral density waves resemble the observed waves in Bands 1 and 2, having a trailing $m=1$ wave pattern that propagates radially outwards while co-rotating with a precessing eccentric ringlet (either the Strange or Herschel ringlet, in our case) that is suspected of launching the observed wave.

Likewise, if the ringlet or satellite is inclined, its secular perturbations will drive inclinations on the adjacent gap edges, which will in turn launch a leading $m=1$ spiral bending wave that also propagates radially outwards and co-rotates with the regressing nodal line of the ringlet or satellite (Hahn 2007). (Again, there is no equivalent inward-propagating wave interior to the gap, because $m=1$ bending waves also propagate outwards, as noted in Section 5.2.) Superficially, such waves also resemble the waves seen in Bands 1 and 2, except that (1) there is no evidence of systematic leading or trailing wave behavior for W118.05 when plotted as a function of ascending node (see Fig. 12), and (2) our phase-corrected wavelet analysis failed to find any strong signal consistent with an $m=1$ bending wave with $\Omega_{p} \simeq \dot{\Omega}_{\text {sec }}$ (see Fig. 13).

\footnotetext{
${ }^{9}$ That is, those perturbations which are independent of the orbital phase of the satellite, but depend only on its apse and/or node.
} 
Hahn (2008) also considered waves launched by the small satellite Pan (which inhabits the Encke gap in the outer A ring), and argued that the hypothetical density waves launched by Pan would be unlikely to suffer significant viscous damping before reaching the Keeler gap $\sim 3000 \mathrm{~km}$ away. This is due to the very long wavelengths in the A ring, which are likely to be of order $100 \mathrm{~km}$ for $m=1$. A numerical simulation of Pan's hypothetical waves also showed that the waves are unable to jump the Keeler gap, despite its being only $\sim 40 \mathrm{~km}$ wide, but instead reflect at the gap's inner edge and propagate back inwards where they are superimposed upon the outbound waves. This particular simulation is rather reminiscent of the standing wave pattern observed in wave W120.20, though in the Cassini Division the radial wavelengths are only of order $10 \mathrm{~km}$. This suggests that a detailed comparison of model results to our observations, using a realistic background optical depth profile, could yield estimates of the surface mass density in the outer Cassini Division, as well as the presently-unknown mass of the Laplace ringlet.

The numerical code used to simulate these secularly-driven waves is available at https://github.com/joehahn/secular-waves, and that code is well-suited for testing the suggestion that the observed Cassini Division waves are in fact excited by secular perturbations from nearby ringlets.

\subsubsection{Searches for other ringlet-driven density waves}

More generally, if eccentric or inclined ringlets can produce $m=1$ density waves in exterior ring material, one can ask whether there are other comparable locations in Saturn's rings where one might search for evidence of similar waves. One such case is the Titan (or Colombo) ringlet, a prominent narrow feature in the outer third of the $170 \mathrm{~km}$-wide Colombo gap in the inner $\mathrm{C}$ ring, at a mean radius of $\sim 77,880 \mathrm{~km}$. It is on average $\sim 23 \mathrm{~km}$ wide, has a significant eccentricity, and is closely associated with the Titan 1: 0 resonance. As seen in Fig. 2 of Nicholson et al. (2014b), there is a $\sim 15 \mathrm{~km}$ wide ridge of material just outside of the gap edge that exhibits shortwavelength structure of unknown origin, but there is no clear indication of additional wave behavior in the nearby region outward of $77,930 \mathrm{~km}$, which has a relatively low normal optical depth $\tau \sim 0.08$, well below the optical depths of the Cassini Division wave regions.

Moving outward in the $\mathrm{C}$ ring, the next candidate is the Maxwell ringlet, located within the widest $(\sim 260 \mathrm{~km})$ gap in the $\mathrm{C}$ ring at a mean radius of $\sim 87,510 \mathrm{~km}$ (Nicholson et al. 2014b). Outward of the Maxwell gap lies a broad and nearly featureless low-optical depth region $(\tau \sim 0.05)$ extending to about 88,300 km, again showing no evident wave structure. (The weak Atlas 2:1 density wave falls just outside the Maxwell gap at $87,647 \mathrm{~km}$, but its wavelength is much shorter than an $m=1$ wave would be.) 
Next, we reach the Bond ringlet, with a mean radius of $\sim 88,710 \mathrm{~km}$ and a width of $\sim 17 \mathrm{~km}$, tightly nestled within the $37 \mathrm{~km}$-wide Bond gap (Nicholson et al. 2014b). The ring material external to the outer edge of the gap has a mean optical depth of about 0.05. Rappaport/Borderies (1998) investigated the gravitational perturbations exerted by the ringlet on test particles orbiting at the gap edges to explore possible mechanisms of gap confinement. There is a prominent wave between 88,725 and $88,760 \mathrm{~km}$ (feature 35 on a list of C ring waves cataloged by Baillié et al. (2011)) and subsequently identified by Hedman and Nicholson (2014) as an $m=1$ ILR, possibly driven by the Bond ringlet. This seems to be a candidate for the same driving mechanism as the Cassini Division waves. However, while the edges of the Bond ringlet exhibit a dizzying array of normal modes (see Table 3, Nicholson et al. (2014b)), the ringlet has no measurable inclination or eccentricity.

The final $\mathrm{C}$ ring candidate is the Dawes ringlet, a broad plateau-like feature 62 $\mathrm{km}$ wide with modest eccentricity $(a e \sim 1.35 \mathrm{~km}$ ) bounded at the outer edge at $\sim 90,200 \mathrm{~km}$ by the inner edge of the narrow ( $\sim 20 \mathrm{~km}$ wide) Dawes gap (Nicholson et al. 2014b). As in the case of the Maxwell gap, the outer edge of the Dawes gap shows evidence of an eccentricity that may be sympathetically forced by the ringlet interior to it. Baillié et al. (2011) found wavelike structure within the ringlet (their feature 39) and in the low optical depth region $(\tau \sim 0.05) 80 \mathrm{~km}$ outside of the gap (their feature 40).

On balance, this survey of narrow ringlets in the $\mathrm{C}$ ring serves primarily to underscore the desirability of a more detailed and quantitative investigation of the dynamics of ringlet-driven waves in Saturn's rings, perhaps using the computational tools of Hahn (2007) and Hahn (2008), tuned to the particular circumstances of the C ring candidates and Cassini Division waves explored here.

\subsection{Standing waves and normal modes}

Waves such as W120.20 may exist only in unusual circumstances where there are (1) two nearby gaps to provide a suitable cavity, and (2) a nearby eccentric ringlet or satellite to excite the $m=1$ perturbation in the first place, The W120.20 resonant cavity lies between the outer edge of the Laplace gap (at $\sim 120,086 \mathrm{~km}$ ) and the inner edge of the Bessel gap (at $\sim 120,231 \mathrm{~km}$ ), and the standing wave extends over 8 or 9 wavelengths. The forcing is presumed to be due to the nearby optically-thick and eccentric Laplace ringlet, located in the outer part of the Laplace gap.

Although such long-wavelength standing waves appear to be rare in Saturn's rings, they were predicted to exist in dense regions based on models of granular flow (Borderies et al. 1985), and we note that the overall scenario has similarities to that proposed to explain the existence of the numerous normal modes observed on the 
edges of gaps and ringlets throughout Saturn's ring system (Spitale and Porco 2010; Nicholson et al. 2014a,b; French et al. 2016a). In the case of edge modes, which are seen for many different values of $m$, the resonant cavity is bounded on one side by the ringlet or gap edge and on the other by a virtual Lindblad resonance, whose exact location is determined by the pattern speed of the mode. Typical inferred cavity widths are only a few radial wavelengths, or $\sim 5 \mathrm{~km}$ in the $\mathrm{C}$ ring (Nicholson et al. 2014b) and Cassini Division (French et al. 2016a), but up to several tens of $\mathrm{km}$ at the outer edge of the B ring (Nicholson et al. 2014a). (For $m=1$ modes, however, the radial wavelengths and thus cavity widths can be much larger - see discussion after Eq. (4).)

Some edge modes are driven by resonances with external satellites, notably the $m=2$ mode(s) at the outer edge of the B ring (Spitale and Porco 2010; Nicholson et al. 2014a) and the $m=7$ mode at the outer edge of the A ring (Spitale and Porco 2009; El Moutamid et al. 2016), but the origins of most are unknown.

Another instance of standing waves in rings may be the $m=0$ and $m=2$ normal modes observed in the narrow $\gamma$ and $\delta$ rings of Uranus (French et al. 1991; Nicholson et al. 2018) . These modes are thought to be self-excited, with probably at most one-half of a radial wavelength fitting between the inner and outer ring edges, so that the whole ring oscillates coherently in radius (Longaretti and Rappaport 1995).

\subsection{Anomalous precession rates}

The numerous instances of anomalous precession rates of ringlets and gap edges found in the Cassini Division and seen in Figs. 9 and 24 raise some questions in relation to the picture of ringlet-driven waves proposed in this paper. These precession anomalies are most clearly visible in the Huygens gap outer edge (see Fig. 9) and the Laplace ringlet region (see Fig. 24). Their magnitudes are comparable to the level of perturbation needed to enforce the pattern speeds of the various waves we have identified. Consequently, one may expect that whatever creates these anomalies may also perturb the structure of these waves. One may also ask if the sources of these anomalies might not generate the waves in the first place. We briefly discuss this last question at the end of this section.

There are at least two possible generic sources of precession anomalies in the Cassini Division: local structures such as nearby ringlets and/or edge modes, or more distant ones, the multiple B ring edge modes identified by Spitale and Porco (2010) and Nicholson et al. (2014a) being the most prominent option. We will start by examining local sources, leaving aside the Herschel ringlet and associated wave, as according to Fig. 9 none of the associated features seems to exhibit a significant precession rate anomaly. 
In the vicinity of the Huygens and Strange ringlets, the most obvious problem stems from the Huygens gap's outer edge: the fact that this edge has a different precession rate than the W118.05 wave's pattern speed implies the existence of an $m=1$ edge mode in addition to the $m=1$ wave. If the Strange ringlet is indeed the source of the wave, as suggested by the close match of the wave pattern speed with this ringlet's precession rate, an order of magnitude estimate based on an analysis by Borderies et al. (1983) of a similar system suggests that this ringlet may also significantly perturb the precession rate of this edge mode, leading to long-term variations in ae and in $\dot{\varpi}$. The problem then is that the Huygens ringlet should also perturb the Strange ringlet, the edge mode and the wave - and possibly generate a wave on its own - but no such perturbation is seen in the Strange ringlet's precession rate. It may turn out that the combination of the Huygens ringlet perturbation and of the edge mode and wave back-reactions on the Strange ringlet's precession rate just happen to cancel out in the time period of the Cassini observations, but this seems rather contrived. In any case, whatever is going on here, and in the absence of other sources of perturbation, these considerations do not seem to invalidate the idea that one or the other ringlet does generate the observed wave.

The Laplace ringlet/W120.20 region is quite puzzling as well. The Laplace ringlet is close enough to the Laplace gap's outer edge to generate the wave, and the ringlet's mean precession rate seems to match the average pattern speed of the wave. This ringlet may even generate the other precession anomalies seen in Fig. 24. But nothing in the outer Cassini Division region seems able to perturb the Laplace ringlet's precession rate itself at the observed level.

Conversely, the $\mathrm{B}$ ring edge (e.g., the $m=1$ edge mode) may be the primary cause of the precession anomalies in the outer Cassini Division. In this case, one would naively expect to observe a global trend in precession anomalies across the Cassini Division. However, Figs. 9 and 24 do not display any such trend, which requires further explanation for this possibility to be viable. One may invoke several processes on this front. First, this type of perturbation is time-dependent and its period depends on the distance to the B ring edge (Borderies et al. 1983), because precession locking does not occur in this case (unlike what happens if the same source drives a wave). For this reason, the perturbation may turn out to be weaker in the intermediate Cassini Division region where precession anomalies are not significant for the most part. Second, there are several other potential sources of $m=1$ perturbations at the B ring edge (such as those due to first order, non-secular coupling between $m$ and $m \pm 1$ edge modes, similar to those invoked by Hedman and Nicholson (2019) to account for the existence of $m=0$ waves), and their uncorrelated time-dependent behavior may be additive in some places and mutually canceling in others. Finally, local Cassini Division structures will perturb each others' precession rates, as discussed earlier. 
These effects may be somewhat coincidental, but jointly, they may disrupt to some extent any global trend in the precession anomalies.

One may also ask if the perturbations due to B ring edge modes could not provide an alternative source for the waves identified in this paper. For example, it is conceivable that the W120.20 wave might be excited in this way if some B ring edge feature has the appropriate pattern speed (e.g., one of the disturbances due to the first order coupling mentioned above), albeit in a time-dependent fashion. However, the associated perturbation would also be able to drive waves anywhere in the Cassini Division with the same pattern speed, whereas the waves identified so far all have different pattern speeds. (Conversely, ringlet perturbations are only significant for ring material in their immediate vicinity and, unavoidably, the waves they excite will have different pattern speeds.) In any case, the existence of perturbations on the B ring edge with the appropriate pattern speeds still needs to be ascertained.

In the latter context, we might recall the suggestion of Hedman et al. (2010) that the locations and precession rates of most of the eccentric gap and ringlet edges in the Cassini Division might be due to a series of three-body resonances involving the slow libration of the large $m=2$ perturbation at the outer edge of the $\mathrm{B}$ ring. This hypothesis remains unverified, but serves to reinforce the possible role played by the $\mathrm{B}$ ring edge throughout the Cassini Division.

This short discussion indicates that the most obvious sources of precession anomalies all raise self-consistency conundrums, at least superficially. However, we have not yet identified any compelling reason to question the view put forward in this paper, i.e., that eccentric ringlets or ringlet edge modes generate the identified $m=1$ density waves observed in nearby Cassini Division material.

\subsection{Concluding remarks}

The unusual density waves in the Cassini Division identified in this work reside in a complex dynamical environment. More detailed dynamical investigations, numerical simulations, and additional observational constraints on the detailed orbital properties of the nearby ringlets from Cassini occultation measurements late in the orbital tour are all likely to clarify our understanding of these striking features. The recent hydrodynamical simulations by Lehmann et al. (2019) of wave propagation through inhomogeneous ring structure represent a significant step in this direction.

\section{APPENDIX A}

We examine here the conditions for an inclined feature in the rings to fall within the shadow of a nearby gap edge, when the solar elevation angle $B_{\odot}$ is very low. We 
denote the radius of the inclined feature from the planet's center by $r_{i}$ and that of the nearby gap edge by $r_{e}$, which may be either smaller or larger than $r_{i}$. The inertial longitudes of the observation point and of the direction towards the sun are denoted by $\lambda$ and $\lambda_{\odot}$. By convention, the hour angle $H$ is measured from local midnight on the rings, so that we have $H=\lambda-\lambda_{\odot}+180^{\circ}$. The radial length of the shadow cast on a flat ring by an object of height $z$ may be written $\Delta r=-z / \tan B_{\text {eff }}$, where the effective elevation angle of the sun is given by Eq. (13):

$$
\tan B_{\text {eff }}=\frac{\tan B_{\odot}}{\cos \left(\lambda-\lambda_{\odot}\right)}=-\frac{\tan B_{\odot}}{\cos H}
$$

In terms of the usual Keplerian orbital elements $a, e, i, \varpi$ and $\Omega$, the local radius and height of the inclined feature are given approximately by

$$
r_{i} \simeq a[1-e \cos (\lambda-\varpi)]
$$

and

$$
\left.z_{i} \simeq r_{i} \sin i \sin (\lambda-\Omega)\right]
$$

In our situation, rather than the inclined feature casting its shadow upon the flat (i.e., uninclined) ring, we are interested in whether or not the inclined feature falls within the shadow of a nearby flat, semi-infinite ring. Reversing the above expression, we find that the height of the shadow cast by the ring edge at a radial distance $\Delta r=r_{i}-r_{e}$ from the edge is given by $z_{\text {shad }}=\Delta r \tan B_{\text {eff }}$. We then have four possible situations to consider, depending on the sign of $B_{\odot}$ and on whether we are observing the daytime $\left(90^{\circ}<H<270^{\circ}\right)$ or nightime $\left(270^{\circ}<H<90^{\circ}\right)$ side of the rings, i.e., whether $\cos H$ is negative or positive.

- $B_{\odot}>0$ and $\cos H<0$ : the shadow is cast inward and the inclined feature is in shadow if $z_{i}<z_{\text {shad }}$. (Note that in this case $B_{\text {eff }}>0$ and $\Delta r<0$, so that the RHS of this expression is negative.)

- $B_{\odot}>0$ and $\cos H>0$ : the shadow is cast outward and the inclined feature is in shadow if $z_{i}<z_{\text {shad }}$. (Note that in this case $B_{\text {eff }}<0$ and $\Delta r>0$, so that the RHS of the expression is again negative.)

- $B_{\odot}<0$ and $\cos H<0$ : the shadow is cast inward and the inclined feature is in shadow if $z_{i}>z_{\text {shad }}$. (Note that in this case $B_{\text {eff }}<0$ and $\Delta r<0$, so that the RHS of the expression is positive.)

- $B_{\odot}<0$ and $\cos H>0$ : the shadow is cast outward and the inclined feature is in shadow if $z_{i}>z_{\text {shad }}$. (Note that in this case $B_{\text {eff }}>0$ and $\Delta r>0$, so that the RHS of the expression is again positive.) 
Table 2. Huygens ringlet shadow geometry

\begin{tabular}{|c|c|c|c|c|c|c|c|}
\hline Image & Shadow direction & $B_{\text {eff }}(\operatorname{deg})$ & Ringlet edge & $\Delta r(\mathrm{~km})$ & $z_{i}(\mathrm{~km})$ & $z_{\text {shad }}(\mathrm{km})$ & Shadowed? \\
\hline \multirow[t]{2}{*}{ N1495327885 } & Outward & 52.51 & Inner & 55.36 & -0.62 & 72.17 & $\mathrm{~N}$ \\
\hline & & & Outer & 82.01 & -1.52 & 106.91 & $\mathrm{~N}$ \\
\hline \multirow[t]{2}{*}{ N1595342033 } & Outward & 6.88 & Inner & 47.11 & 0.12 & 5.69 & $\mathrm{~N}$ \\
\hline & & & Outer & 77.52 & -0.59 & 9.36 & $\mathrm{~N}$ \\
\hline \multirow[t]{2}{*}{ N1625956787 } & Inward & -0.48 & Inner & -49.43 & 0.26 & 0.41 & $\mathrm{~N}$ \\
\hline & & & Outer & -22.91 & 1.08 & 0.19 & $\mathrm{Y}$ \\
\hline \multirow[t]{2}{*}{ N1627303569 } & Outward & 0.29 & Inner & 39.72 & 1.44 & 0.20 & $\mathrm{Y}$ \\
\hline & & & Outer & 72.04 & 2.12 & 0.37 & $\mathrm{Y}$ \\
\hline \multirow[t]{2}{*}{ N1627307734 } & Outward & 0.35 & Inner & 37.83 & 1.33 & 0.23 & $\mathrm{Y}$ \\
\hline & & & Outer & 69.81 & 2.11 & 0.42 & $\mathrm{Y}$ \\
\hline
\end{tabular}

In each case we must have $\left|z_{i}\right|>\left|z_{\text {shad }}\right|=\left|\Delta r \tan B_{\text {eff }}\right|$ if the inclined feature is to be in shadow, but the sign of $z_{i}$ also needs to be right: negative if $B_{\odot}>0$ and positive if $B_{\odot}<0$.

Table 2 lists the values of $B_{\text {eff }}, \Delta r, z_{i}$ and $z_{\text {shad }}$ for both edges of the Herschel ringlet, as shadowed by the inner or outer edge of its gap - depending on the sign of cos $H$ — for each of the images in Fig. 14. We see that $\left|z_{i}\right| \ll\left|z_{\text {shad }}\right|$ for the first two images and that no shadowing is possible. For the third image, N1625956787, $B_{\odot}$ and $\cos H$ are both negative, and the ringlet can be shadowed by the outer gap edge only if $z_{i}>z_{\text {shad }}$. We find that $z_{i}$ is indeed positive, but only larger than $z_{\text {shad }}$ for the outer edge of the ringlet. This probably accounts for the relative faintness of the outer part of the ringlet in Fig. 15.

For the fourth and fifth images, N1627303569 and N1627307734, $B_{\odot}<0$ and $\cos H>0$, and the ringlet can be shadowed by the inner gap edge only if $z_{i}>z_{\text {shad }}$. Here we find again that $z_{i}$ is positive, near its maximum value for each edge, and substantially larger than $z_{\text {shad }}$ for both ringlet edges. We thus predict that the entire ringlet will be in shadow in both images.

In the case of narrow gaps with no ringlets, shadowing of one gap edge by the other is possible, as long as one of the edges is sufficiently inclined. For the case at hand, we suspect that the inner edges of the Russell and Jeffreys gaps are in shadow in images N1627303569 and N1627307734, while the outer edges are not. Furthermore, both gaps appear to have slightly inclined inner edges, but circular, zero-inclination outer edges (see French et al. (2016a), Table 1). (We have revisited these models, using an expanded data set, and find a small but marginally significant vertical amplitude for 
the Russell gap's inner edge of $a \sin i=0.076 \pm 0.012 \mathrm{~km}$, with a somewhat larger value of $a \sin i=0.169 \pm 0.013 \mathrm{~km}$ for the Jeffreys gap.)

For image N1625956787, the shadowing direction is inward, and the inner gap edge can be shadowed by the outer gap edge only if $z_{i}>z_{\text {shad }}$. Since $\Delta r \simeq-38 \mathrm{~km}$ for both gaps, we find that $z_{\text {shad }} \simeq 0.30 \mathrm{~km}$, which is at least a factor of two greater than the maximum possible value of $z_{i}$. No shadowing of the inner edge is possible, in agreement with Fig. 15.

For images N1627303569 and N1627307734, on the other hand, $B_{\odot}<0$ and $\cos H>$ 0 , and the shadow is cast outwards. There is thus no way for the inner edge to be in shadow, unless it effectively shadows itself. This can happen if the slope of the ring adjacent to the edge $S$ is greater than $B_{\text {eff }}$, or $\sim 0.35^{\circ}$. Although we have no real idea of the ring's vertical profile away from the gap edges, we can approximate this crudely as $S \simeq z_{i} / L$, where $L$ is the radial e-folding distance over which the inclination decays to zero. Both inner gap edges are displaced upwards at the observed longitudes, a necessary condition for the gaps to shadow themselves. Setting $z_{i}$ to the values calculated from orbit models for the gaps for the appropriate image geometry $(0.05$ and $0.10 \mathrm{~km}$ for the Russell and Jeffreys gaps, respectively), we find that $S>B_{\text {eff }}$ only if $L<10 \mathrm{~km}$ for the Russell gap and $L<20 \mathrm{~km}$ for the Jeffreys gap. These values are roughly of the same order as the first wavelength of $m=1$ density or bending waves in the Cassini Division (Flynn and Cuzzi 1989), lending some support to the possibility that both gaps shadow themselves in these two images.

Finally, we note that the relatively high spacecraft elevation angle for all of the images used here (at least $30^{\circ}$ for the near-equinox images) means that any apparent changes in gap or ringlet widths must be due to the unusual illumination conditions rather than to the viewing geometry.

\section{ACKNOWLEDGEMENTS}

We are grateful to Robert French and Joe Spitale for providing detailed examples of ISS image navigation that guided the development of our own algorithms for image steering, and to Mitch Gordon for assistance with the PDS Ring-Moon Systems Node's OPUS search service. We are grateful to two anonymous reviewers for their helpful suggestions, and for alerting us to the recent dynamical simulations of Lehmann et al. (2019). This work was supported in part by the Cassini project and by NASA CDAP grants NNX09SE66G and 80NSSC10K0890. P.Y.L. acknowledges support by the French National Program of Planetology (PNP). JMH's contributions were supported by NSF AST grant 1313013.

\section{DATA AVAILABILITY}


All of the occultation data from which our results were obtained are publicly available from NASA's Planetary Data System Ring-Moon Systems Node at https://pds-rings.seti.org/ringocc/. The full movie of Fig. 20 is included as Supplementary Material.

\section{REFERENCES}

Baillié, K., Colwell, J. E., Lissauer, J. J., Esposito, L. W., Sremčević, M. 2011.

Waves in Cassini UVIS stellar occultations. 2. The $\mathrm{C}$ ring. Icarus 216 , 292-308.

Borderies, N., Goldreich, P., Tremaine, S. 1983a. The variations in eccentricity and apse precession rate of a narrow ring perturbed by a close satellite. Icarus 53, 84-89.

Borderies, N., Goldreich, P., Tremaine, S. 1983b. The dynamics of elliptical rings. The Astronomical Journal 88, 1560-1568.

Borderies, N., Goldreich, P., Tremaine, S. 1985. A granular flow model for dense planetary rings. Icarus 63, 406-420.

Colwell, J. E., Nicholson, P. D., Tiscareno, M. S., Murray, C. D., French, R. G., Marouf, E. A. 2009. The structure of Saturn's rings. In "Saturn after Cassini-Huygens." M. Dougherty \& S. Krimigis, Eds., Springer. 375-412. Colwell, J. E., Cooney, J. H., Esposito, L. W., Sremčević, M. 2009. Density waves in Cassini UVIS stellar occultations. 1. The Cassini Division. Icarus 200, 574-580.

Cooke, M. L. 1991. Saturn's rings:

Photometric studies of the $\mathrm{C}$ ring and radial variation in the Keeler gap. Ph.D. Thesis.

Cuzzi, J. N., Lissauer, J. J., Esposito, L. W., Holberg, J. B., Marouf, E. A., Tyler, G. L., Boishchot, A. 1984. Saturn's rings - Properties and processes. IAU Colloq. 75: Planetary Rings 73-199.

Cuzzi, J. N., Scargle, J. D. 1985. Wavy edges suggest moonlet in Encke's gap. The Astrophysical Journal 292, 276-290.
El Moutamid, M., Nicholson, P. D., French, R. G., Tiscareno, M. S., Murray, C. D., Evans, M. W., French, C. M., Hedman, M. M., Burns, J. A. 2016. How Janus' orbital swap affects the edge of Saturn's A ring? Icarus 279, 125-140.

Esposito, L. W., Cuzzi, J. N., Holberg, J. B., Marouf, E. A., Tyler, G. L., Porco, C. C. 1984. Saturn's rings Structure, dynamics, and particle properties. Saturn 463-545.

Flynn, B. C., Cuzzi, J. N. 1989. Regular structure in the inner Cassini Division of Saturn's rings. Icarus 82, 180-199.

French, R. G., Marouf, E. A., Rappaport, N. J., McGhee, C. A. 2010. Occultation observations of Saturn's B ring and Cassini Division. The Astronomical Journal 139, 1649-1667.

French, R. G., Nicholson, P. D., Porco, C. C., Marouf, E. A. 1991. Dynamics and structure of the Uranian rings.. Uranus 327.

French, R. G., Nicholson, P. D., McGhee-French, C. A., Lonergan, K., Sepersky, T., Hedman, M. M., Marouf, E. A., Colwell, J. E. 2016a. Noncircular features in Saturn's rings III: The Cassini Division. Icarus 274, 131-162.

French, R. G., Nicholson, P. D., Hedman, M. M., Hahn, J. M., McGhee-French, C. A., Colwell, J. E., Marouf, E. A., Rappaport, N. J. 2016b. Deciphering the embedded wave in Saturn's Maxwell ringlet. Icarus 279, 62-77. 
French, R. G., McGhee-French, C. A., Lonergan, K., Sepersky, T., Jacobson, R. A., Nicholson, P. D., Hedman, M. M., Marouf, E. A., Colwell, J. E. 2017. Noncircular features in Saturn's rings IV: Absolute radius scale and Saturn's pole direction. Icarus 290, 14-45.

French, R. G., McGhee-French, C. A., Nicholson, P. D., Hedman, M. M. 2019. Kronoseismology III: Waves in Saturn's inner C ring. Icarus 319, 599-626.

Goldreich, P., Tremaine, S. D. 1978. The formation of the Cassini Division in Saturn's rings. Icarus 34, 240-253.

Gresh, D. L., Rosen, P. A., Tyler, G. L., Lissauer, J. J. 1986. An analysis of bending waves in Saturn's rings using Voyager radio occultation data. Icarus 68, 481-502.

Hahn, J. M. 2007. The secular evolution of a close ring-satellite system: The excitation of spiral bending waves at a nearby gap edge. The Astrophysical Journal 665, 856-865.

Hahn, J. M. 2008. The secular evolution of a close ring-satellite system: The excitation of spiral density waves at a nearby gap edge. The Astrophysical Journal 680, 1569-1581.

Hedman, M. M., Nicholson, P. D., Baines, K. H., Buratti, B. J., Sotin, C., Clark, R. N., Brown, R. H., French, R. G., Marouf, E. A. 2010. The architecture of the Cassini Division. Astron. J. 139, 228-251.

Hedman, M. M., Nicholson, P. D. 2013. Kronoseismology: Using density waves in Saturn's C ring to probe the planet's interior. Astron. J. 146, 12.

Hedman, M. M., Nicholson, P. D. 2014. More kronoseismology with Saturn's rings. Monthly Notices of the Royal Astronomical Society 444, 1369-1388.

Hedman, M. M., Nicholson, P. D. 2016. The B-ring's surface mass density from hidden density waves: Less than meets the eye? Icarus 279, 109-124.
Hedman, M. M., Nicholson, P. D., French, R. G. 2019. Kronoseismology IV: Six previously unidentified waves in Saturn's middle C ring Astron. J., 157:18.

Hedman, M. M., Nicholson, P. D. 2019. Axisymmetric density waves in Saturn's rings. Monthly Notices of the Royal Astronomical Society 485, 13-29.

Jacobson, R. A., et al. 2008. The gravity field of the Saturnian system and the orbits of the major Saturnian satellites. Paper presented at "Saturn after Cassini-Huygens" symposium, Imperial College, London.

Jerousek, R. G., Colwell, J. E., Esposito, L. W. 2011. Morphology and variability of the Titan ringlet and Huygens ringlet edges. Icarus 216, 280-291.

Lewis, M. C., Stewart, G. R. 2000. Collisional dynamics of perturbed planetary rings. I. The Astronomical Journal 120, 3295-3310.

Lehmann, M., Schmidt, J., Salo, H. 2019. Density waves and the viscous overstability in Saturn's rings.

Astronomy and Astrophysics 623, A121.

Longaretti, P.-Y., Rappaport, N. 1995. Viscous overstabilities in dense narrow planetary rings. Icarus 116, 376-396.

Marouf, E. A., Tyler, G. L. 1986.

Detection of two satellites in the Cassini division of Saturn's rings. Nature 323, 31-35.

Nicholson, P. D., Cooke, M. L., Pelton, E. 1990. An absolute radius scale for Saturn's rings. Astron. J. 100, 1339-1362.

Nicholson, P. D., French, R. G., Hedman, M. M., Marouf, E. A., Colwell, J. E. 2014a. Noncircular features in Saturn's rings: I. The edge of the $\mathrm{B}$ ring. Icarus 227, 152-175.

Nicholson, P. D., French, R. G., McGhee-French, C. A., Hedman, M. M., Marouf, E. A., Colwell, J. E., Lonergan, K., Sepersky, T. 2014b. Noncircular features in Saturn's rings: II. The C ring. Icarus 241, 373-396. 
Nicholson, P. D., De Pater, I., French, R. G., Showalter, M. R. 2018. The rings of Uranus. Planetary Ring Systems. Properties, Structure, and Evolution 93.

Nicholson, P. D., Hedman, M. M. 2016. A vertical rift in Saturn's inner C ring. Icarus 279, 78-99.

Porco, C., Danielson, G. E., Goldreich, P., Holberg, J. B., Lane, A. L. 1984.

Saturn's nonaxisymmetric ring edges at $1.95 \mathrm{R}(\mathrm{s})$ and $2.27 \mathrm{R}(\mathrm{s})$. Icarus 60 , 17-28.

Porco, C. C., and 34 colleagues 2005. Cassini Imaging Science: Initial results on Saturn's rings and small satellites. Science 307, 1226-1236.

Rappaport, N. J., Longaretti, P.-Y., French, R. G., Marouf, E. A., McGhee, C. A. 2009. A procedure to analyze nonlinear density waves in Saturn's rings using several occultation profiles. Icarus 199, 154-173.

Rappaport/Borderies, N. 1998. Ring-ringlet interactions. Icarus 132, 36-42.

Rosen, P. A., Lissauer, J. J. 1988. The Titan -1:0 nodal bending wave in Saturn's ring C. Science 241, 690-694.

Showalter, M. R., Cuzzi, J. N., Marouf, E. A., Esposito, L. W. 1986. Satellite "wakes" and the orbit of the Encke Gap moonlet. Icarus 66, 297-323.

Shu, F. H. 1984. Waves in planetary rings. In IAU Colloq. 75: Planetary Rings, Eds. A. Brahic and R. Greenberg, Univ. of Arizona Press, 513-561.

Shu, F. H., Cuzzi, J. N., Lissauer, J. J. 1983. Bending waves in Saturn's rings. Icarus 53, 185-206.

Spitale, J. N., Hahn, J. M. 2016. The shape of Saturn's Huygens ringlet viewed by Cassini ISS. Icarus 279, 141-154.
Spitale, J., Porco, C., Colwell, J. 2006. Shapes and kinematics of eccentric features in Saturn's rings from Cassini imaging and occultation observations. Bulletin of the American Astronomical Society 38, 51.05 .

Spitale, J. N., Porco, C. C. 2009. Time variability in the outer edge of Saturn's A-ring revealed by Cassini imaging. The Astronomical Journal 138, 1520-1528.

Spitale, J. N., Porco, C. C. 2010. Detection of free unstable modes and massive bodies in Saturn's outer B ring. The Astronomical Journal 140, 1747-1757.

Tajeddine, R., Nicholson, P. D., Longaretti, P.-Y., El Moutamid, M., Burns, J. A. 2017. What confines the rings of Saturn? The Astrophysical Journal Supplement Series 232, 28.

Tiscareno, M. S., Burns, J. A., Hedman, M. M., Spitale, J. N., Porco, C. C., Murray, C. D., Cassini Imaging Team 2005. Wavy edges and other disturbances in Saturn's Encke and Keeler gaps. Bulletin of the American Astronomical Society 37, 64.02.

Tiscareno, M. S., Hedman, M. M., Burns, J. A., Weiss, J. W., Porco, C. C. 2013. Probing the inner boundaries of Saturn's A ring with the Iapetus -1:0 nodal bending wave. Icarus 224, 201-208.

Tiscareno, M. S., Harris, B. E. 2018. Mapping spiral waves and other radial features in Saturn's rings. Icarus 312, 157-171.

Turtle, E., Porco, C., Haemmerle, V., Hubbard, W., Clark, R. 1991. The kinematics of eccentric features in Saturn's Cassini Division from combined Voyager and ground-based data. Bulletin of the American Astronomical Society 23, 117,9. Weiss, J. W., Porco, C. C., Tiscareno, M. S. 2009. Ring edge waves and the masses of nearby satellites. The Astronomical Journal 138, 272-286. 